\title{
Bioinspired Early Divergent Oxidative Cyclizations toward Pleiocarpamine, Talbotine, and Strictamine.
}

\author{
Maxime Jarret, ${ }^{a}$ Hussein Abou-Hamdan, ${ }^{a}$ Cyrille Kouklovsky, ${ }^{a}$ Erwan Poupon, ${ }^{b}$ Laurent \\ Evanno $^{\mathrm{b}}$ and Guillaume Vincent.*a \\ ${ }^{a}$ Institut de Chimie Moléculaire et des Matériaux d'Orsay (ICMMO), Université Paris-Saclay, CNRS \\ 91405 Orsay, France \\ ${ }^{b}$ Biomolécules: Conception, Isolement et Synthèse (BioCIS), Université Paris-Saclay, CNRS \\ 92296 Châtenay-Malabry, France \\ * guillaume.vincent@universite-paris-saclay.fr

\section{Supporting Information}

\section{Table of Content}

(I). General information

(II). Determination of the relative stereochemistry of $18 \mathrm{a}$ and $26 \mathrm{a} / \mathrm{b}$

(III). Experimental procedures and spectroscopic data of compounds

(IV). NMR spectra of compounds

S14

(V). HPLC chromatograms of $17 \mathrm{a}$ and $25 \mathrm{a}$

S40

(VI). References 


\section{General Information}

All reactions were performed using oven-dried $\left(>100^{\circ} \mathrm{C}\right.$ ) round-bottomed flasks unless otherwise stated. Where appropriate, reactions were carried out under an inert atmosphere of argon with dry solvents, unless otherwise stated. Tetrahydrofuran (THF) was distilled under argon over sodiumbenzophenone. Dichloromethane $\left(\mathrm{CH}_{2} \mathrm{Cl}_{2}\right)$, was distilled under argon over $\mathrm{CaH}_{2}$. Other reagent-grade chemicals and solvents were obtained from commercial suppliers and were used as received. Reactions were monitored with analytical thin-layer chromatography (TLC) on silica gel 60 F254 plates and visualized under UV ( $254 \mathrm{~nm}$ ) and/or by staining with phosphomolybdic acid hydrate solution (6\%) in EtOH followed by heating. ${ }^{1} \mathrm{H}$ NMR spectra were recorded on Bruker DRX300 (300 MHz), Bruker AM360 (360 MHz) and Bruker $400(400 \mathrm{MHz})$ instruments at $295 \mathrm{~K}$; chemical shifts $(\delta)$ are given in parts per million with respect to the residual protonated solvent $(\delta=7.26 \mathrm{ppm}$ for chloroform- $d$ ), which served as an internal standard. ${ }^{13} \mathrm{C}$ NMR spectra were recorded on Bruker DRX300 $(300 \mathrm{MHz})$, Bruker AM360 (360 MHz) and Bruker $400(400 \mathrm{MHz})$ instruments, at $295 \mathrm{~K}$; chemical shifts are expressed with respect to the deuterated solvent $(\delta=77.16 \mathrm{ppm}$ for chloroform- $d$ ). Coupling constant(s) in hertz $(\mathrm{Hz})$ were measured from one-dimensional spectra and multiplicities were abbreviated as following: br (broad), $s$ (singlet), $d$ (doublet), $t$ (triplet), q (quadruplet), $m$ (multiplet). Infrared spectra were recorded as thin films on $\mathrm{NaCl}$ plates using PerkinElmer Spectrum One instrument. High resolution mass spectra (HRMS) were recorded using Electrospray Ionization (ESI) method with a Bruker Daltonics MicrOTOF-Q instrument. $[\alpha]_{D}$ were measured on Anton Paar MCP150 polarimeter. Chiral HPLC were performed on Dionex UltiMate 3000 instrument. 


\section{Determination of the relative stereochemistry of $18 a$ and $26 a / b$}

The determination of the relative stereochemistry of diastereoisomers (+)-18a and (-)-26a was performed on their respectively cyclized products compound (+)-18a and (-)-26a via NOESY experiments. The cis relationship between hydrogens $\mathrm{H} 3$ and $\mathrm{H} 15$ of $(+)-18 \mathrm{a}$ was demonstrated by a NOESY correlation between $\mathrm{H} 3$ and $\mathrm{H} 15$ as well as the correlation of $\mathrm{H} 3$ and $\mathrm{H} 15$ with the same hydrogen at position 14 . The fact that for diasteroisomer (-)-26a and $\mathbf{2 6 b}, \mathrm{H3}$ and one of the hydrogen at position 20 are correlated together proved the trans relationship between protons $\mathrm{H} 3$ and $\mathrm{H} 15$. See copies of the NOESY 2D spectra in part IV.
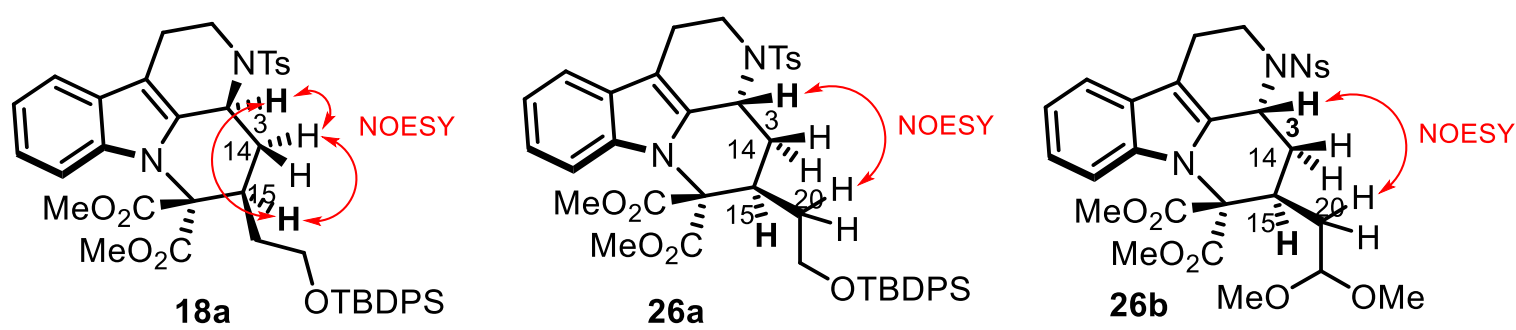

Figure S1. Determination of the relative stereochemistry of (+)-18a, (-)-26a and $\mathbf{2 6 b}$. 


\section{Experimental procedures and spectroscopic data of compounds}

Compound S1. Prepared according to literature procedure. ${ }^{1}$

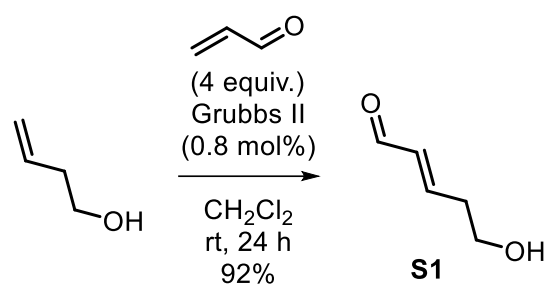

To a solution of 3-buten-1-ol (1.0 mL, $11.6 \mathrm{mmol})$ and acrolein $(3.1 \mathrm{~mL}, 46.5 \mathrm{mmol})$ in $\mathrm{CH}_{2} \mathrm{Cl}_{2}(4.2 \mathrm{~mL})$ at $\mathrm{rt}$ was added $2^{\text {nd }}$ generation Grubbs catalyst $(77 \mathrm{mg}, 90.7 \mu \mathrm{mol})$ solid in one portion. After $24 \mathrm{~h}$ at $\mathrm{rt}$, the reaction mixture was directly submitted to column chromatography $\left(\mathrm{Et}_{2} \mathrm{O} 100 \%\right)$ to yield $\mathbf{S 1}$ $(1.07 \mathrm{~g}, 10.7 \mathrm{mmol}, 92 \%)$ as a yellow oil.

Spectral data were consistent with those reported. ${ }^{1}$

${ }^{*}{ }^{1}$ H NMR (300 MHz, chloroform-d): $\delta 9.52$ (d, $J=7.9 \mathrm{~Hz}, 1 \mathrm{H}$ ), 6.90 (dt, $J=15.7,6.9 \mathrm{~Hz}, 1 \mathrm{H}$ ), 6.20 (ddt, $J=15.7,7.9,1.4 \mathrm{~Hz}, 1 \mathrm{H}), 3.86-3.82(\mathrm{~m}, 2 \mathrm{H}), 2.60(\mathrm{qd}, J=6.5,1.4 \mathrm{~Hz}, 2 \mathrm{H}), 1.82(\mathrm{bs}, 1 \mathrm{H})$.

Compound 21a. Prepared using slightly modified literature procedure. ${ }^{2}$

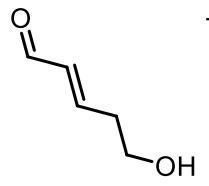

s1

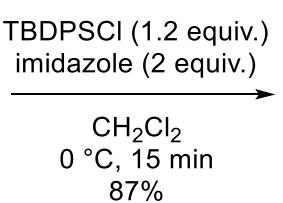

$87 \%$

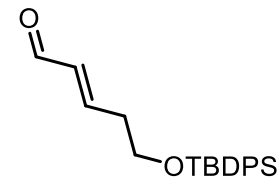

21a

To a solution of $\mathbf{S 1}(500 \mathrm{mg}, 4.99 \mathrm{mmol})$ in $\mathrm{CH}_{2} \mathrm{Cl}_{2}(17 \mathrm{~mL})$ at $0{ }^{\circ} \mathrm{C}$ was added sequentially imidazole (680 mg, $9.99 \mathrm{mmol})$ in one portion and TBDPSCl $(1.56 \mathrm{~mL}, 5.99 \mathrm{mmol})$ dropwise. After $15 \mathrm{~min}$ at $0{ }^{\circ} \mathrm{C}$, the reaction mixture was quenched with $\mathrm{H}_{2} \mathrm{O}(20 \mathrm{~mL})$. After partitioning, the aqueous layer was extracted with $\mathrm{CH}_{2} \mathrm{Cl}_{2}(2 \times 15 \mathrm{~mL})$. The combined organic layers were washed with $\mathrm{H}_{2} \mathrm{O}(20 \mathrm{~mL})$ and sat brine $(20 \mathrm{~mL})$, dried over $\mathrm{Na}_{2} \mathrm{SO}_{4}$ and concentrated in vacuo. The crude residue was purified two times by column chromatography (petroleum ether/ $/ \mathrm{Et}_{2} \mathrm{O} 96: 4$ to $94: 6$ ) to yield $21 \mathrm{a}$ ( $1.47 \mathrm{~g}, 4.35 \mathrm{mmol}, 87 \%$ ) as a colorless oil.

Spectral data were consistent with those reported. ${ }^{2}$

${ }^{*}{ }^{1} \mathrm{H}$ NMR $(360 \mathrm{MHz}$, chloroform- $d): \delta 9.48(\mathrm{~d}, J=7.8 \mathrm{~Hz}, 1 \mathrm{H}), 7.66-7.64(\mathrm{~m}, 4 \mathrm{H}), 7.47-7.37(\mathrm{~m}, 6 \mathrm{H}), 6.84$ (dt, $J=15.6,7.0 \mathrm{~Hz}, 1 \mathrm{H}$ ), $6.15(\mathrm{ddt}, J=15.6,7.8,1.4 \mathrm{~Hz}, 1 \mathrm{H}), 3.83(\mathrm{t}, J=6.1 \mathrm{~Hz}, 2 \mathrm{H}), 2.55$ (qd, $J=6.5$, $1.4 \mathrm{~Hz}, 2 \mathrm{H}), 1.05(\mathrm{~s}, 9 \mathrm{H})$.

Compound 21b. Prepared using slightly modified literature procedure. ${ }^{3}$

\footnotetext{
${ }^{1}$ Zhang, W.; Bah, J.; Wohlfarth, A.; Franzén, J., Chem. - Eur. J. 2011, 17, 13814-13824.

${ }^{2}$ Zhang, H.; Ma, X.; Kang, H.; Hong, L.; Wang, R., Chem. - Asian J. 2013, 8, 542-545.

${ }^{3}$ Rodrigo, E.; García Ruano, J. L.; Cid, M. B., J. Org. Chem., 2013, 78, 10737-10746.
} 


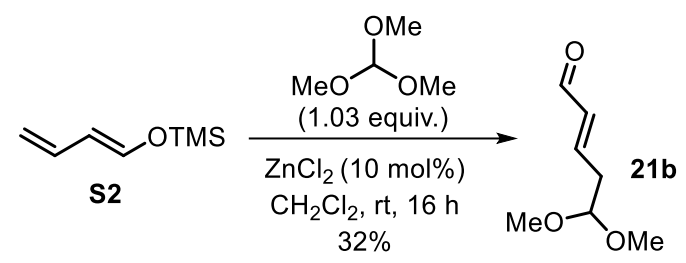

$\mathrm{ZnCl}_{2}$ (479 mg, $3.51 \mathrm{mmol}$ ) was added to a mixture of 1-trimethylsilyloxy-1,3-butadiene $\mathbf{S 2 ~ ( 5 ~ g , ~} 35.14$ $\mathrm{mmol}$ ) and trimethyl orthoformate $(3.96 \mathrm{~mL}, 36.20 \mathrm{mmol})$ in dry $\mathrm{CH}_{2} \mathrm{Cl}_{2}(120 \mathrm{~mL})$. The mixture was stirred at room temperature overnight whereupon it was poured into water $(60 \mathrm{~mL})$. The aqueous layer was extracted with $\mathrm{CH}_{2} \mathrm{Cl}_{2}(2 \times 100 \mathrm{~mL})$, and combined organic layers were washed with brine (60 $\mathrm{mL}$ ), dried over $\mathrm{Na}_{2} \mathrm{SO}_{4}$, filtered, concentrated, and purified by column chromatography on silica gel using petroleum ether/EtOAc (9/1) as an eluent to give aldehyde $\mathbf{2 1 b}(1.64 \mathrm{~g}, 11.37 \mathrm{mmol}, 32 \%)$ as a yellow oil.

Spectral data were consistent with those reported. ${ }^{3}$

* Rf: $0.42 \mathrm{PE} / \mathrm{EtOAC}(7 / 3)$.

* ${ }^{1} \mathrm{H}$ NMR $(300 \mathrm{MHz}$, chloroform-d) $\delta 9.50(\mathrm{~d}, J=7.9 \mathrm{~Hz}, 1 \mathrm{H}), 6.79(\mathrm{dt}, J=15.8,7.2 \mathrm{~Hz}, 1 \mathrm{H}), 6.17$ (ddt, $J$ $=15.8,7.9,1.5 \mathrm{~Hz}, 1 \mathrm{H}), 4.50(\mathrm{t}, J=5.7 \mathrm{~Hz}, 1 \mathrm{H}), 3.34(\mathrm{~s}, 6 \mathrm{H}), 2.66-2.62(\mathrm{~m}, 2 \mathrm{H})$.

${ }^{*}{ }^{13}$ C NMR (75 MHz, chloroform-d) $\delta 193.9,152.4,135.1,102.7,53.4$ (2C), 36.4.

Compound 23a. Prepared using slightly modified literature procedure. ${ }^{2}$

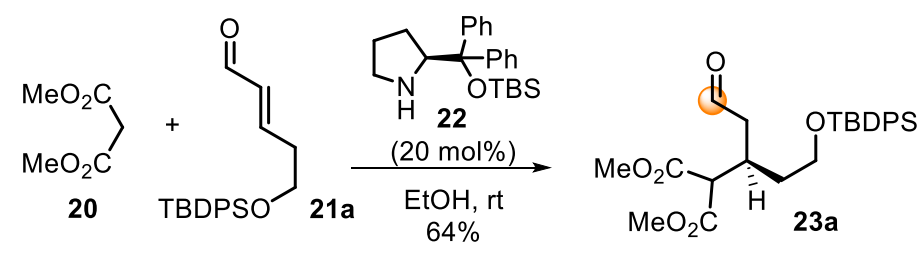

To a solution of $21 \mathrm{a}(300 \mathrm{mg}, 0.886 \mathrm{mmol})$ in absolute EtOH $(1.8 \mathrm{~mL})$ at $\mathrm{rt}$ was added successively (-)$22(65 \mathrm{mg}, 0.177 \mathrm{mmol}$ ) and dimethyl malonate 20 (304 $\mu \mathrm{L}, 2.66 \mathrm{mmol})$. After $24 \mathrm{~h}$ at rt, the reaction mixture was concentrated in vacuo. The crude residue was purified by column chromatography (petroleum ether/EtOAc 94:6 to $86: 14$ ) to yield 23a ( $265 \mathrm{mg}, 0.563 \mathrm{mmol}, 64 \%$ ) as a colorless oil.

Spectral data were consistent with those reported. ${ }^{2}$

* Rf: 0.43 (petroleum ether/EtOAc 85:15).

* ee: was determined at the next step.

* IR (neat): $U_{\max }\left(\mathrm{cm}^{-1}\right)=2954,2857,1732,1429,1159,1111,738,703$.

* HRMS $\left(\mathrm{ESI}^{+}\right) \mathrm{m} / \mathrm{z}$ : calculated for $\mathrm{C}_{26} \mathrm{H}_{34} \mathrm{O}_{6} \mathrm{SiNa}^{+}[\mathrm{M}+\mathrm{Na}]^{+}$493.2017, found 493.2012.

${ }^{*}{ }^{1} \mathrm{H}$ NMR (360 MHz, chloroform-d): $\delta 9.70(\mathrm{~s}, 1 \mathrm{H}), 7.66(\mathrm{~d}, J=7.0 \mathrm{~Hz}, 4 \mathrm{H}), 7.46-7.37(\mathrm{~m}, 6 \mathrm{H}), 3.73-3.70$ $(\mathrm{m}, 3 \mathrm{H}), 3.72(\mathrm{~s}, 3 \mathrm{H}), 3.71(\mathrm{~s}, 3 \mathrm{H}), 3.01(\mathrm{sext}, J=6.5 \mathrm{~Hz}, 1 \mathrm{H}), 2.75(\mathrm{ddd}, J=18.1,5.6,1.0 \mathrm{~Hz}, 1 \mathrm{H}), 2.60$ (ddd, $J=18.1,6.5,1.5 \mathrm{~Hz}, 1 \mathrm{H}), 1.79-1.61(\mathrm{~m}, 2 \mathrm{H}), 1.06(\mathrm{~s}, 9 \mathrm{H})$.

${ }^{*}{ }^{13}$ C NMR (91 MHz, chloroform-d): $\delta$ 201.1, 169.2, 168.9, 135.6 (4C), 133.5 (2C), $129.8(2 \mathrm{C}), 127.8(4 \mathrm{C})$, $61.7,53.9,52.5,52.4,45.7,34.5,30.1,26.9$ (3C), 19.2. 


\section{Compound 23b.}

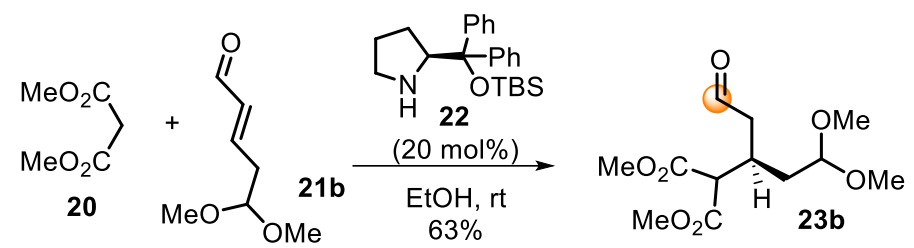

To a solution of $21 \mathrm{~b}(200 \mathrm{mg}, 1.39 \mathrm{mmol})$ in absolute EtOH $(2.8 \mathrm{~mL})$ at $\mathrm{rt}$ was added successively $(-)$ $22(102 \mathrm{mg}, 0.28 \mathrm{mmol})$ and dimethyl malonate $20(478 \mu \mathrm{L}, 4.16 \mathrm{mmol})$. After $40 \mathrm{~h}$ at rt, the reaction mixture was concentrated in vacuo. The crude residue was purified by column chromatography $\left(\mathrm{CH}_{2} \mathrm{Cl}_{2} / \mathrm{EtOAc} 9: 1\right)$ to yield $\mathbf{2 3 b}$ ( $\left.242 \mathrm{mg}, 0.876 \mathrm{mmol}, 63 \%\right)$ as a yellow oil.

* Rf: $0.29 \mathrm{CH}_{2} \mathrm{Cl}_{2} /$ EtOAc (95/5).

* ee: Not determined (not stable)

* HRMS $\left(\mathrm{ESI}^{+}\right) \mathrm{m} / \mathrm{z}$ : calculated for $\mathrm{C}_{12} \mathrm{H}_{20} \mathrm{NaO}_{7}^{+}[\mathrm{M}+\mathrm{Na}]^{+}$299.1101, found 299.1095.

* ${ }^{1} \mathrm{H}$ NMR $\left(360 \mathrm{MHz}, \mathrm{CDCl}_{3}\right) \delta 9.68(\mathrm{~s}, 1 \mathrm{H}), 4.38(\mathrm{t}, J=5.4 \mathrm{~Hz}, 1 \mathrm{H}), 3.71(\mathrm{~s}, 3 \mathrm{H}), 3.70(\mathrm{~s}, 3 \mathrm{H}), 3.64(\mathrm{~d}, J=$ $5.9 \mathrm{~Hz}, 1 \mathrm{H}), 3.27(\mathrm{~s}, 6 \mathrm{H}), 2.90-2.81(\mathrm{~m}, 1 \mathrm{H}), 2.74-2.60(\mathrm{~m}, 2 \mathrm{H}), 1.79-1.62(\mathrm{~m}, 2 \mathrm{H})$.

${ }^{*}{ }^{13} \mathrm{C}$ NMR $\left(75 \mathrm{MHz}, \mathrm{CDCl}_{3}\right) \delta 200.9,169.1,168.9,103.2,54.2,53.4,52.9,52.6,52.5,46.0,34.9,29.0$.

\section{Compound (+)-17a and (-)-25a.}<smiles>CCOCC(CC=O)C(CC=O)C(OC)OC</smiles>
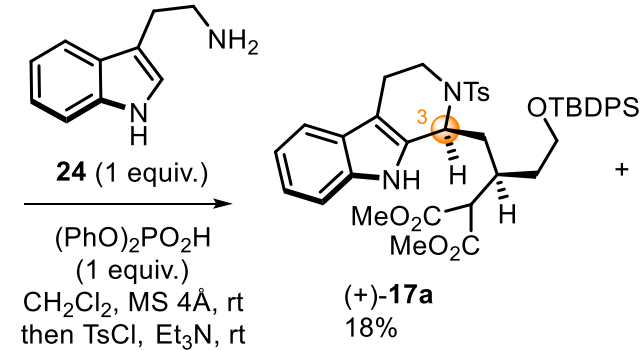

$(+)-17 \mathrm{a}$

$18 \%$

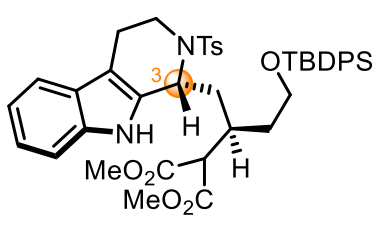

$(-)-25 a$

$21 \%$

A solution of tryptamine $24(80 \mathrm{mg}, 0.499 \mathrm{mmol}), 23 \mathrm{a}(235 \mathrm{mg}, 0.499 \mathrm{mmol}),(\mathrm{PhO})_{2} \mathrm{PO}_{2} \mathrm{H}(125 \mathrm{mg}$, $0.499 \mathrm{mmol})$, MS $4 \AA$ ( $0.75 \mathrm{~g})$ in $\mathrm{CH}_{2} \mathrm{Cl}_{2}(10 \mathrm{~mL})$ was stirred at $\mathrm{rt}$ for $24 \mathrm{~h}$. Et $\mathrm{E}_{3} \mathrm{~N}(348 \mu \mathrm{L}, 2.50 \mathrm{mmol})$ was added dropwise followed by $\mathrm{TsCl}(144 \mathrm{mg}, 0.749 \mathrm{mmol}$ ) in one portion. After $16 \mathrm{~h}$ at rt, the reaction mixture was filtered on celite $\left(\mathrm{CH}_{2} \mathrm{Cl}_{2}\right.$ rinse). The reaction mixture was quenched with sat aq $\mathrm{NH}_{4} \mathrm{Cl}(10$ $\mathrm{mL})$. The aqueous layer was extracted with $\mathrm{CH}_{2} \mathrm{Cl}_{2}(2 \times 10 \mathrm{~mL})$. The combined organic layers were dried over $\mathrm{Na}_{2} \mathrm{SO}_{4}$ and concentrated in vacuo. The crude residue was purified by column chromatography (petroleum ether/EtOAc 9:1 to 3:1) to yield (+)-17a (70 mg, $91.3 \mu \mathrm{mol}, 18 \%$ ) as a light-yellow foam and (-)-25a (79 mg, $0.103 \mathrm{mmol}, 21 \%)$ as a light-yellow foam.

\section{Data for $(+)-17 a$.}

* Rf: 0.24 (petroleum ether/ $\mathrm{Et}_{2} \mathrm{O} 3: 2$ ).

$*[\boldsymbol{\alpha}]_{\mathrm{D}}^{20}:+22.0\left(c=1.00, \mathrm{CH}_{2} \mathrm{Cl}_{2}\right)$.

* IR (neat): $U_{\max }\left(\mathrm{cm}^{-1}\right)=3390,2953,2857,1731,1428,1336,1196,1161,1111,1092,822,739,708$.

* HRMS $\left(E S I^{+}\right) \mathrm{m} / z$ : calculated for $\mathrm{C}_{43} \mathrm{H}_{50} \mathrm{~N}_{2} \mathrm{O}_{7} \mathrm{SSiNa}^{+}[\mathrm{M}+\mathrm{Na}]^{+}$789.3000, found 789.2991. 
* ${ }^{1} \mathrm{H}$ NMR (360 MHz, chloroform-d): $\delta 8.16$ (bs, $\left.1 \mathrm{H}\right), 7.69-7.65(\mathrm{~m}, 4 \mathrm{H}), 7.59(\mathrm{~d}, J=8.1 \mathrm{~Hz}, 2 \mathrm{H}), 7.41-$ $7.32(\mathrm{~m}, 6 \mathrm{H}), 7.26(\mathrm{~d}, J=7.7 \mathrm{~Hz}, 1 \mathrm{H}), 7.22(\mathrm{~d}, J=7.7 \mathrm{~Hz}, 1 \mathrm{H}), 7.12(\mathrm{t}, J=7.7 \mathrm{~Hz}, 1 \mathrm{H}), 7.04-7.00(\mathrm{~m}, 3 \mathrm{H})$, $5.17(\mathrm{t}, J=6.5 \mathrm{~Hz}, 1 \mathrm{H}), 4.04-3.99(\mathrm{~m}, 2 \mathrm{H}), 3.87-3.66(\mathrm{~m}, 2 \mathrm{H}), 3.78(\mathrm{~s}, 3 \mathrm{H}), 3.72(\mathrm{~s}, 3 \mathrm{H}), 3.23(\mathrm{ddd}, J=$ 15.0, 11.5, $5.0 \mathrm{~Hz}, 1 \mathrm{H}$ ), 2.75 (sext, $J=5.9 \mathrm{~Hz}, 1 \mathrm{H}$ ), 2.38 (dd, J=15.5, $4.6 \mathrm{~Hz}, 1 \mathrm{H}$ ), 2.31 (dd, $J=11.5,5.4$ $\mathrm{Hz}, 1 \mathrm{H}), 2.23(\mathrm{~s}, 3 \mathrm{H}), 1.98(\mathrm{t}, J=6.5 \mathrm{~Hz}, 2 \mathrm{H}), 1.94-1.91(\mathrm{~m}, 1 \mathrm{H}), 1.73-1.63(\mathrm{~m}, 1 \mathrm{H}), 1.06(\mathrm{~s}, 9 \mathrm{H})$.

${ }^{*}{ }^{13}$ C NMR (63 MHz, chloroform- $d$ ): $\delta 170.3,169.3,143.3,138.1,136.0,135.7$ (4C), 133.9, 133.7, 133.1, $129.8(2 \mathrm{C}), 129.5$ (2C), 127.9 (2C), 127.8 (2C), 126.9 (2C), 126.7, 122.0, 119.4, 118.2, 111.1, 107.5, 62.6, $53.6,52.6,52.5,51.8,39.4,38.2,35.2,32.9,27.1$ (3C), 21.5, 19.6, 19.4.

* HPLC (ChiralPak AD-H column at $25^{\circ} \mathrm{C}$, Hexane/EtOH: 95/5, $1 \mathrm{~mL} / \mathrm{min}$ ): ee $>93 \%$, t(minor) = $9.1 \mathrm{~min}$; $\mathrm{t}($ major $)=11.9 \mathrm{~min}$.

\section{Data for (-)-25a.}

* Rf: 0.41 (petroleum ether/ $\mathrm{Et}_{2} \mathrm{O} 3: 2$ ).

$*[\alpha]_{\mathrm{D}}^{20}:-28.0\left(c=1.00, \mathrm{CH}_{2} \mathrm{Cl}_{2}\right)$.

* IR (neat): $U_{\max }\left(\mathrm{cm}^{-1}\right)=3392,2953,2857,1745,1429,1333,1160,1112,913,738,702$.

* HRMS $\left(\mathrm{ESI}^{+}\right) \mathrm{m} / \mathrm{z}$ : calculated for $\mathrm{C}_{43} \mathrm{H}_{50} \mathrm{~N}_{2} \mathrm{O}_{7} \mathrm{SSiNa}^{+}[\mathrm{M}+\mathrm{Na}]^{+}$789.3000, found 789.3004.

$*{ }^{1} \mathrm{H}$ NMR $(300 \mathrm{MHz}$, chloroform-d): $\delta 7.84$ (bs, $1 \mathrm{H}), 7.69$ (dd, $\left.J=7.7,1.5 \mathrm{~Hz}, 4 \mathrm{H}\right), 7.56(\mathrm{~d}, J=8.3 \mathrm{~Hz}$, $2 \mathrm{H}), 7.45-7.36(\mathrm{~m}, 6 \mathrm{H}), 7.22(\mathrm{~d}, J=7.7 \mathrm{~Hz}, 1 \mathrm{H}), 7.17(\mathrm{~d}, J=7.7 \mathrm{~Hz}, 1 \mathrm{H}), 7.10(\mathrm{td}, J=7.7,1.0 \mathrm{~Hz}, 1 \mathrm{H})$, 7.02-6.97 (m, 3H), $5.14(\mathrm{dd}, J=9.6,4.5 \mathrm{~Hz}, 1 \mathrm{H}), 4.07(\mathrm{dd}, J=14.9,5.6 \mathrm{~Hz}, 1 \mathrm{H}), 3.91(\mathrm{~d}, J=5.5 \mathrm{~Hz}, 1 \mathrm{H})$, $3.85(\mathrm{t}, J=6.1 \mathrm{~Hz}, 2 \mathrm{H}), 3.75(\mathrm{~s}, 3 \mathrm{H}), 3.72(\mathrm{~s}, 3 \mathrm{H}), 3.39$ (ddd, $J=14.8,12.0,5.0 \mathrm{~Hz}, 1 \mathrm{H}), 2.82-2.72(\mathrm{~m}, 1 \mathrm{H})$, 2.41-2.24 (m, 2H), $2.21(\mathrm{~s}, 3 \mathrm{H}), 2.10$ (ddd, $J=14.8,9.6,4.2 \mathrm{~Hz}, 1 \mathrm{H}), 1.98-1.89(\mathrm{~m}, 3 \mathrm{H}), 1.05(\mathrm{~s}, 9 \mathrm{H})$.

${ }^{*}{ }^{13} \mathrm{C}$ NMR (63 MHz, chloroform-d): $\delta$ 169.9, 169.7, 143.3, 138.0, 135.9, 135.7 (4C), 133.8 (2C), 132.8, 129.9 (2C), 129.4 (2C), 127.9 (4C), 126.9 (2C), 126.8, 122.0, 119.4, 118.1, 111.0, 107.7, 62.4, 54.4, 52.6, $52.5,51.5,39.1,37.5,34.2,33.4,27.0(3 C), 21.5,19.4,19.4$.

* HPLC (ChiralPak AD-H column at $5^{\circ} \mathrm{C}$, Hexane/EtOH: 95/5, $1 \mathrm{~mL} / \mathrm{min}$ ): ee $>93 \%$, $\mathrm{t}($ minor) $=8.7 \mathrm{~min}$; $\mathrm{t}($ major $)=9.9 \mathrm{~min}$.

\section{Compound $17 \mathrm{~b}$ and $25 \mathrm{~b}$.}
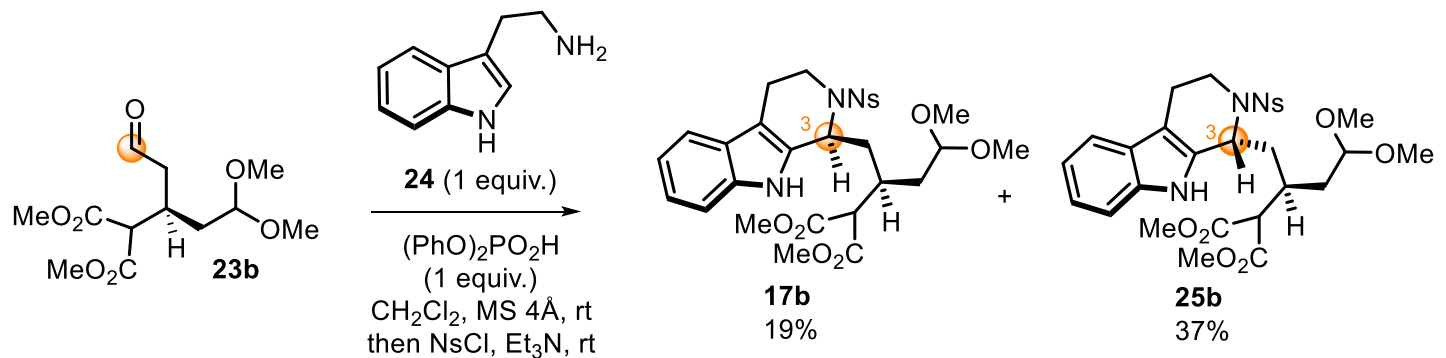

A solution of aldehyde $23 \mathrm{~b}(96 \mathrm{mg}, 0.347 \mathrm{mmol})$, tryptamine $24\left(61 \mathrm{mg}, 0.382 \mathrm{mmol},(\mathrm{PhO})_{2} \mathrm{PO}_{2} \mathrm{H}(87\right.$ $\mathrm{mg}, 0.347 \mathrm{mmol}), \mathrm{MS} 4 \AA$ ( $520 \mathrm{mg})$ in $\mathrm{CH}_{2} \mathrm{Cl}_{2}(7 \mathrm{~mL})$ was stirred at rt for $24 \mathrm{~h}$. Et $3 \mathrm{~N}(96.7 \mu \mathrm{L}, 0.694 \mathrm{mmol})$ was added dropwise followed by $\mathrm{NsCl}(154 \mathrm{mg}, 0.694 \mathrm{mmol}$ ) in one portion. After $16 \mathrm{~h}$ at $\mathrm{rt}$, the reaction mixture was filtered on celite $\left(\mathrm{CH}_{2} \mathrm{Cl}_{2}\right.$ rinse). The reaction mixture was quenched with water. 
The aqueous layer was extracted with $\mathrm{CH}_{2} \mathrm{Cl}_{2}(2 \times 10 \mathrm{~mL})$. The combined organic layers were dried over $\mathrm{Na}_{2} \mathrm{SO}_{4}$ and concentrated in vacuo. The crude residue was purified by column chromatography $\left(\mathrm{CH}_{2} \mathrm{Cl}_{2} / \mathrm{EtOAc} 100: 0\right.$ to $\left.97: 3+0.1 \% \mathrm{Et}_{3} \mathrm{~N}\right)$ to yield $17 \mathrm{~b}(40 \mathrm{mg}, 66.3 \mu \mathrm{mol}, 19 \%)$ as a yellow foam and 25b (77 mg, $0.127 \mathrm{mmol}, 37 \%$ ) as a yellow foam.

\section{Data for 17b.}

* Rf: $0.39 \mathrm{CH}_{2} \mathrm{Cl}_{2} / \mathrm{EtOAC}(97 / 3)$.

* HRMS (ESI $\left.{ }^{+}\right) \mathrm{m} / \mathrm{z}$ : calculated for $\mathrm{C}_{28} \mathrm{H}_{34} \mathrm{~N}_{3} \mathrm{O}_{10} \mathrm{~S}^{+}[\mathrm{M}+\mathrm{H}]^{+}$604.1959, found 604.1934. calculated for $\mathrm{C}_{28} \mathrm{H}_{33} \mathrm{~N}_{3} \mathrm{NaO}_{10} \mathrm{~S}^{+}[\mathrm{M}+\mathrm{Na}]^{+} 626.1779$, found 626.1759 .

* ${ }^{1} \mathrm{H}$ NMR (300 MHz, chloroform-d) $\delta 8.64$ (brs, $\left.1 \mathrm{H}\right), 8.11(\mathrm{~d}, J=8.8 \mathrm{~Hz}, 2 \mathrm{H}), 7.89(\mathrm{~d}, J=8.8 \mathrm{~Hz}, 2 \mathrm{H})$, 7.31-7.23 (m, 2H), $7.15(\mathrm{td}, J=7.5,1.0 \mathrm{~Hz}, 1 \mathrm{H}), 7.02(\mathrm{td}, J=7.5,1.0 \mathrm{~Hz}, 1 \mathrm{H}), 5.24(\mathrm{t}, J=7.2 \mathrm{~Hz}, 1 \mathrm{H}), 4.53$ $(\mathrm{t}, J=4.9 \mathrm{~Hz}, 1 \mathrm{H}), 4.20(\mathrm{dd}, J=15.1,6.0 \mathrm{~Hz}, 1 \mathrm{H}), 3.81(\mathrm{~d}, J=4.5 \mathrm{~Hz}, 1 \mathrm{H}), 3.75(\mathrm{~s}, 3 \mathrm{H}), 3.74(\mathrm{~s}, 3 \mathrm{H}), 3.56-$ $3.49(\mathrm{~m}, 1 \mathrm{H}), 3.47(\mathrm{~s}, 3 \mathrm{H}), 3.43(\mathrm{~s}, 3 \mathrm{H}), 2.70-2.62(\mathrm{~m}, 1 \mathrm{H}), 2.56(\mathrm{dd}, J=16.2,4.5 \mathrm{~Hz}, 1 \mathrm{H}), 2.35(\mathrm{~m}, 1 \mathrm{H})$, $2.07(\mathrm{t}, J=6.8 \mathrm{~Hz}, 2 \mathrm{H}), 2.02(\mathrm{t}, J=5.7 \mathrm{~Hz}, 1 \mathrm{H}), 1.81(\mathrm{dt}, J=14.7,6.4 \mathrm{~Hz}, 1 \mathrm{H})$

* ${ }^{13}$ C NMR (75 MHz, chloroform-d $\delta$ 169.6, 169.0, 149.8, 146.8, 135.9, 132.1, 128.0 (2C), 126.3, 124.2 (2C), 122.5, 119.7, 118.2, 111.1, 107.1, 104.4, 54.8, 54.4, 53.3, 52.7, 52.7, 52.1, 39.7, 38.3, 34.8, 31.2, 19.9 .

\section{Data for 25b.}

* Rf: $0.23 \mathrm{CH}_{2} \mathrm{Cl}_{2} / \mathrm{EtOAC}(97 / 3)$.

* HRMS (ESI $\left.{ }^{+}\right) \mathrm{m} / z$ : calculated for $\mathrm{C}_{28} \mathrm{H}_{34} \mathrm{~N}_{3} \mathrm{O}_{10} \mathrm{~S}^{+}[\mathrm{M}+\mathrm{H}]^{+}$604.1959, found 604.1929. calculated for $\mathrm{C}_{28} \mathrm{H}_{33} \mathrm{~N}_{3} \mathrm{NaO}_{10} \mathrm{~S}^{+}[\mathrm{M}+\mathrm{Na}]^{+} 626.1779$, found 626.1752 .

* ${ }^{1} \mathrm{H}$ NMR $(300 \mathrm{MHz}$, chloroform- $d$ ) $\delta 8.45$ (brs, $1 \mathrm{H}), 8.01(\mathrm{~d}, J=8.7 \mathrm{~Hz}, 2 \mathrm{H}), 7.86(\mathrm{~d}, J=8.7 \mathrm{~Hz}, 2 \mathrm{H})$, $7.27(\mathrm{~m}, 1 \mathrm{H}), 7.18(\mathrm{~d}, J=7.5 \mathrm{~Hz}, 1 \mathrm{H}), 7.12(\mathrm{t}, J=7.5 \mathrm{~Hz}, 1 \mathrm{H}), 6.99(\mathrm{t}, J=7.5 \mathrm{~Hz}, 1 \mathrm{H}), 5.25(\mathrm{dd}, J=10.2$, $3.4 \mathrm{~Hz}, 1 \mathrm{H}), 4.63(\mathrm{t}, J=5.3 \mathrm{~Hz}, 1 \mathrm{H}), 4.13(\mathrm{dd}, J=15.1,6.0 \mathrm{~Hz}, 1 \mathrm{H}), 3.95(\mathrm{~d}, J=4.5 \mathrm{~Hz}, 1 \mathrm{H}), 3.79(\mathrm{~s}, 3 \mathrm{H})$, $3.74(\mathrm{~s}, 3 \mathrm{H}), 3.55-3.48(\mathrm{~m}, 1 \mathrm{H}), 3.44(\mathrm{~s}, 3 \mathrm{H}), 3.42(\mathrm{~s}, 3 \mathrm{H}), 2.77-2.64(\mathrm{~m}, 1 \mathrm{H}), 2.41(\mathrm{dd}, J=15.8,4.5 \mathrm{~Hz}$, $1 \mathrm{H}), 2.28-2.03(\mathrm{~m}, 3 \mathrm{H}), 2.03-1.89(\mathrm{~m}, 2 \mathrm{H})$.

* ${ }^{13}$ C NMR (90 MHz, chloroform-d) $\delta 169.8,169.5,149.7,146.6,135.9,132.3,128.0$ (2C), 126.3, 123.9 (2C), 122.4, 119.6, 118.1, 111.0, 107.0, 104.5, 54.1, 54.0, 53.7, 52.7, 52.7, 52.0, 39.2, 37.4, 34.6, 31.9, 19.4 .

\section{Compounds (+)-18a and (-)-19a.}

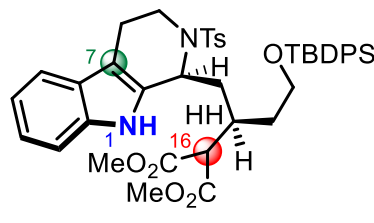

$(+)-17 a$

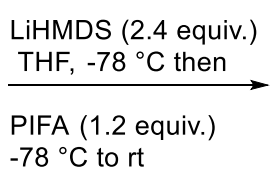

$-78^{\circ} \mathrm{C}$ to rt

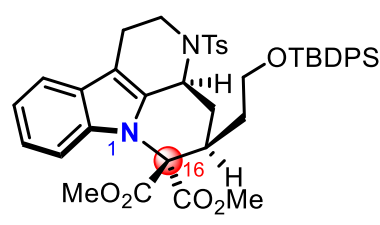

$(+)-18 a$

To a solution of (+)-cis-17a $(136 \mathrm{mg}, 0.177 \mathrm{mmol})$ in THF $(2.7 \mathrm{~mL})$ at $-78^{\circ} \mathrm{C}$ was added LiHMDS $(461 \mu \mathrm{L}$, 1.0 $\mathrm{M}$ in THF, $0.461 \mathrm{mmol}$ ) dropwise. After $30 \mathrm{~min}$ at $-78^{\circ} \mathrm{C}$, a solution of PIFA (92 $\mathrm{mg}, 0.213 \mathrm{mmol}$ ) in 
THF $(750 \mu \mathrm{L})$ was added dropwise. The reaction mixture was allowed to warm to rt, stirred for $30 \mathrm{~min}$ and quenched with $\mathrm{H}_{2} \mathrm{O}(5 \mathrm{~mL})$. The aqueous layer was diluted with sat brine $(5 \mathrm{~mL})$ and extracted with EtOAc $(3 \times 10 \mathrm{~mL})$. The combined organic layers were washed with sat brine, dried over $\mathrm{Na}_{2} \mathrm{SO}_{4}$ and concentrated in vacuo. The crude residue was purified by column chromatography (petroleum ether/EtOAc $95: 5$ to $8: 2$ ) to yield (+)-18a (31 mg, $40.5 \mu \mathrm{mol}, 23 \%$ ) as a white foam.

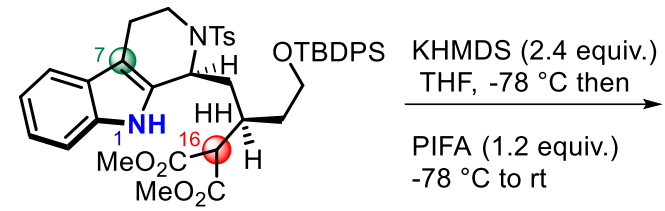

(+)-17a

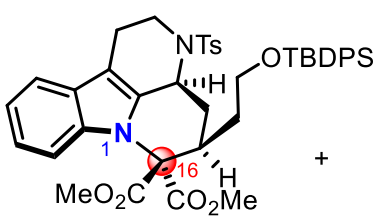

(+)-18a

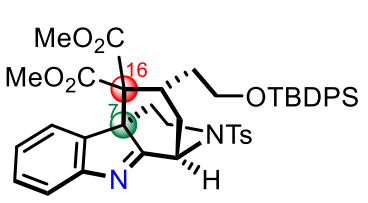

(-)-19a

To a solution of (+)-17a (145 mg, $0.189 \mathrm{mmol})$ in THF ( $2.9 \mathrm{~mL})$ at $-78^{\circ} \mathrm{C}$ was added KHMDS (908 $\mu \mathrm{L}, 0.5$ $\mathrm{M}$ in PhMe, $0.454 \mathrm{mmol}$ ) dropwise. After $30 \mathrm{~min}$ at $-78{ }^{\circ} \mathrm{C}$, a solution of PIFA (98 $\mathrm{mg}, 0.227 \mathrm{mmol}$ ) in THF $(0.7 \mathrm{~mL}$ ) was added dropwise. The reaction mixture was allowed to warm to rt, stirred for $30 \mathrm{~min}$ and quenched with $\mathrm{H}_{2} \mathrm{O}(10 \mathrm{~mL})$. The aqueous layer was diluted with sat brine $(5 \mathrm{~mL})$ and extracted with EtOAc $(3 \times 10 \mathrm{~mL})$. The combined organic layers were washed with sat brine, dried over $\mathrm{Na}_{2} \mathrm{SO}_{4}$ and concentrated in vacuo. The crude residue was purified by column chromatography (petroleum ether/EtOAc 9:1 to 7:3) to yield (+)-18a (24 mg, $31.4 \mu \mathrm{mol}, 17 \%)$ as a white foam and (-)-19a (41 mg, $53.6 \mu \mathrm{mol}, 28 \%)$ as an orange oil.

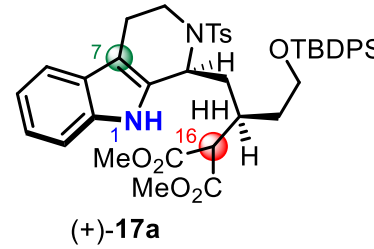

(+)-17a

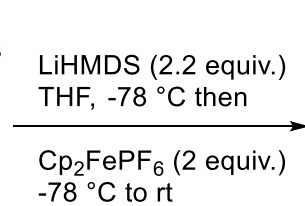

$-78^{\circ} \mathrm{C}$ to rt

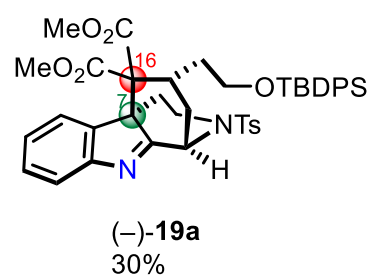

To a solution of $(+)-17 \mathrm{a}(20 \mathrm{mg}, 26.1 \mu \mathrm{mol})$ in THF $(0.5 \mathrm{~mL})$ at $-78{ }^{\circ} \mathrm{C}$ was added LiHMDS $(57 \mu \mathrm{L}, 1.0 \mathrm{M}$ in THF, $57.4 \mu \mathrm{mol}$ ) dropwise. After $30 \mathrm{~min}$ at $-78^{\circ} \mathrm{C}, \mathrm{Cp}_{2} \mathrm{FePF}_{6}$ was added solid in one portion. The reaction mixture was allowed to warm to rt, stirred for 30 min and quenched with $\mathrm{H}_{2} \mathrm{O}(2 \mathrm{~mL})$. The aqueous layer was diluted with sat brine $(1 \mathrm{~mL})$ and extracted with EtOAc $(3 \times 3 \mathrm{~mL})$. The combined organic layers were washed with sat brine, dried over $\mathrm{Na}_{2} \mathrm{SO}_{4}$ and concentrated in vacuo. The crude residue was purified by preparative TLC (petroleum ether/EtOAc 3:1) to yield (-)-19a (6 mg, $7.8 \mu \mathrm{mol}$, $30 \%)$ as an orange oil.

\section{Data for (+)-18a.}

* Rf: 0.47 (petroleum ether/EtOAc 4:1).

${ }^{*}[\alpha]_{\mathrm{D}}^{20}:+64.0\left(c=1.00, \mathrm{CH}_{2} \mathrm{Cl}_{2}\right)$.

* IR (neat): $U_{\max }\left(\mathrm{cm}^{-1}\right)=2955,2929,2857,1749,1458,1428,1264,1161,1106,911,736,702$.

* HRMS $\left(\mathrm{ESI}^{+}\right) \mathrm{m} / \mathrm{z}$ : calculated for $\mathrm{C}_{43} \mathrm{H}_{48} \mathrm{~N}_{2} \mathrm{O}_{7} \mathrm{SSiNa}^{+}[\mathrm{M}+\mathrm{Na}]^{+}$787.2844, found 787.2851.

* ${ }^{1} \mathrm{H}$ NMR (360 MHz, chloroform-d): $\delta 7.76(\mathrm{~m}, 6 \mathrm{H}), 7.45-7.39(\mathrm{~m}, 7 \mathrm{H}), 7.22(\mathrm{~d}, J=8.0 \mathrm{~Hz}, 2 \mathrm{H}), 7.13-$ $7.07(\mathrm{~m}, 3 \mathrm{H}), 4.49(\mathrm{dd}, J=12.1,3.6 \mathrm{~Hz}, 1 \mathrm{H}), 3.91-3.74(\mathrm{~m}, 3 \mathrm{H}), 3.80(\mathrm{~s}, 3 \mathrm{H}), 3.63-3.56(\mathrm{~m}, 1 \mathrm{H}), 3.52(\mathrm{~s}$, 
$3 \mathrm{H}), 3.00(\mathrm{t}, J=11.3 \mathrm{~Hz}, 1 \mathrm{H}), 2.78(\operatorname{app~d}, J=14.9 \mathrm{~Hz}, 2 \mathrm{H}), 2.45(\mathrm{dd}, J=13.5,6.7 \mathrm{~Hz}, 2 \mathrm{H}), 2.38(\mathrm{~s}, 3 \mathrm{H})$, $1.96(\operatorname{app~q}, J=12.3 \mathrm{~Hz}, 1 \mathrm{H}), 1.54-1.47(\mathrm{~m}, 1 \mathrm{H}), 1.10(\mathrm{~s}, 9 \mathrm{H})$.

${ }^{*}{ }^{13} \mathrm{C}$ NMR (91 MHz, chloroform-d): $\delta$ 168.8, 166.9, 143.7, 137.5, 136.6, 135.6 (2C), 135.6 (2C), 133.6, 133.5, 132.1, 130.1 (2C), 129.9 (2C), 128.0 (2C), 127.9 (2C), 127.7, 127.3 (2C), 122.4, 120.6, 118.4, $111.5,108.9,71.0,60.9,53.5,53.0,52.7,44.9,38.5,33.3,31.5,26.9$ (3C), 21.6, 21.3, 19.4.

\section{Data for (-)-19a.}

* Rf: 0.27 (petroleum ether/EtOAc 3:1).

$*[\alpha]_{\mathrm{D}}^{20}:-102.0\left(c=1.00, \mathrm{CH}_{2} \mathrm{Cl}_{2}\right)$.

* IR (neat): $U_{\max }\left(\mathrm{cm}^{-1}\right)=2954,2857,1738,1429,1349,1263,1227,1164,1111,1090,911,735,705$.

* HRMS (ESI $\left.{ }^{+}\right) \mathrm{m} / z$ : calculated for $\mathrm{C}_{43} \mathrm{H}_{48} \mathrm{~N}_{2} \mathrm{O}_{7} \mathrm{SSiNa}^{+}[\mathrm{M}+\mathrm{Na}]^{+}$787.2844, found 787.2840.

${ }^{*}{ }^{1}$ H NMR ( $360 \mathrm{MHz}$, chloroform- $d$ ): $\delta 7.73(\mathrm{~d}, J=8.2 \mathrm{~Hz}, 2 \mathrm{H}), 7.69-7.66(\mathrm{~m}, 4 \mathrm{H}), 7.60(\mathrm{~d}, J=7.5 \mathrm{~Hz}, 1 \mathrm{H})$, 7.40-7.38 (m, 6H), 7.32-7.27 (m, 3H), $6.86(\mathrm{t}, J=7.5 \mathrm{~Hz}, 1 \mathrm{H}), 6.33(\mathrm{~d}, J=7.5 \mathrm{~Hz}, 1 \mathrm{H}), 3.83(\mathrm{~s}, 3 \mathrm{H}), 3.74$ $(\mathrm{s}, 3 \mathrm{H}), 3.73-3.57(\mathrm{~m}, 5 \mathrm{H}), 2.47(\mathrm{~s}, 3 \mathrm{H}), 2.45-2.36(\mathrm{~m}, 2 \mathrm{H}), 2.33-2.27(\mathrm{~m}, 1 \mathrm{H}), 2.22-2.12(\mathrm{~m}, 1 \mathrm{H}), 1.91-$ $1.84(\mathrm{~m}, 1 \mathrm{H}), 1.74-1.66(\mathrm{~m}, 2 \mathrm{H}), 1.06(\mathrm{~s}, 9 \mathrm{H})$.

${ }^{*}{ }^{13}$ C NMR (91 MHz, chloroform-d): $\delta$ 177.1, 168.5, 168.1, 152.9, 143.8, 142.4, 136.7, 135.7 (4C), 133.8, 133.8, 130.0 (2C), 129.7 (2C), 128.4, 127.8 (4C), 127.3 (2C), 126.1, 121.8, 121.3, 65.5, 64.6, 62.6, 62.3, $53.3,52.7,45.4,39.8,34.4,33.9,33.2,27.0(3 C), 21.7,19.3$.

\section{Compound (-)-26a.}

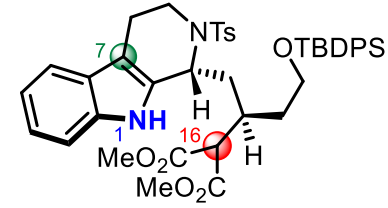

$(-)-25 a$

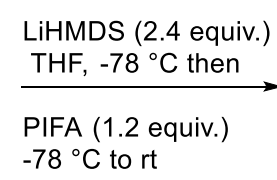

$-78^{\circ} \mathrm{C}$ to rt

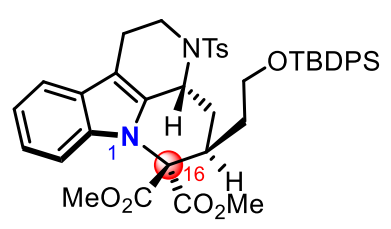

$(-)-26 \mathbf{a}$

$38 \%$

To a solution of (-)-25a (100 mg, $0.130 \mathrm{mmol})$ in THF $(2.0 \mathrm{~mL})$ at $-78{ }^{\circ} \mathrm{C}$ was added LiHMDS (339 $\mu \mathrm{L}, 1.0$ $\mathrm{M}$ in THF, $0.339 \mathrm{mmol}$ ) dropwise. After $30 \mathrm{~min}$ at $-78^{\circ} \mathrm{C}$, a solution of PIFA ( $67 \mathrm{mg}, 0.156 \mathrm{mmol}$ ) in THF $(0.5 \mathrm{~mL})$ was added dropwise. The reaction mixture was allowed to warm to rt, stirred for $15 \mathrm{~min}$ and quenched with $\mathrm{H}_{2} \mathrm{O}(5 \mathrm{~mL})$. The aqueous layer was diluted with sat brine $(3 \mathrm{~mL})$, extracted with EtOAc $(3 \times 5 \mathrm{~mL})$. The combined organic layers were washed with sat brine, dried over $\mathrm{Na}_{2} \mathrm{SO}_{4}$ and concentrated in vacuo. The crude residue was purified by column chromatography (petroleum ether $/ \mathrm{CH}_{2} \mathrm{Cl}_{2} 1: 1$ to $1: 4$ ) to yield (-)-26a (38 mg, $49.7 \mu \mathrm{mol}, 38 \%$ ) as a white foam.

* Rf: 0.31 (petroleum ether $/ \mathrm{CH}_{2} \mathrm{Cl}_{2} 2: 3$ ).

$*[\alpha]_{\mathrm{D}}^{20}:-90.8\left(c=0.87, \mathrm{CH}_{2} \mathrm{Cl}_{2}\right)$.

* IR (neat): $U_{\max }\left(\mathrm{cm}^{-1}\right)=2953,2857,1741,1455,1428,1265,1232,1162,1111,910,735,704$.

* HRMS $\left(\mathrm{ESI}^{+}\right) \mathrm{m} / \mathrm{z}$ : calculated for $\mathrm{C}_{43} \mathrm{H}_{48} \mathrm{~N}_{2} \mathrm{O}_{7} \mathrm{SSiNa}^{+}[\mathrm{M}+\mathrm{Na}]^{+}$787.2844, found 787.2826. 
* ${ }^{1} \mathrm{H}$ NMR $(360 \mathrm{MHz}$, chloroform- $d): \delta$ 7.74-7.66 (m, 6H), 7.45-7.35 (m, 7H), $7.21(\mathrm{~d}, J=8.1 \mathrm{~Hz}, 2 \mathrm{H})$, 7.14-7.06 (m, 3H), $4.55(\mathrm{dd}, J=12.4,4.5 \mathrm{~Hz}, 1 \mathrm{H}), 4.03-3.95(\mathrm{~m}, 1 \mathrm{H}), 3.90-3.85(\mathrm{~m}, 2 \mathrm{H}), 3.83(\mathrm{~s}, 3 \mathrm{H}), 3.57$ $(\mathrm{s}, 3 \mathrm{H}), 3.47$ (ddd, $J=13.7,9.1,3.6 \mathrm{~Hz}, 1 \mathrm{H}), 3.43-3.37(\mathrm{~m}, 1 \mathrm{H}), 2.74-2.65(\mathrm{~m}, 2 \mathrm{H}), 2.37(\mathrm{~s}, 3 \mathrm{H}), 2.34-2.29$ $(\mathrm{m}, 1 \mathrm{H}), 2.19(\mathrm{td}, J=12.8,3.5 \mathrm{~Hz}, 1 \mathrm{H}), 1.93-1.84(\mathrm{~m}, 1 \mathrm{H}), 1.78-1.68(\mathrm{~m}, 1 \mathrm{H}), 1.10(\mathrm{~s}, 9 \mathrm{H})$.

${ }^{*}{ }^{13}$ C NMR (91 MHz, chloroform-d): $\delta$ 168.0, 167.8, 143.7, 138.7, 137.0, 135.7 (4C), 133.8, 133.7, 131.4, 130.1 (2C), 129.9, 129.9, 128.1, 128.0 (2C), 127.9 (2C), 127.2 (2C), 122.0, 120.7, 118.2, 114.1, 110.7, $72.7,60.7,53.2,53.1,48.4,44.6,36.5,31.0,29.6,27.0$ (3C), 21.6, 21.3, 19.4 .

\section{Compound 26b.}

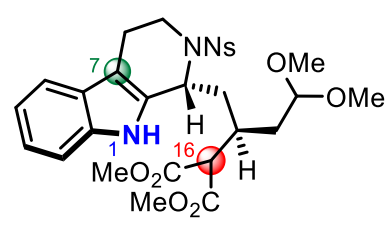

25b

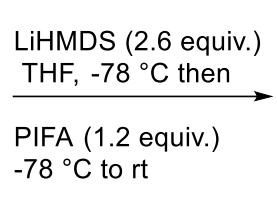

PIFA (1.2 equiv.)

$-780^{\circ}$ to

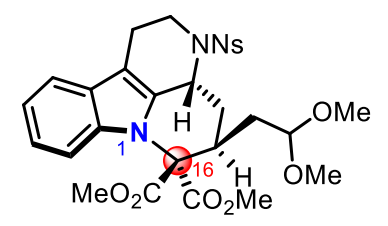

26b

To a solution of $\mathbf{2 5 b}(35 \mathrm{mg}, 0.058 \mathrm{mmol})$ in THF $(0.7 \mathrm{~mL})$ at $-78^{\circ} \mathrm{C}$ was added LiHMDS $(151 \mu \mathrm{L}, 1.0 \mathrm{M}$ in THF, $0.151 \mathrm{mmol}$ ) dropwise. After $30 \mathrm{~min}$ at $-78{ }^{\circ} \mathrm{C}$, a solution of PIFA ( $30 \mathrm{mg}, 0.069 \mathrm{mmol}$ ) in THF $(0.29 \mathrm{~mL})$ was added dropwise. The reaction mixture was allowed to warm to $\mathrm{rt}$, stirred for $15 \mathrm{~min}$ and quenched with $\mathrm{H}_{2} \mathrm{O}(5 \mathrm{~mL})$. The aqueous layer was diluted with sat brine $(3 \mathrm{~mL})$, extracted with EtOAc $\left(3 \times 5 \mathrm{~mL}\right.$ ). The combined organic layers were washed with sat brine, dried over $\mathrm{Na}_{2} \mathrm{SO}_{4}$ and concentrated in vacuo. The crude residue was purified by preparative $\mathrm{TLC}\left(\mathrm{CH}_{2} \mathrm{Cl}_{2} / \mathrm{EtOAc} 95: 5+0.1 \%\right.$ $\left.\mathrm{Et}_{3} \mathrm{~N}\right)$ to yield $\mathbf{2 6 \mathbf { b }}(13 \mathrm{mg}, 21.6 \mu \mathrm{mol}, 37 \%)$ as a yellow foam.

* Rf: $0.34 \mathrm{CH}_{2} \mathrm{Cl}_{2} / \mathrm{EtOAC}(97 / 3)$

* HRMS $\left(E S I^{+}\right) \mathrm{m} / \mathrm{z}$ : calculated for $\mathrm{C}_{28} \mathrm{H}_{32} \mathrm{~N}_{3} \mathrm{O}_{10} \mathrm{~S}^{+}[\mathrm{M}+\mathrm{H}]^{+} 602.1803$, found 602.1777

calculated for $\mathrm{C}_{28} \mathrm{H}_{31} \mathrm{~N}_{3} \mathrm{NaO}_{10} \mathrm{~S}^{+}[\mathrm{M}+\mathrm{Na}]^{+}$624.1622, found 624.1603 .

${ }^{*}{ }^{1} \mathrm{H}$ NMR (300 MHz, chloroform- $d$ ) $\delta 8.34(\mathrm{~d}, J=8.7 \mathrm{~Hz}, 2 \mathrm{H}), 8.03(\mathrm{~d}, J=8.7 \mathrm{~Hz}, 2 \mathrm{H}), 7.35$ (dd, J = 7.5, $1.5 \mathrm{~Hz}, 1 \mathrm{H}), 7.17-7.05(\mathrm{~m}, 3 \mathrm{H}), 4.84-4.74(\mathrm{~m}, 2 \mathrm{H}), 4.02(\mathrm{dt}, J=13.6,3.5 \mathrm{~Hz}, 1 \mathrm{H}), 3.94(\mathrm{~s}, 3 \mathrm{H}), 3.56(\mathrm{~s}$, $3 \mathrm{H}), 3.43(\mathrm{~s}, 3 \mathrm{H}), 3.42(\mathrm{~s}, 3 \mathrm{H}), 3.40-3.37(\mathrm{~m}, 1 \mathrm{H}), 3.16-3.24(\mathrm{~m}, 1 \mathrm{H}), 2.73(\mathrm{dt}, J=15.4,3.5 \mathrm{~Hz}, 1 \mathrm{H}), 2.60$ $(\mathrm{dt}, J=13.2,4.5 \mathrm{~Hz}, 1 \mathrm{H}), 2.38-2.16(\mathrm{~m}, 2 \mathrm{H}), 2.05-1.96(\mathrm{~m}, 1 \mathrm{H}), 1.81-1.72(\mathrm{~m}, 1 \mathrm{H})$.

${ }^{*}{ }^{13} \mathrm{C}$ NMR $(90 \mathrm{MHz}$, chloroform-d) $\delta 167.8,167.4,150.2,146.2,138.8,130.8,128.3$ (2C), 128.0, 124.8 (2C), 122.4, 121.0, 118.3, 114.1, 110.7, 102.3, 72.6, 54.1, 53.5, 53.3, 53.2, 48.5, 44.2, 36.5, 31.2, 30.1, 21.5.

\section{Compound (+)-27a.}
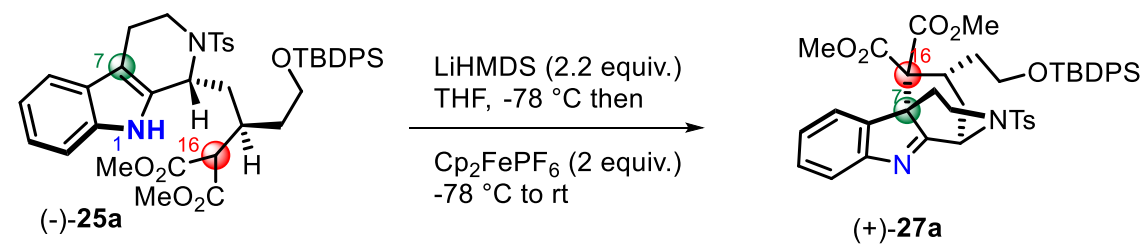

$(+)-27 a$

$45 \%$ 
To a solution of $(-)-25 \mathrm{a}(94.3 \mathrm{mg}, 0.123 \mathrm{mmol})$ in THF $(2.0 \mathrm{~mL})$ at $-78{ }^{\circ} \mathrm{C}$ was added LiHMDS $(271 \mu \mathrm{L}$, 1.0 $\mathrm{M}$ in THF, $0.2706 \mathrm{mmol}$ ) dropwise. After $30 \mathrm{~min}$ at $-78{ }^{\circ} \mathrm{C}, \mathrm{Cp}_{2} \mathrm{FePF}_{6}(81.4 \mathrm{mg}, 0.246 \mathrm{mmol}$ ) was added solid in one portion. The reaction mixture was allowed to warm to $\mathrm{rt}$, stirred for $45 \mathrm{~min}$ and quenched with sat. brine $(3 \mathrm{~mL})$ and extracted with EtOAc $(3 \times 3 \mathrm{~mL})$. The combined organic layers were dried over $\mathrm{Na}_{2} \mathrm{SO}_{4}$ and concentrated in vacuo. The crude residue was purified by preparative TLC (petroleum ether/EtOAc 7:3) to yield (+)-27a ( $45.0 \mathrm{mg}, 58.8 \mu \mathrm{mol}, 45 \%$ ) as yellow oil.

* Rf: 0.55 (petroleum ether/AcOEt 1:1).

$*[\alpha]_{\mathrm{D}}^{25}:+6.5\left(c=1.055, \mathrm{CH}_{2} \mathrm{Cl}_{2}\right)$.

* IR (neat): $U_{\max }\left(\mathrm{cm}^{-1}\right)=2928,2856,1736,1574,1429,1351,1247,1209,1166,1110,1044,821,704$, 629.

* HRMS $\left(\mathrm{ESI}^{+}\right) \mathrm{m} / \mathrm{z}$ : calculated for $\mathrm{C}_{43} \mathrm{H}_{49} \mathrm{~N}_{2} \mathrm{O}_{7} \mathrm{SSi}^{+}[\mathrm{M}+\mathrm{H}]^{+}$765.3024, found 765.2985.

${ }^{*}{ }^{1} \mathrm{H}$ NMR $(360 \mathrm{MHz}$, chloroform- $d$ ): $87.80-7.75(\mathrm{~m}, 6 \mathrm{H}), 7.58(\mathrm{~d}, J=7.5 \mathrm{~Hz}, 1 \mathrm{H}), 7.46-7.40(\mathrm{~m}, 6 \mathrm{H}), 7.37$ $(\mathrm{d}, J=8.1 \mathrm{~Hz}, 2 \mathrm{H}), 7.22(\mathrm{t}, J=7.5 \mathrm{~Hz}, 1 \mathrm{H}), 6.76(\mathrm{t}, J=7.5 \mathrm{~Hz}, 1 \mathrm{H}), 5.62(\mathrm{~d}, J=7.5 \mathrm{~Hz}, 1 \mathrm{H}), 3.90-3.80(\mathrm{~m}$, $3 \mathrm{H}), 3.80(\mathrm{~s}, 3 \mathrm{H}), 3.76(\mathrm{~s}, 3 \mathrm{H}), 3.73(\mathrm{~d}, J=4.8 \mathrm{~Hz}, 1 \mathrm{H}), 3.36-3.26(\mathrm{~m}, 2 \mathrm{H}), 2.51(\mathrm{~s}, 3 \mathrm{H}), 2.42-2.34(\mathrm{~m}, 3 \mathrm{H})$, 1.92-1.83 (m, $1 \mathrm{H}), 1.64(\mathrm{dd}, J=13.3,5.5 \mathrm{~Hz}, 1 \mathrm{H}), 1.44-1.32(\mathrm{~m}, 1 \mathrm{H}), 1.11(\mathrm{~s}, 9 \mathrm{H})$.

${ }^{*}{ }^{13} \mathrm{C}$ NMR $(100 \mathrm{MHz}$, chloroform-d): $\delta$ 179.0, 168.8, 168.5, 153.5, 144.3, 144.1, 135.8 (2C), 135.7 (2C), 134.0, 133.7, 132.1, 130.0 (2C), 129.7, 129.6, 128.5 (2C), 128.3, 127.9 (2C), 127.8 (2C), 126.2, 121.8, $120.4,63.5,63.2,61.7,61.3,53.5,52.9,49.1,34.0,33.1,32.8,32.6,26.9$ (3C), 21.7, 19.4.

\section{Compounds (+)-28a and (+)-28b.}

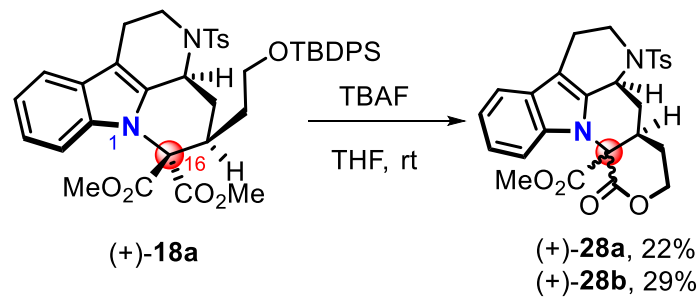

To a solution of (+)-18a (31.8 mg, $41.5 \mu \mathrm{mol})$ in THF $(1.0 \mathrm{~mL})$ at $0{ }^{\circ} \mathrm{C}$ was added TBAF. $3 \mathrm{H}_{2} \mathrm{O}(19.6 \mathrm{mg}$, $62.2 \mu \mathrm{mol})$. The reaction mixture was stirred for $2 \mathrm{~h}$ and quenched with a saturated aqu. solution of $\mathrm{NaHCO}_{3}(3 \mathrm{~mL})$ and extracted with EtOAc $(3 \times 3 \mathrm{~mL})$. The combined organic layers were dried over $\mathrm{Na}_{2} \mathrm{SO}_{4}$ and concentrated in vacuo. The crude residue was purified by preparative TLC (petroleum ether/EtOAc 2:3) to yield (+)-28a (4.5 mg, $9.11 \mu \mathrm{mol}, 22 \%$ ) as yellow oil and (+)-28b (6.0 mg, $12.1 \mu \mathrm{mol}$, $29 \%)$ as yellow oil.

\section{Data for (+)-28a.}

* Rf: 0.30 (petroleum ether/EtOAc 1:1).

$*[\alpha]_{\mathbf{D}}^{25}:+14.3\left(c=0.033, \mathrm{CH}_{2} \mathrm{Cl}_{2}\right)$.

* IR (neat): $U_{\max }\left(\mathrm{cm}^{-1}\right)=2924,2852,1738,1456,1309,1262,1216,1163,1096,801,747,665$.

* HRMS $\left(\mathrm{ESI}^{+}\right) \mathrm{m} / \mathrm{z}$ : calculated for $\mathrm{C}_{26} \mathrm{H}_{27} \mathrm{~N}_{2} \mathrm{O}_{6} \mathrm{~S}[\mathrm{M}+\mathrm{H}]^{+} 495.1584$, found 495.1564 .

* ${ }^{1} \mathrm{H}$ NMR (300 MHz, chloroform-d): $\delta 7.74$ (d, $J=8.3 \mathrm{~Hz}, 2 \mathrm{H}$ ), 7.47 (dd, $\left.J=7.2,1.5 \mathrm{~Hz}, 1 \mathrm{H}\right), 7.34-7.28$ $(\mathrm{m}, 3 \mathrm{H}), 7.17-7.08(\mathrm{~m}, 2 \mathrm{H}), 4.68(\mathrm{dd}, J=10.8,4.3 \mathrm{~Hz}, 1 \mathrm{H}), 4.61-4.52(\mathrm{~m}, 2 \mathrm{H}), 3.96(\mathrm{dt}, J=13.5,3.7 \mathrm{~Hz}$, 
$1 \mathrm{H}), 3.50(\mathrm{~s}, 3 \mathrm{H}), 3.39$ (ddd, $J=13.5,9.8,3.4 \mathrm{~Hz}, 1 \mathrm{H}), 2.87-2.77(\mathrm{~m}, 1 \mathrm{H}), 2.63(\mathrm{dt}, J=15.4,3.4 \mathrm{~Hz}, 1 \mathrm{H})$, 2.53-2.43 (m, 3H), $2.41(\mathrm{~s}, 3 \mathrm{H}), 2.29-2.19(\mathrm{~m}, 1 \mathrm{H}), 1.94-1.80(\mathrm{~m}, 1 \mathrm{H})$.

${ }^{*}{ }^{13}$ C NMR (100 MHz, chloroform-d): $\delta$ 167.5, 166.1, 144.0, 139.3, 137.1, 133.0, 130.2 (2C), 129.2, 127.1 (2C), 123.1, 121.6, 118.2, 114.3, 112.1, 70.2, 65.6, 53.0, 52.1, 43.5, 37.4, 32.0, 25.4, 21.7, 21.0.

\section{Data for $(+)-28 b$.}

* Rf: 0.25 (petroleum ether/EtOAc 1:1).

$*[\alpha]_{\mathrm{D}}^{24}:+1.0\left(c=0.27, \mathrm{CH}_{2} \mathrm{Cl}_{2}\right)$.

* IR (neat): $U_{\max }\left(\mathrm{cm}^{-1}\right)=2923,2851,1742,1455,1310,1268,1090,903,813,740,664$.

* HRMS (ESI $\left.{ }^{+}\right) \mathrm{m} / \mathrm{z}$ : calculated for $\mathrm{C}_{26} \mathrm{H}_{27} \mathrm{~N}_{2} \mathrm{O}_{6} \mathrm{~S}^{+}[\mathrm{M}+\mathrm{H}]^{+}$495.1584, found 495.1562.

$*{ }^{1}$ H NMR $(360 \mathrm{MHz}$, chloroform- $d$ ): $\delta 7.75(\mathrm{~d}, J=8.2 \mathrm{~Hz}, 2 \mathrm{H}), 7.35$ (d, $J=7.8 \mathrm{~Hz}, 1 \mathrm{H}), 7.31(\mathrm{~d}, J=8.2$ $\mathrm{Hz}, 2 \mathrm{H}), 7.18-7.06(\mathrm{~m}, 3 \mathrm{H}), 4.66(\mathrm{dd}, J=11.5,4.0 \mathrm{~Hz}, 1 \mathrm{H}), 4.56-4.31(\mathrm{~m}, 2 \mathrm{H}), 3.97(\mathrm{~s}, 3 \mathrm{H}), 3.91-3.82(\mathrm{~m}$, $1 \mathrm{H}), 3.43$ (ddd, $J=13.0,9.1,3.5 \mathrm{~Hz}, 1 \mathrm{H}), 3.19-3.12(\mathrm{~m}, 1 \mathrm{H}), 2.77-2.72(\mathrm{~m}, 1 \mathrm{H}), 2.68(\mathrm{dt}, J=15.5,3.5 \mathrm{~Hz}$, $1 \mathrm{H}), 2.42(\mathrm{~s}, 3 \mathrm{H}), 2.41-2.35(\mathrm{~m}, 1 \mathrm{H}), 2.31-2.21(\mathrm{~m}, 1 \mathrm{H}), 2.06-1.92(\mathrm{~m}, 2 \mathrm{H})$.

${ }^{*}{ }^{13}$ C NMR (100 MHz, chloroform-d): $\delta$ 169.4, 165.3, 144.1, 138.1, 136.6, 130.2 (2C), 129.9, 127.3 (3C), $122.7,120.7,118.5,112.6,110.4,69.3,66.2,54.2,52.1,44.8,37.3,33.8,26.5,21.7,21.1$.

\section{Compound (-)-29.}

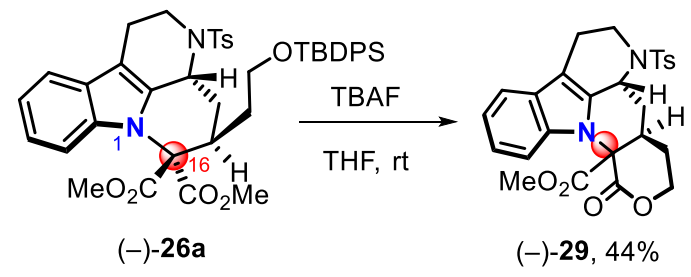

To a solution of (-)-26a (103.0 mg, $0.135 \mathrm{mmol})$ in THF $(2.7 \mathrm{~mL})$ at rt was added TBAF (175 $\mu \mathrm{L}, 1.0 \mathrm{M}$ in THF, $0.175 \mathrm{mmol})$. The reaction mixture was stirred for $2 \mathrm{~h}$ and quenched with $\mathrm{H}_{2} \mathrm{O}(2 \mathrm{~mL})$. The aqueous layer was diluted with sat brine $(3 \mathrm{~mL})$, extracted with EtOAc $(3 \times 5 \mathrm{~mL})$. The combined organic layers were washed with sat brine, dried over $\mathrm{Na}_{2} \mathrm{SO}_{4}$ and concentrated in vacuo. The combined organic layers were dried over $\mathrm{Na}_{2} \mathrm{SO}_{4}$ and concentrated in vacuo. The crude residue was purified by preparative TLC (petroleum ether/EtOAc 3:2) to yield (-)-29 (29.0 mg, $58.7 \mu \mathrm{mol}, 43 \%)$ as a white solid.

* Rf: 0.41 (petroleum ether/EtOAc 3:2).

$*[\boldsymbol{\alpha}]_{\mathbf{D}}^{24}:-51.3\left(c=0.37, \mathrm{CH}_{2} \mathrm{Cl}_{2}\right)$.

* IR (neat): $U_{\max }\left(\mathrm{cm}^{-1}\right)=2954,2923,2852,1747,1456,1428,1337,1266,1162,1094,912,730$.

* HRMS $\left(\mathrm{ESI}^{+}\right) \mathrm{m} / \mathrm{z}$ : calculated for $\mathrm{C}_{26} \mathrm{H}_{26} \mathrm{~N}_{2} \mathrm{O}_{6} \mathrm{SNa}^{+}[\mathrm{M}+\mathrm{Na}]^{+}$517.1404, found 517.1398.

* ${ }^{1} \mathrm{H}$ NMR (300 MHz, chloroform-d): $7.73(\mathrm{~d}, J=8.5 \mathrm{~Hz}, 2 \mathrm{H}), 7.39$ (dd, $\left.J=6.9,1.5 \mathrm{~Hz}, 1 \mathrm{H}\right), 7.35-7.30(\mathrm{~m}$, $3 \mathrm{H}), 7.18-7.11(\mathrm{~m}, 2 \mathrm{H}), 4.67(\mathrm{dd}, J=12.1,4.0 \mathrm{~Hz}, 1 \mathrm{H}), 4.51-4.40(\mathrm{~m}, 2 \mathrm{H}), 3.83(\mathrm{dt}, J=13.4,4.0 \mathrm{~Hz}, 1 \mathrm{H})$, $3.73(\mathrm{~s}, 3 \mathrm{H}), 3.49-3.42(\mathrm{~m}, 1 \mathrm{H}), 3.37-3.30(\mathrm{~m}, 1 \mathrm{H}), 2.74(\mathrm{dt}, J=15.2,4.0 \mathrm{~Hz}, 1 \mathrm{H}), 2.63(\mathrm{dd}, J=13.0,4.0$ $\mathrm{Hz}, 1 \mathrm{H}), 2.46-2.34(\mathrm{~m}, 2 \mathrm{H}), 2.41(\mathrm{~s}, 3 \mathrm{H}), 2.22-2.13(\mathrm{~s}, 1 \mathrm{H}), 1.97(\mathrm{td}, J=13.0,4.0 \mathrm{~Hz}, 1 \mathrm{H})$.

${ }^{*}{ }^{13}$ C NMR (91 MHz, chloroform-d): $\delta$ 168.5, 166.9, 144.1, 138.9, 136.6, 130.6, 130.2 (2C), 127.5, 127.1 (2C), 122.9, 120.9, 118.3, 112.5, 110.0, 67.9, 67.6, 53.8, 48.1, 44.1, 36.6, 33.0, 27.4, 21.6, 21.2. 
IV. NMR spectra of compounds

${ }^{1} \mathrm{H} \mathrm{NMR}\left(360 \mathrm{MHz}, \mathrm{CDCl}_{3}\right)$

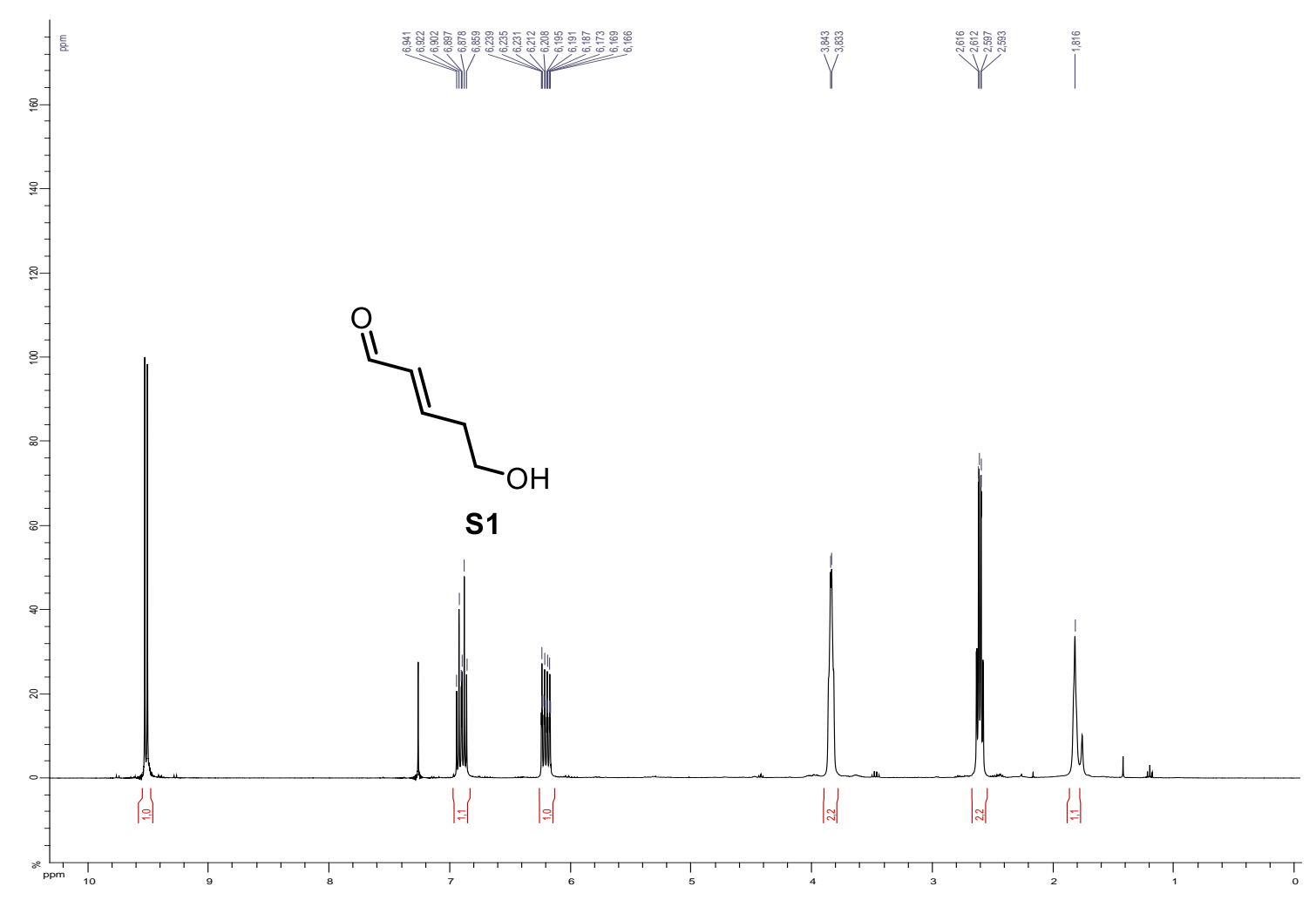

${ }^{1} \mathrm{H}$ NMR $\left(360 \mathrm{MHz}, \mathrm{CDCl}_{3}\right.$ )

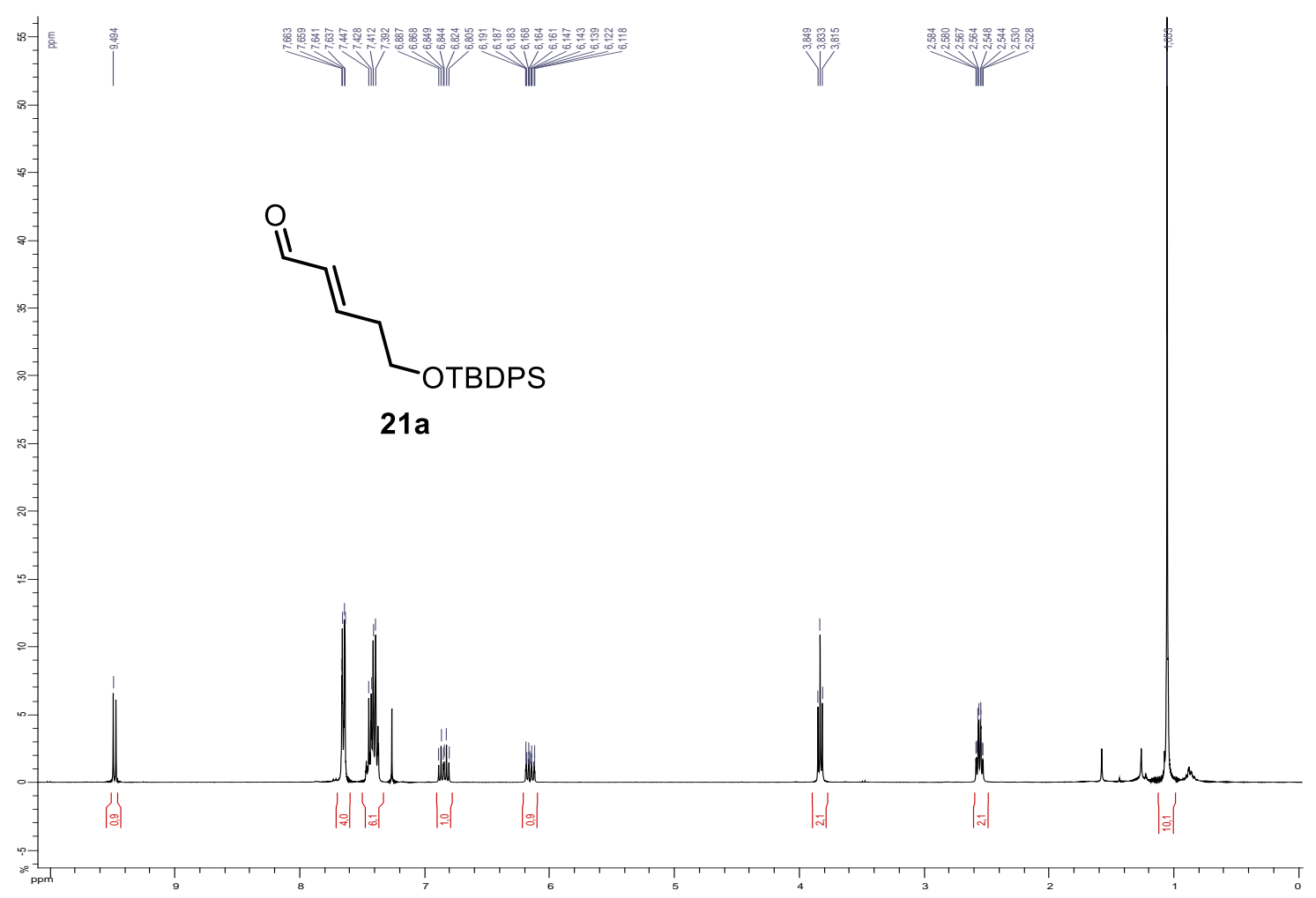


${ }^{1} \mathrm{H}$ NMR $\left(300 \mathrm{MHz}, \mathrm{CDCl}_{3}\right.$ )

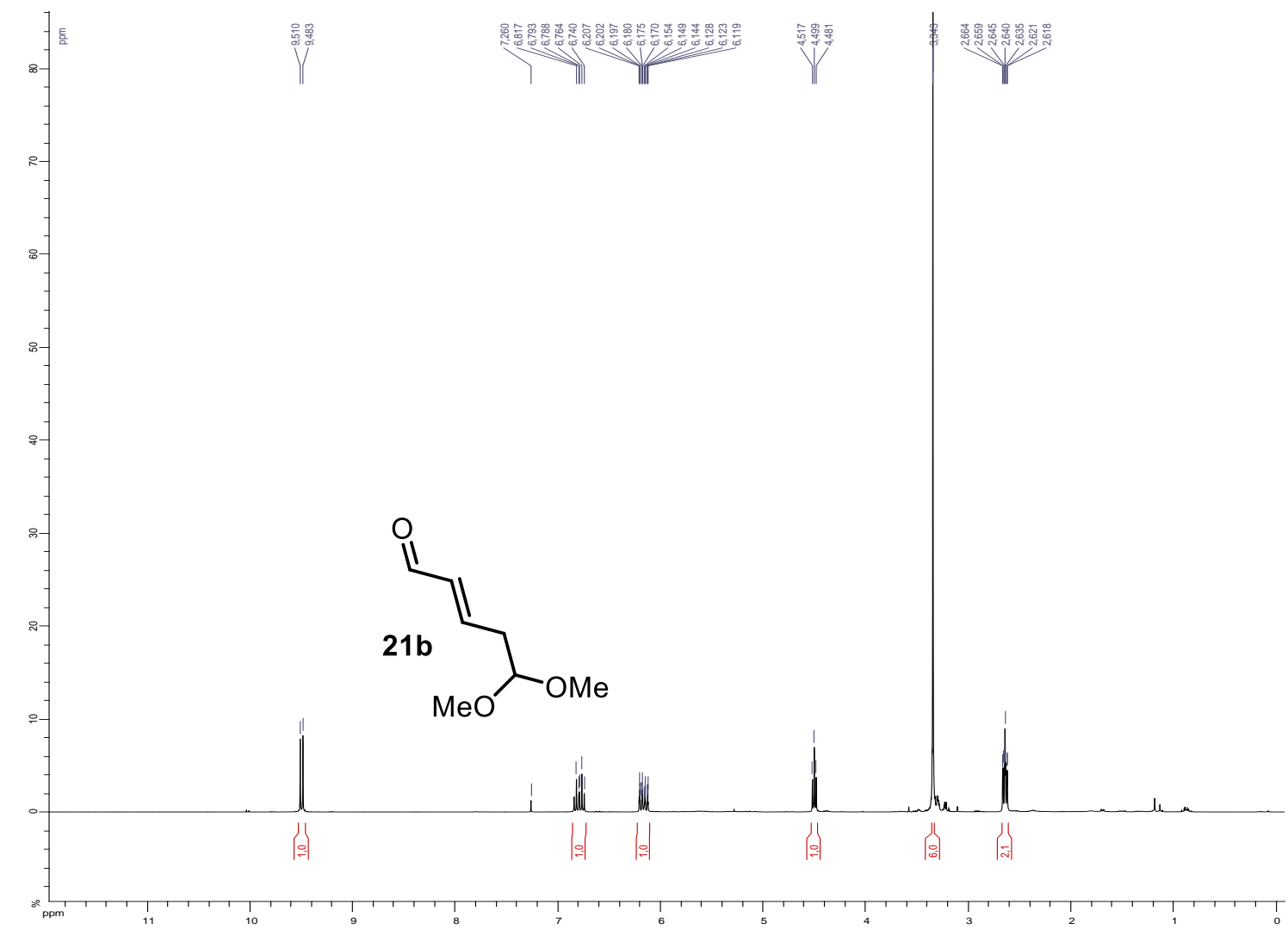

${ }^{13} \mathrm{C} \mathrm{NMR}\left(75 \mathrm{MHz}, \mathrm{CDCl}_{3}\right)$

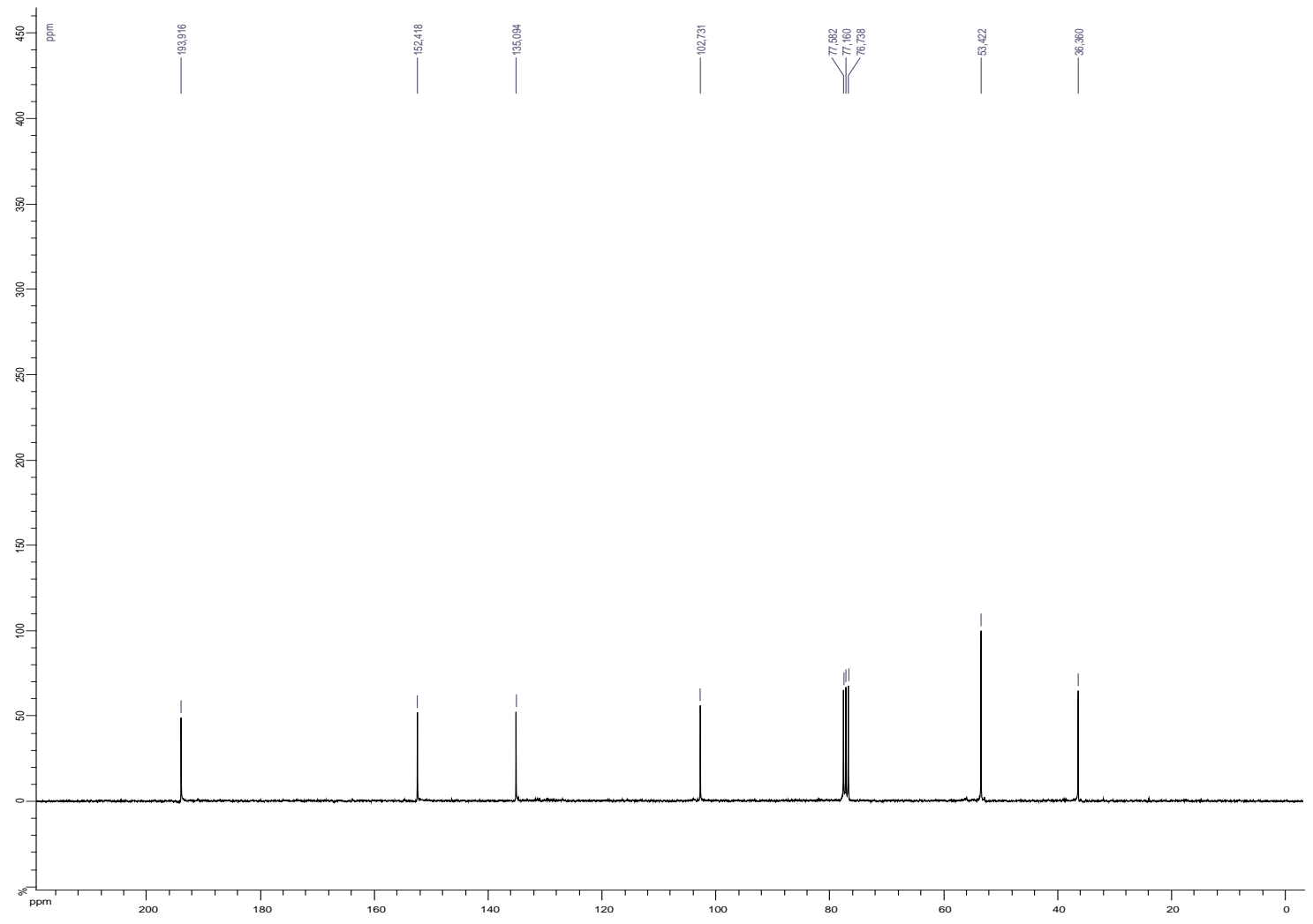

S15 
${ }^{1} \mathrm{H}$ NMR $\left(360 \mathrm{MHz}, \mathrm{CDCl}_{3}\right)$

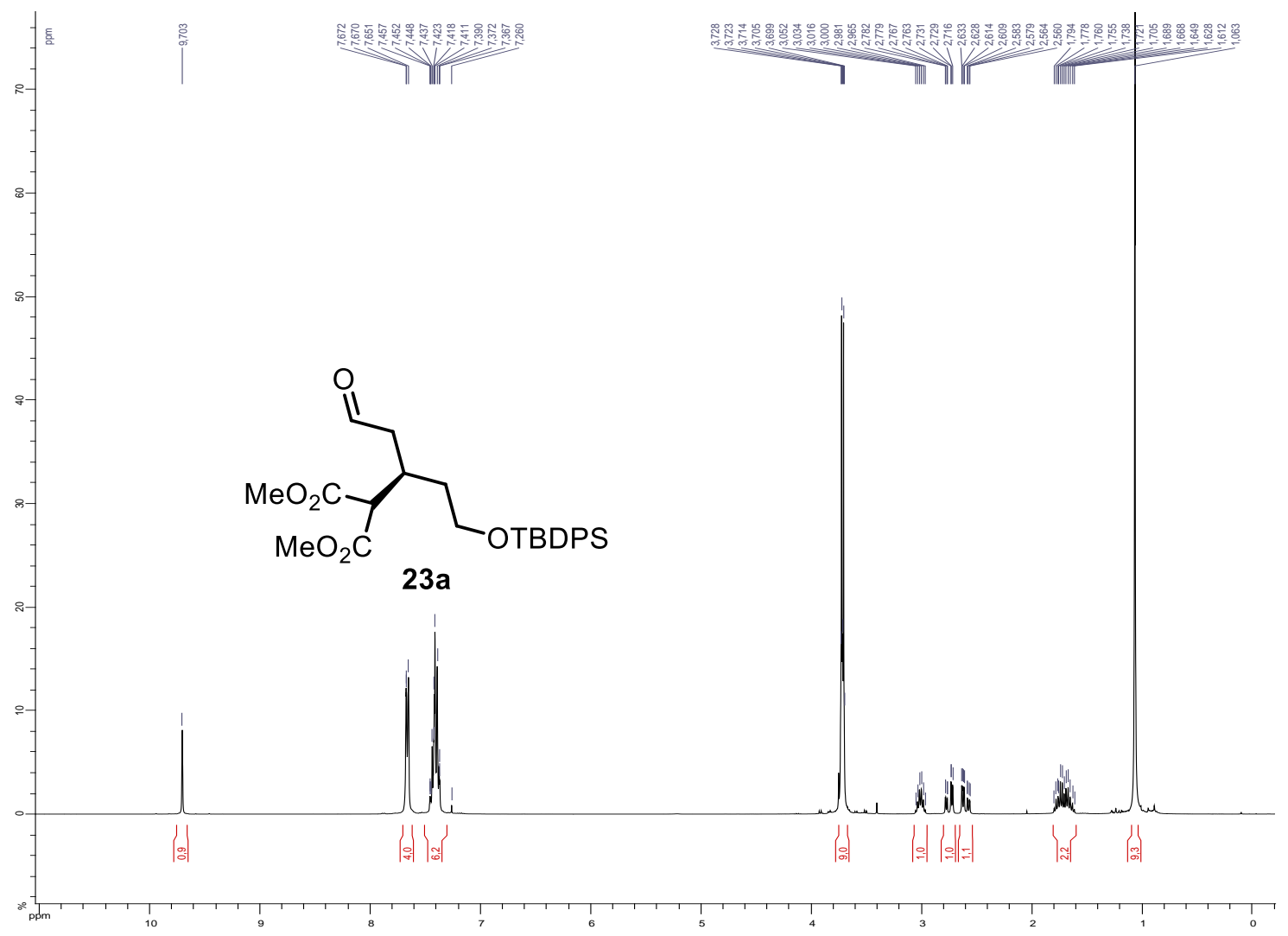

${ }^{13} \mathrm{C}$ NMR (91 MHz, $\mathrm{CDCl}_{3}$ )

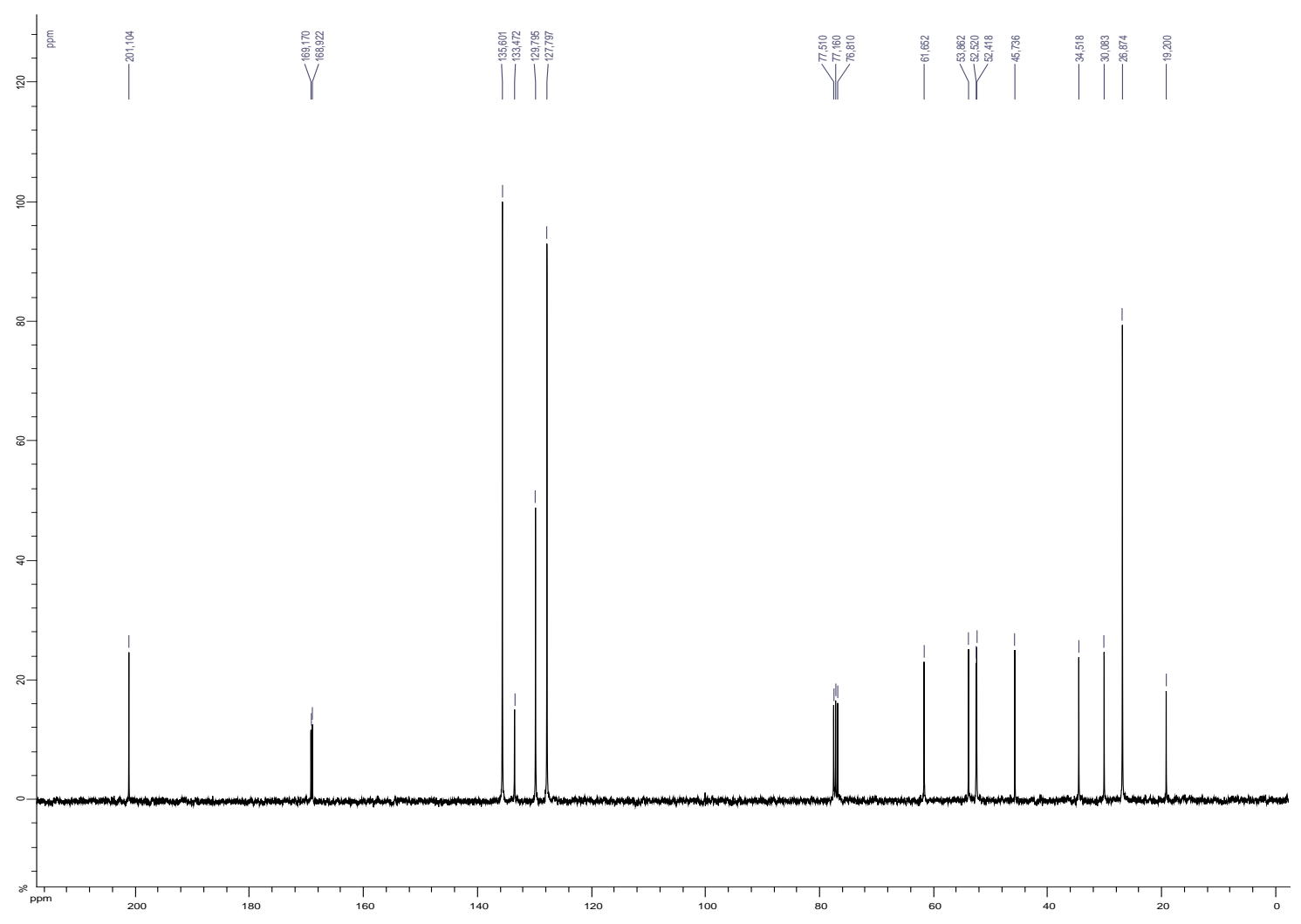


${ }^{1} \mathrm{H}$ NMR $\left(360 \mathrm{MHz}, \mathrm{CDCl}_{3}\right)$

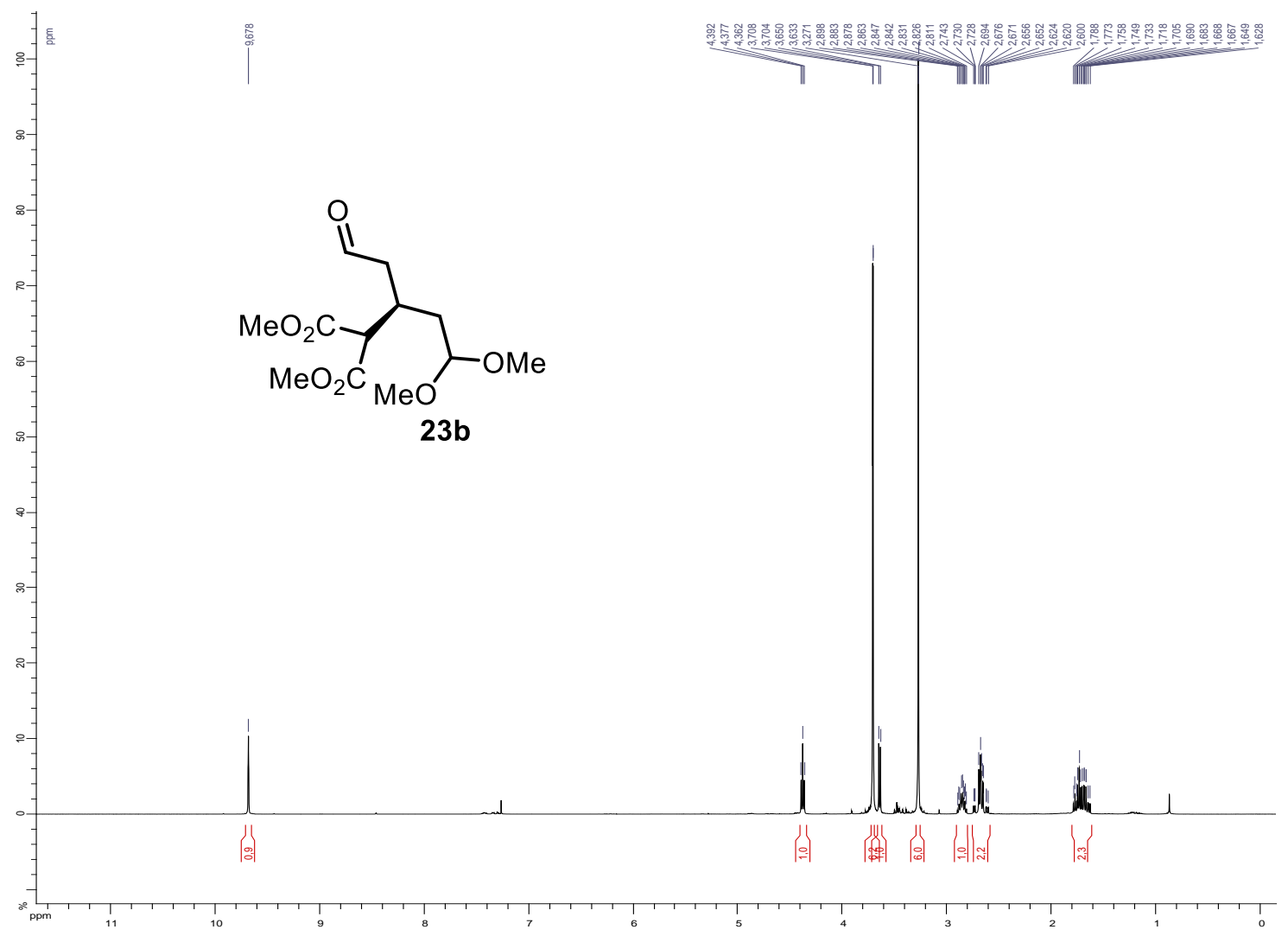

${ }^{13} \mathrm{C}$ NMR (91 MHz, $\mathrm{CDCl}_{3}$ )

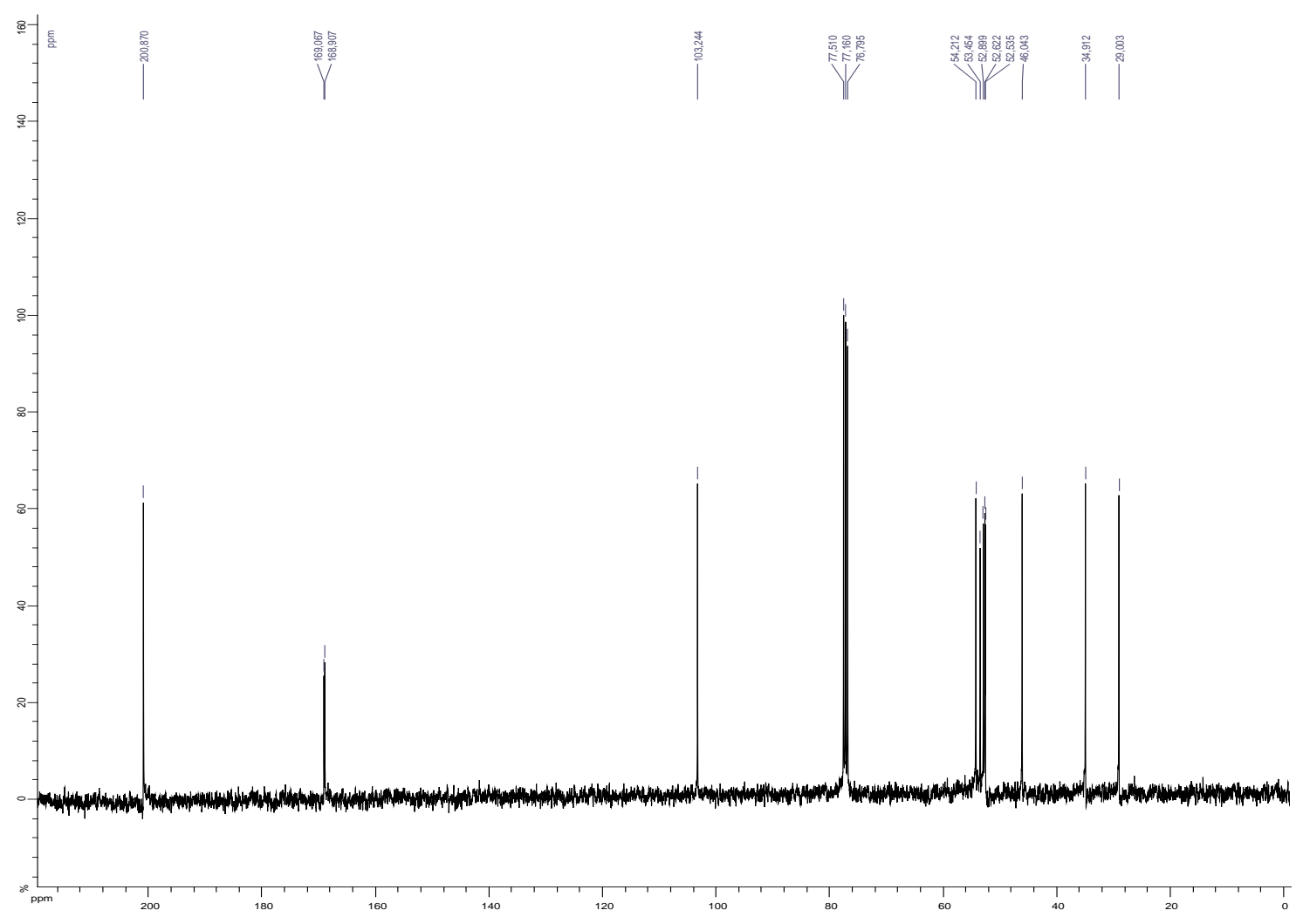


${ }^{1} \mathrm{H}$ NMR $\left(360 \mathrm{MHz}, \mathrm{CDCl}_{3}\right)$

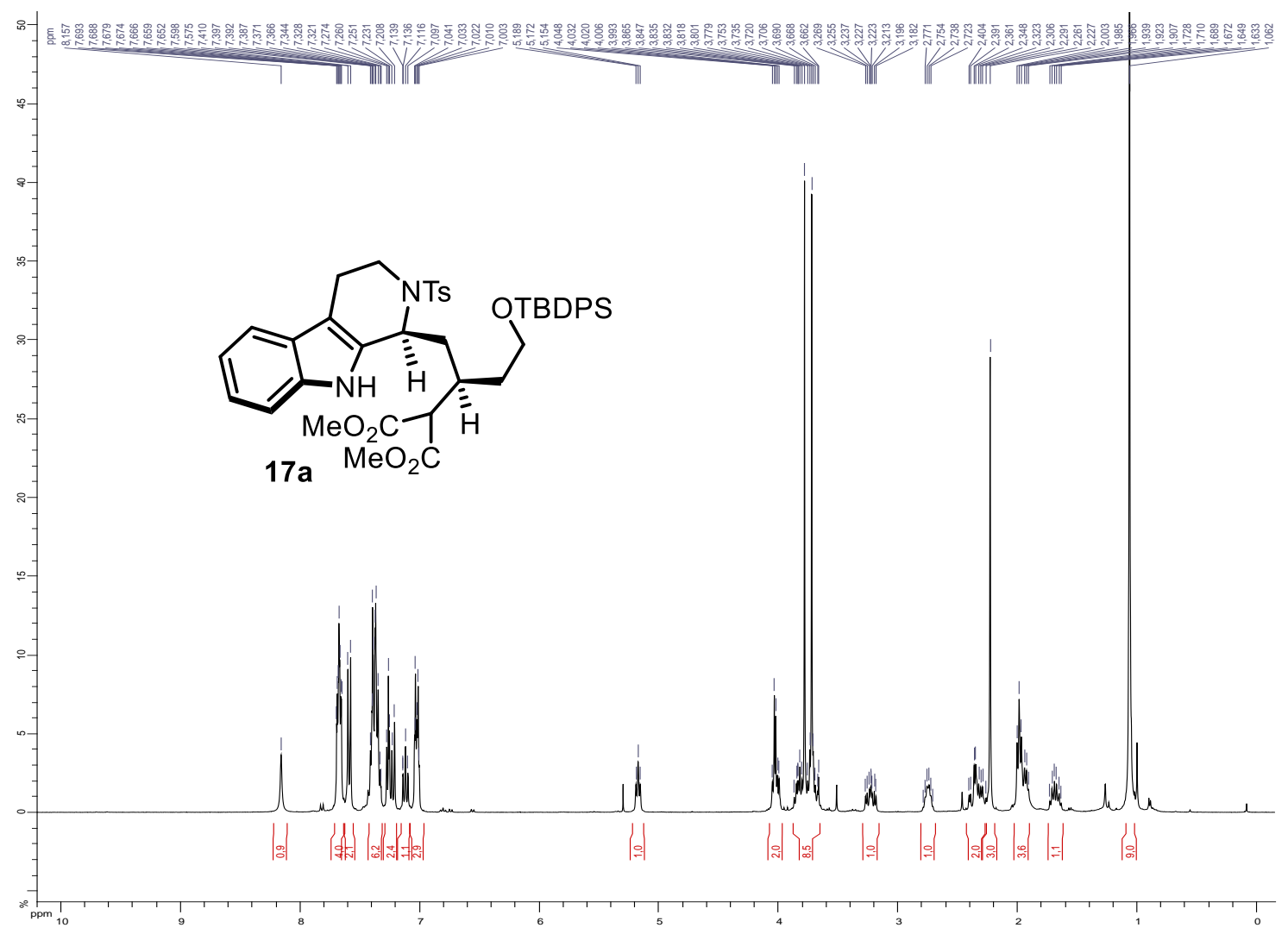

${ }^{13} \mathrm{C} \mathrm{NMR}\left(63 \mathrm{MHz}, \mathrm{CDCl}_{3}\right)$

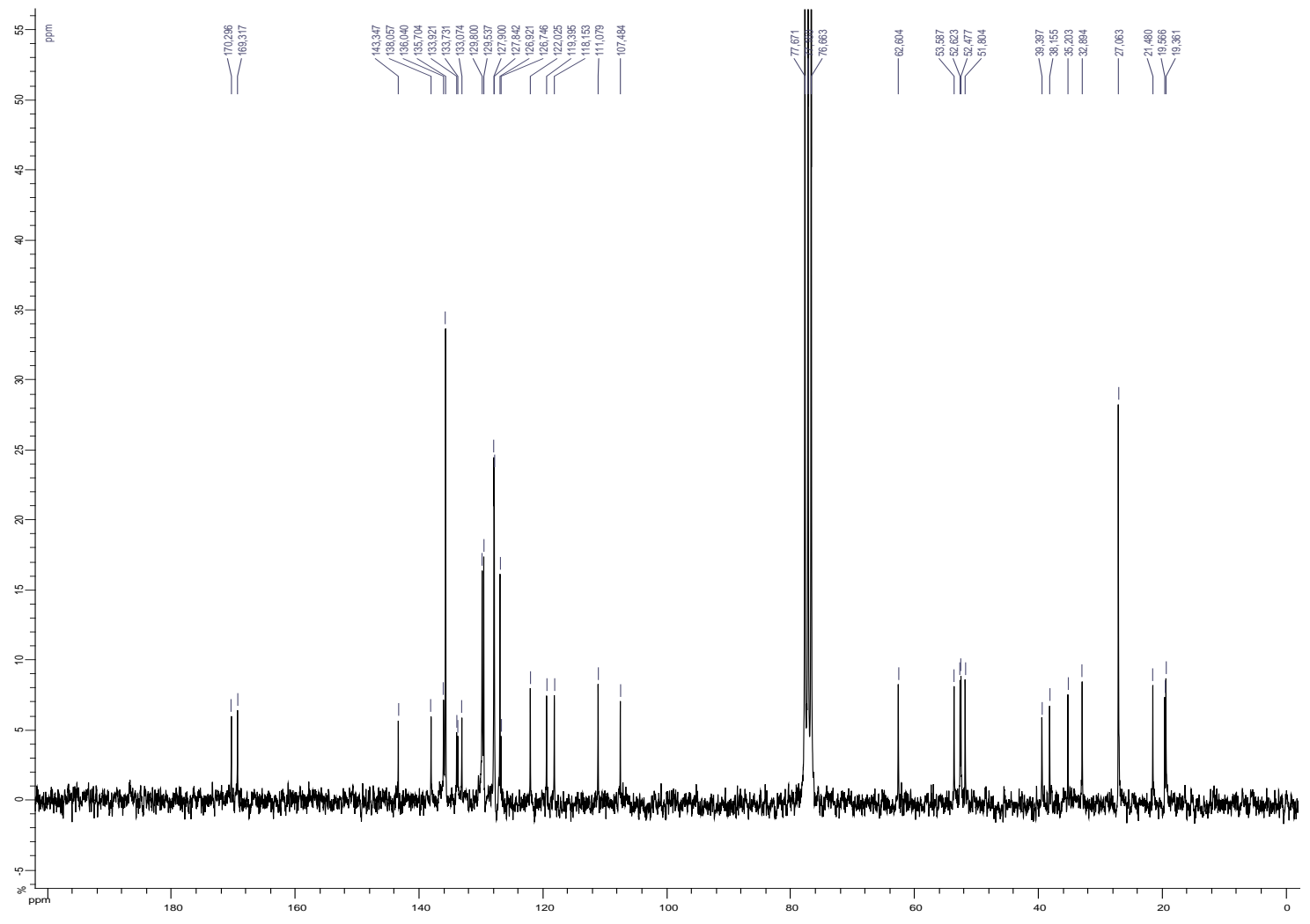


${ }^{1} \mathrm{H}$ NMR $\left(300 \mathrm{MHz}, \mathrm{CDCl}_{3}\right)$

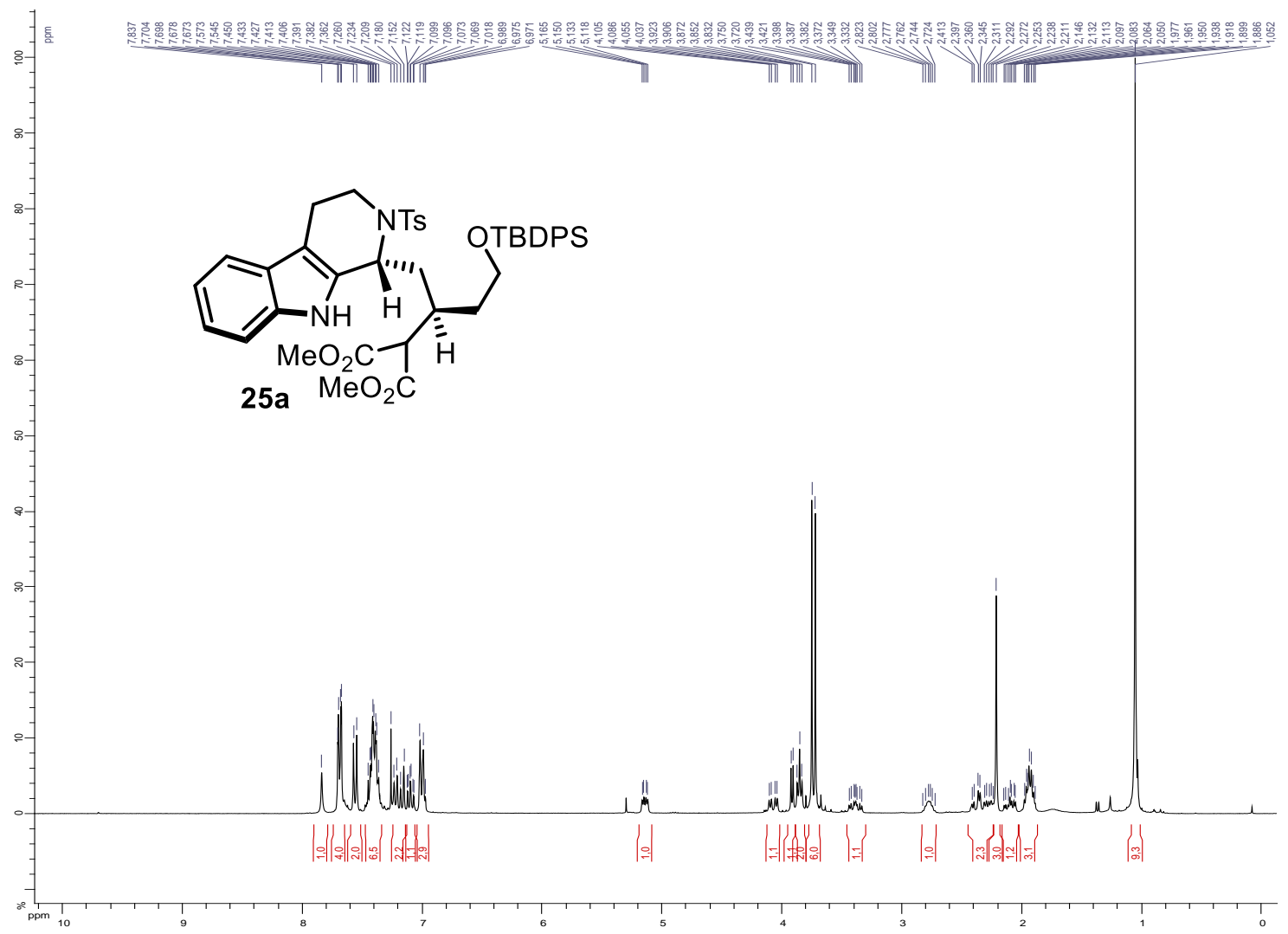

${ }^{13} \mathrm{C}$ NMR (63 MHz, $\mathrm{CDCl}_{3}$ )

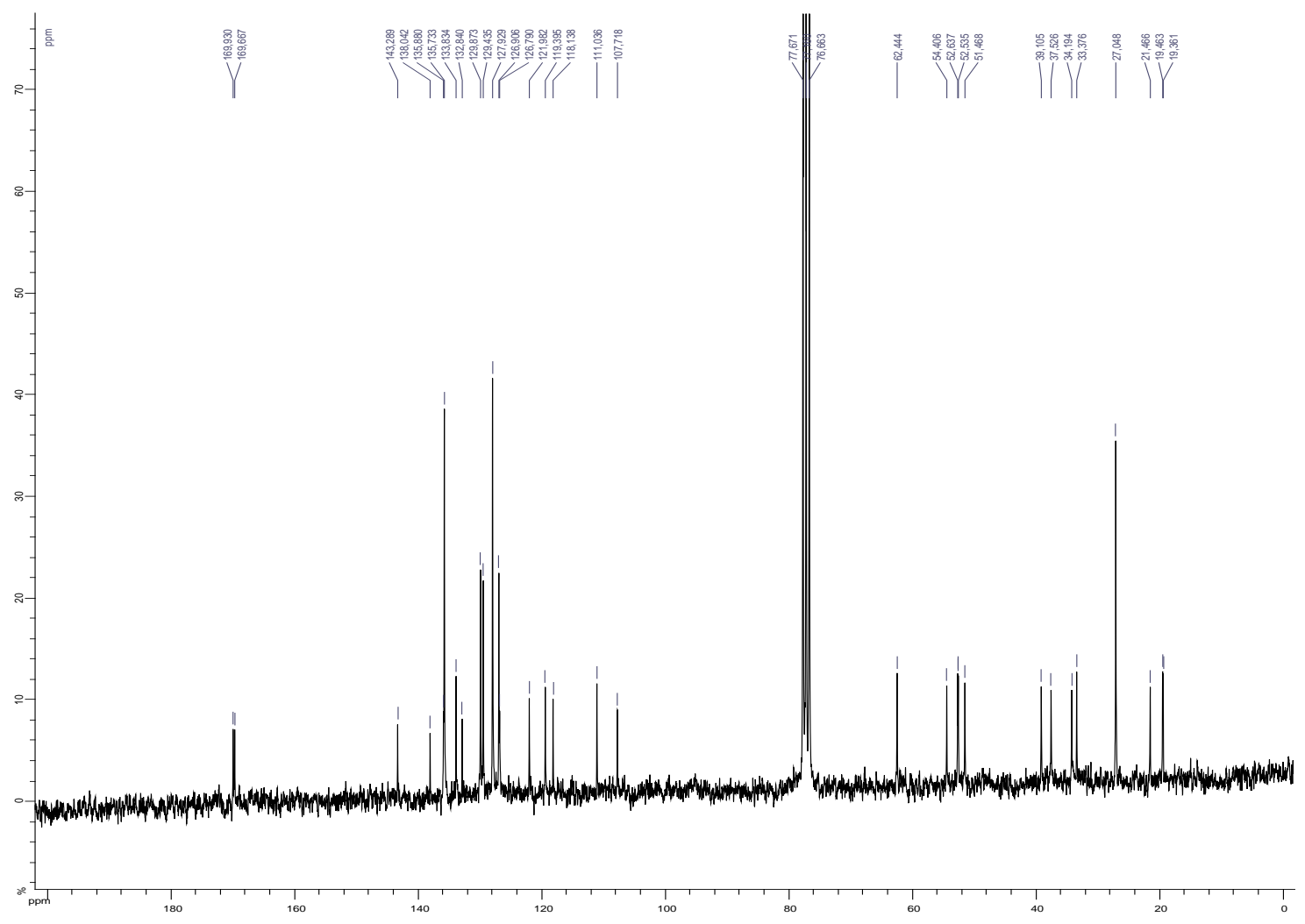


${ }^{1} \mathrm{H}$ NMR $\left(300 \mathrm{MHz}, \mathrm{CDCl}_{3}\right)$

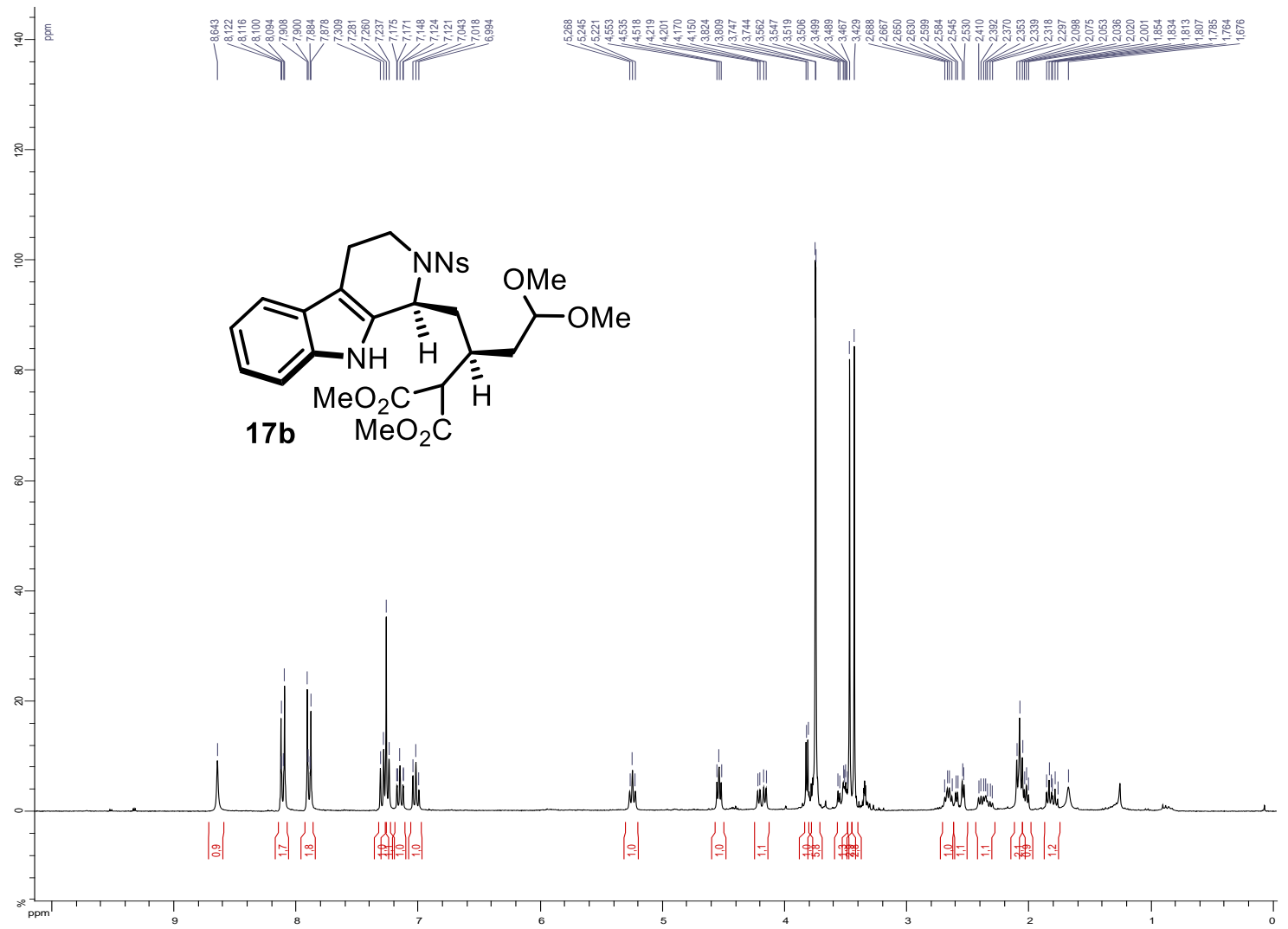

${ }^{13} \mathrm{C}$ NMR $\left(63 \mathrm{MHz}, \mathrm{CDCl}_{3}\right)$

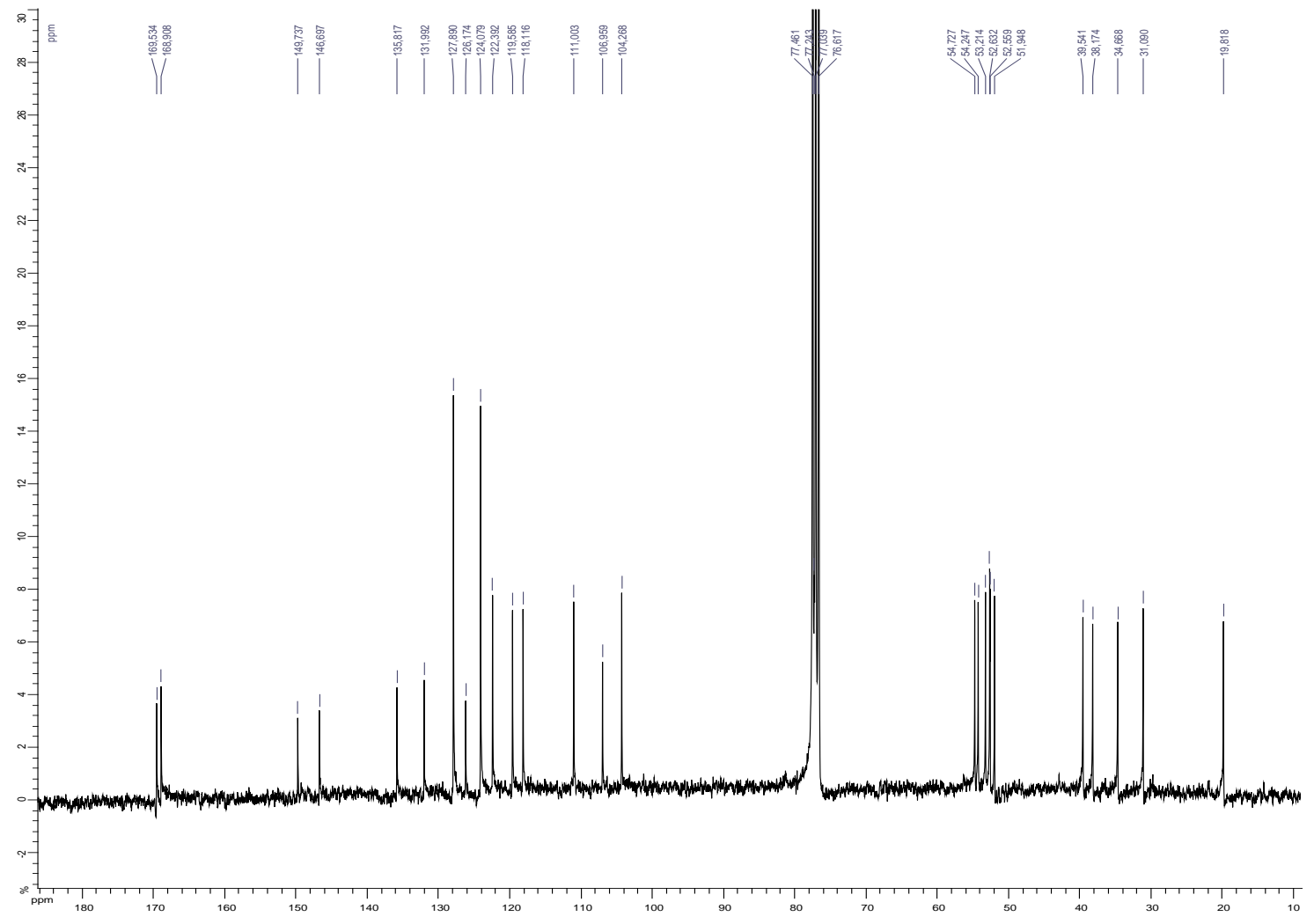


${ }^{1} \mathrm{H}$ NMR $\left(360 \mathrm{MHz}, \mathrm{CDCl}_{3}\right)$

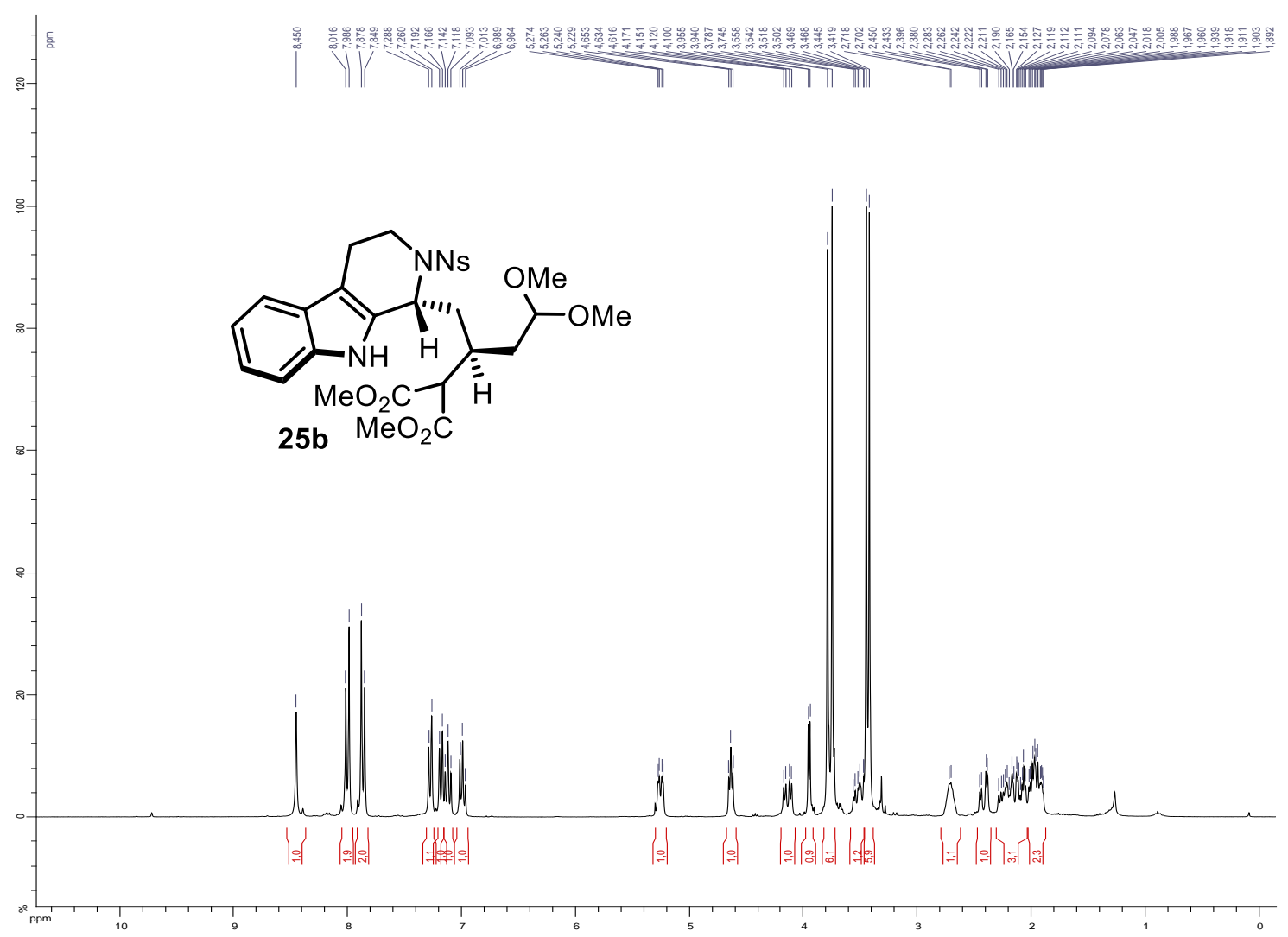

${ }^{13} \mathrm{C}$ NMR (91 MHz, $\mathrm{CDCl}_{3}$ )

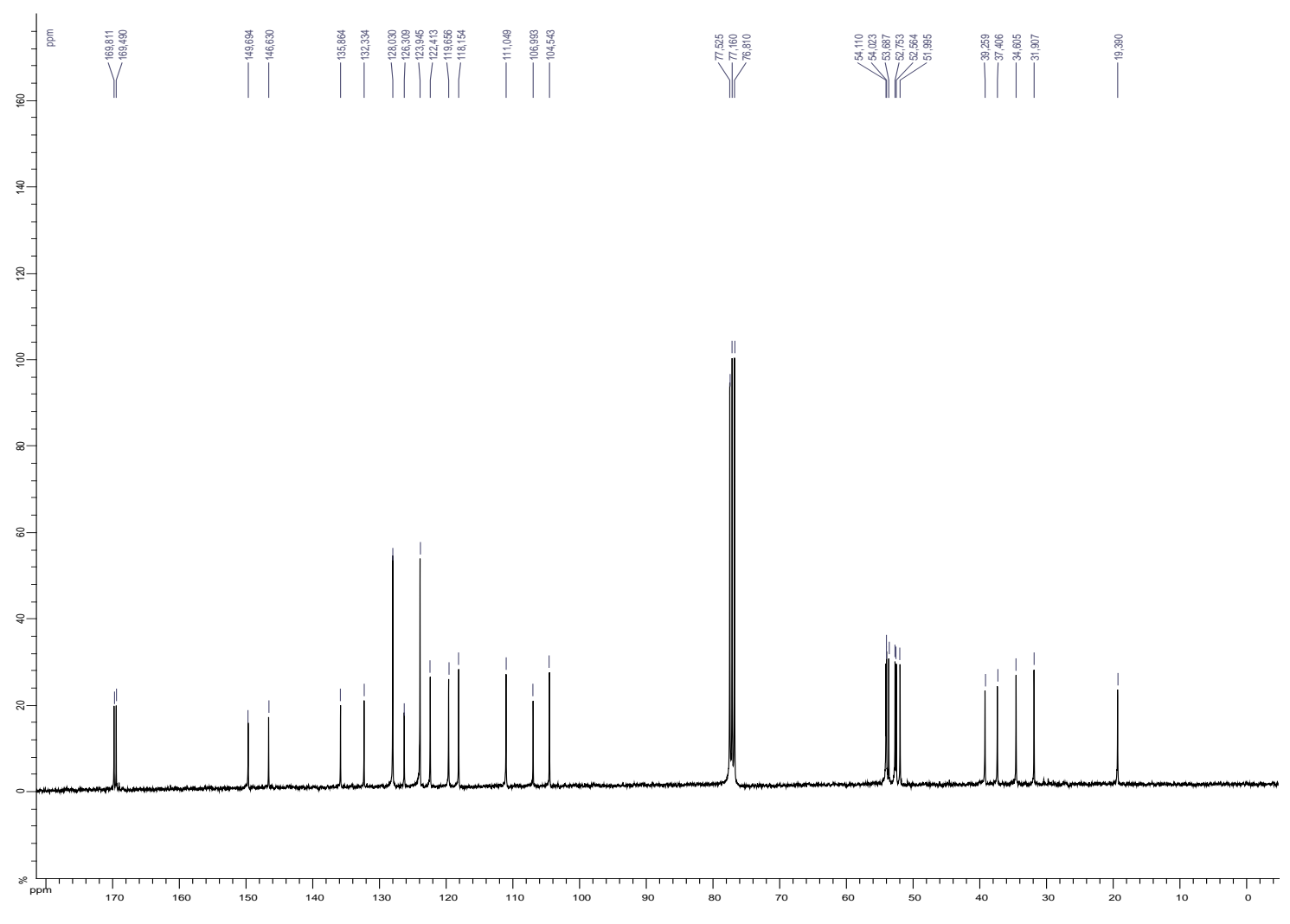


${ }^{1} \mathrm{H}$ NMR $\left(360 \mathrm{MHz}, \mathrm{CDCl}_{3}\right)$

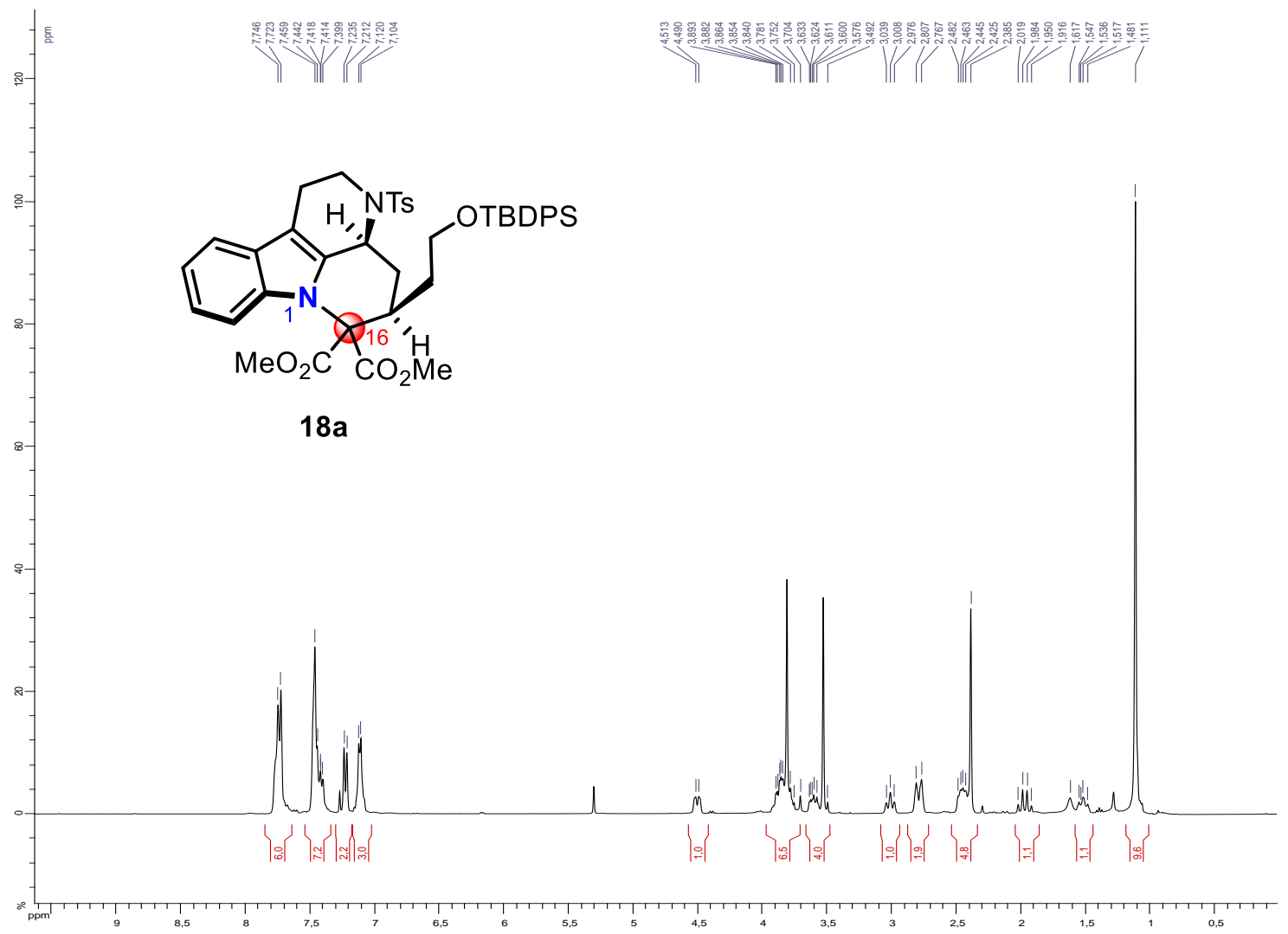

${ }^{13} \mathrm{C}$ NMR (91 MHz, $\left.\mathrm{CDCl}_{3}\right)$

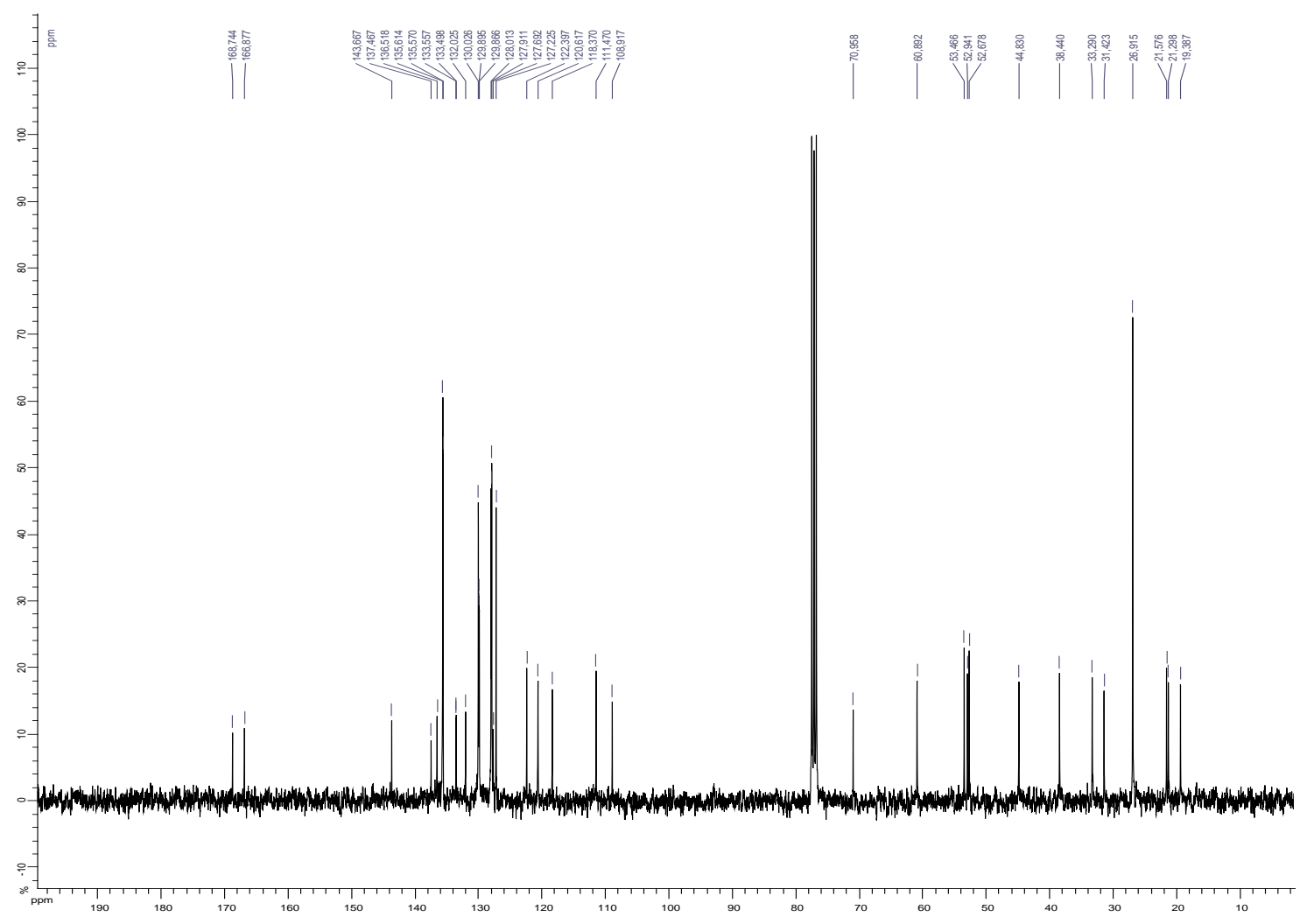




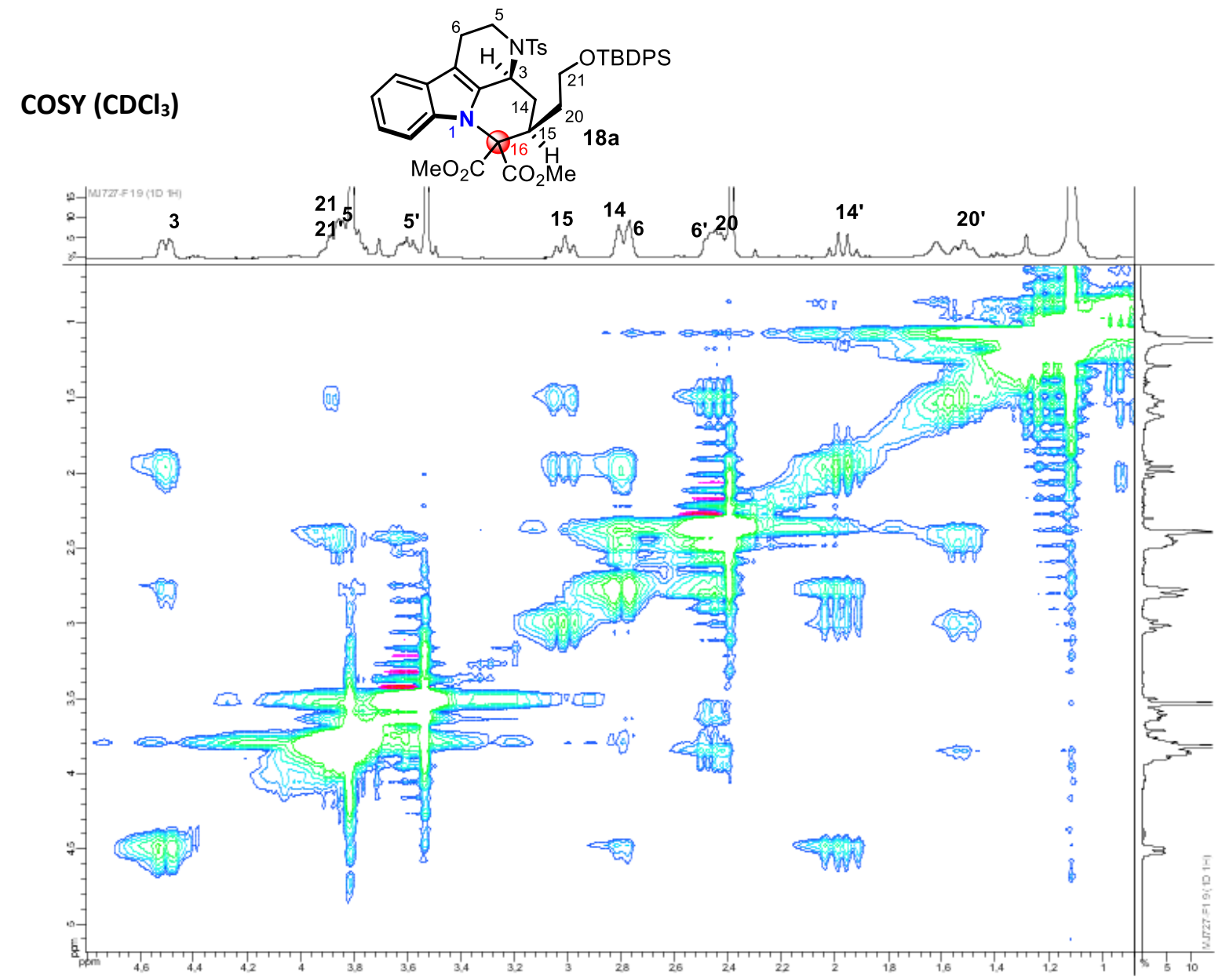

$\mathrm{HSQC}\left(\mathrm{CDCl}_{3}\right)$

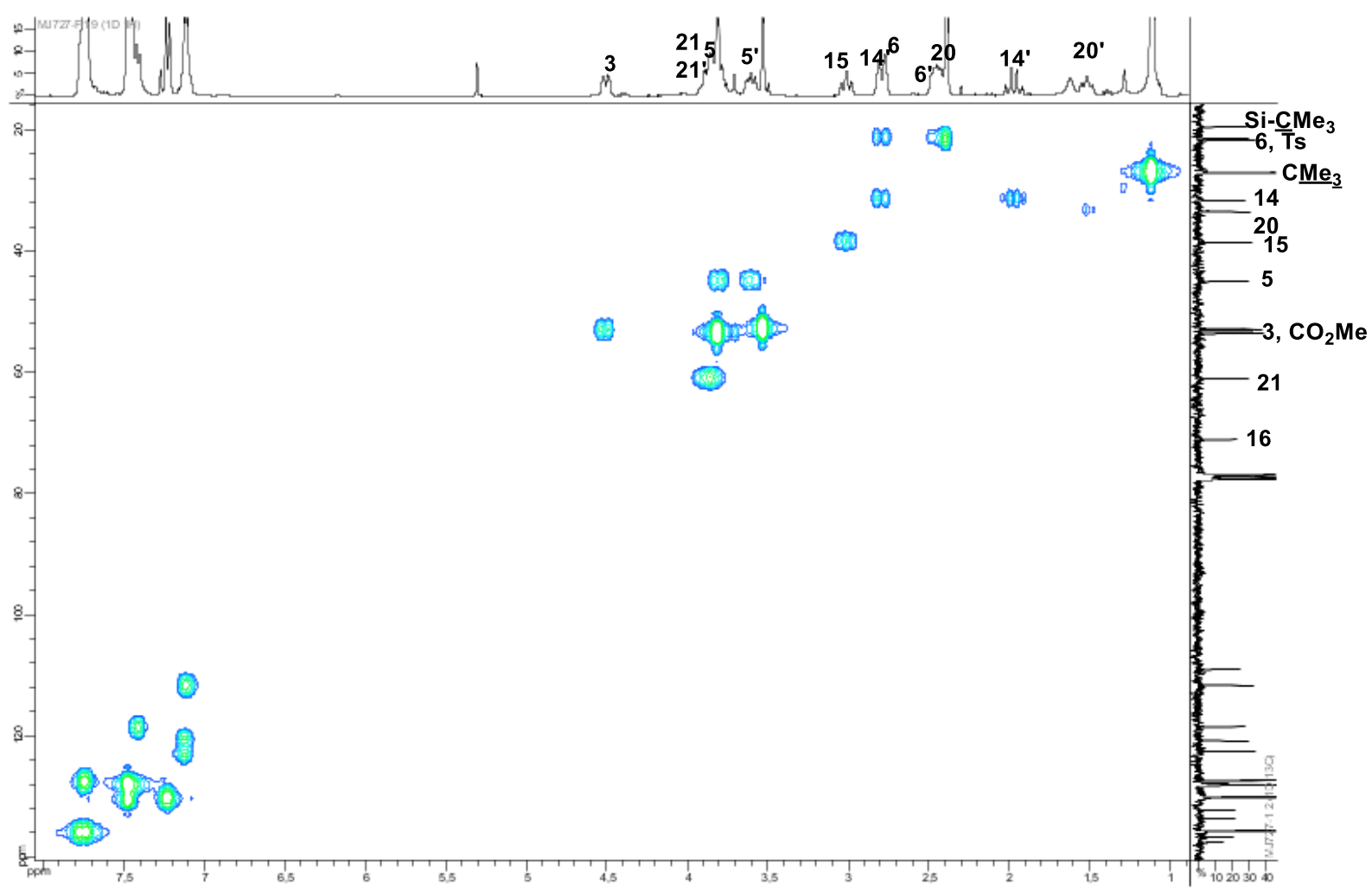

S23 
$\operatorname{NOESY}\left(\mathrm{CDCl}_{3}\right)$
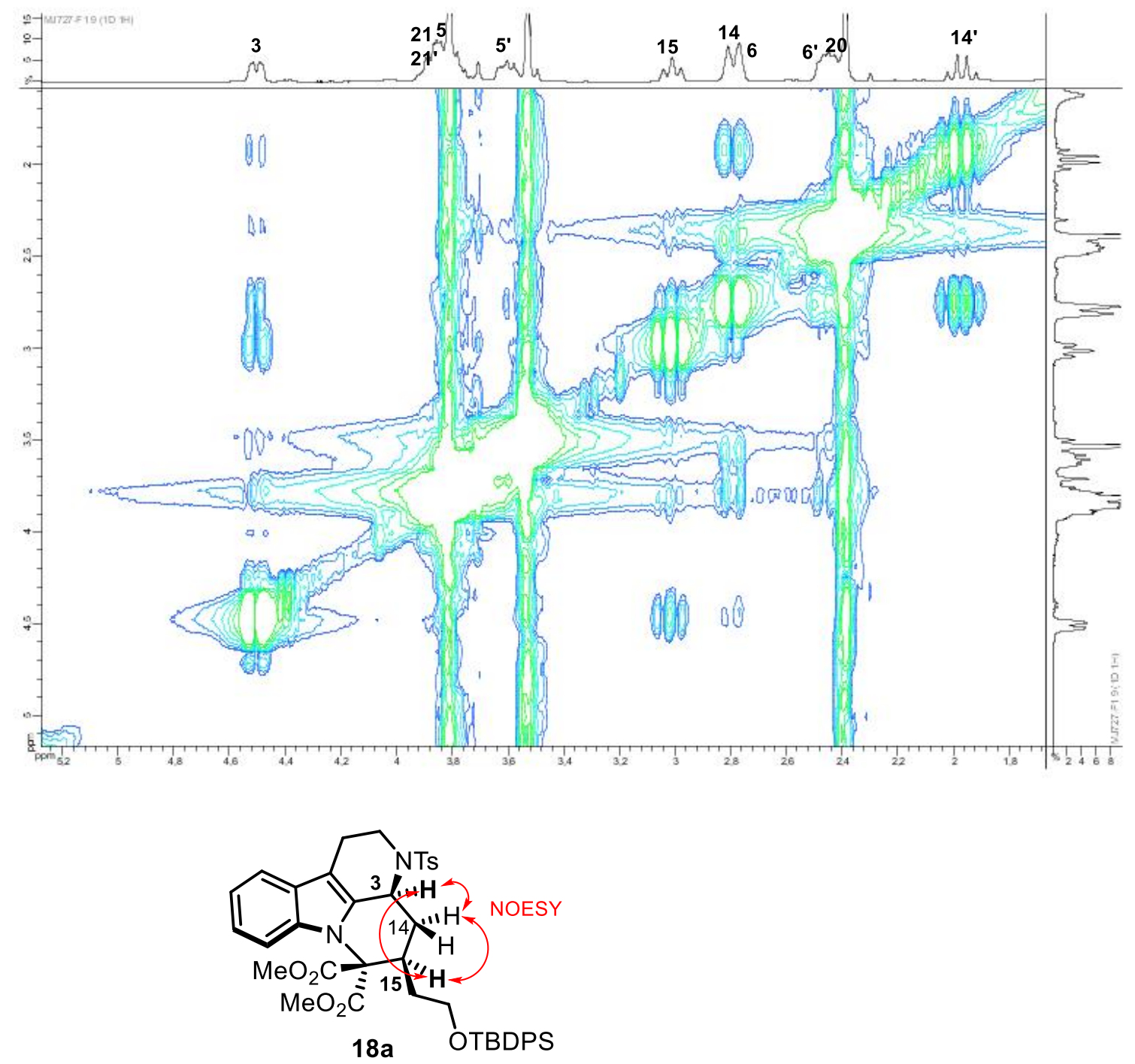
${ }^{1} \mathrm{H}$ NMR $\left(360 \mathrm{MHz}, \mathrm{CDCl}_{3}\right)$

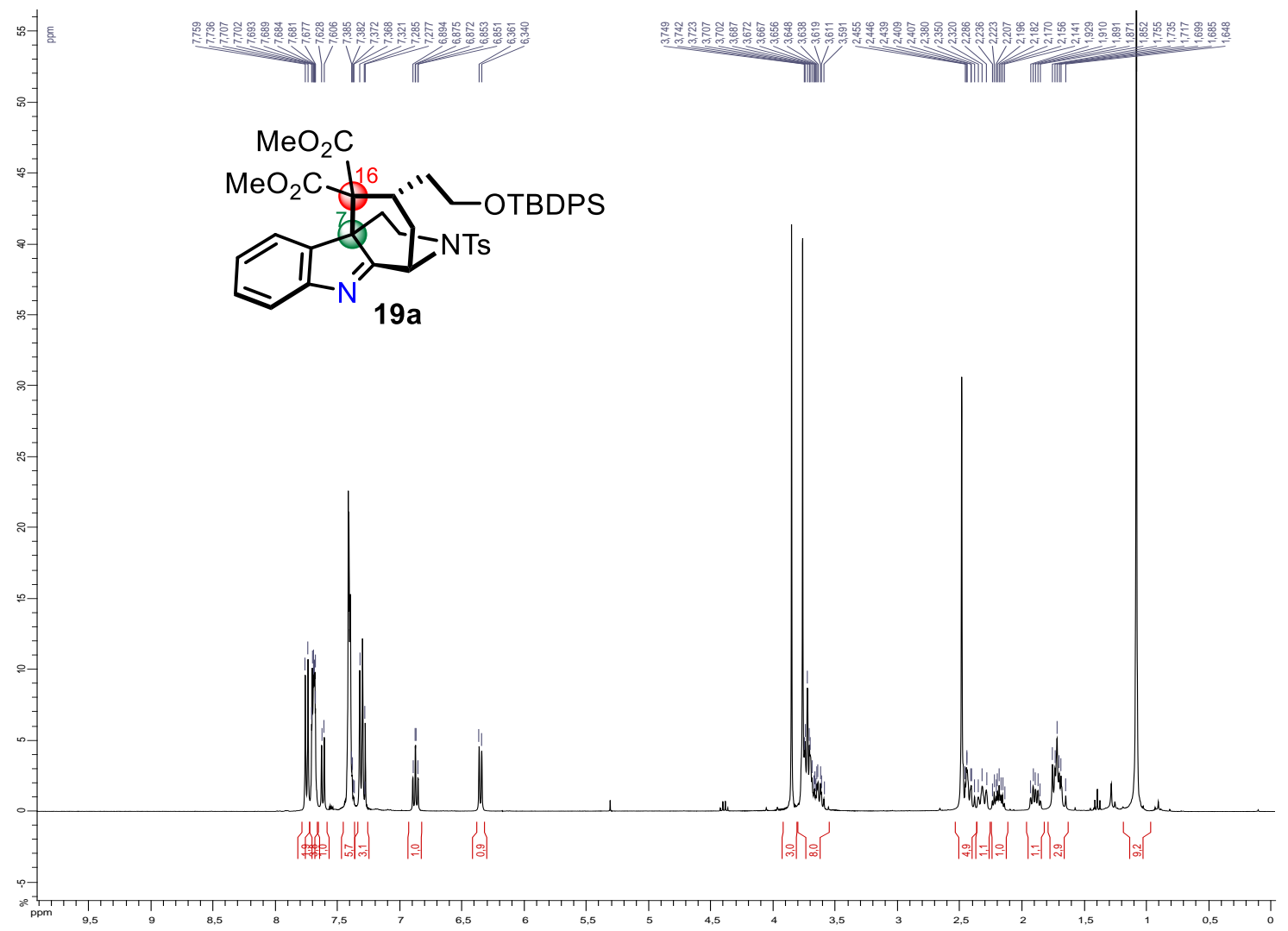

${ }^{13} \mathrm{C}$ NMR (91 MHz, $\left.\mathrm{CDCl}_{3}\right)$

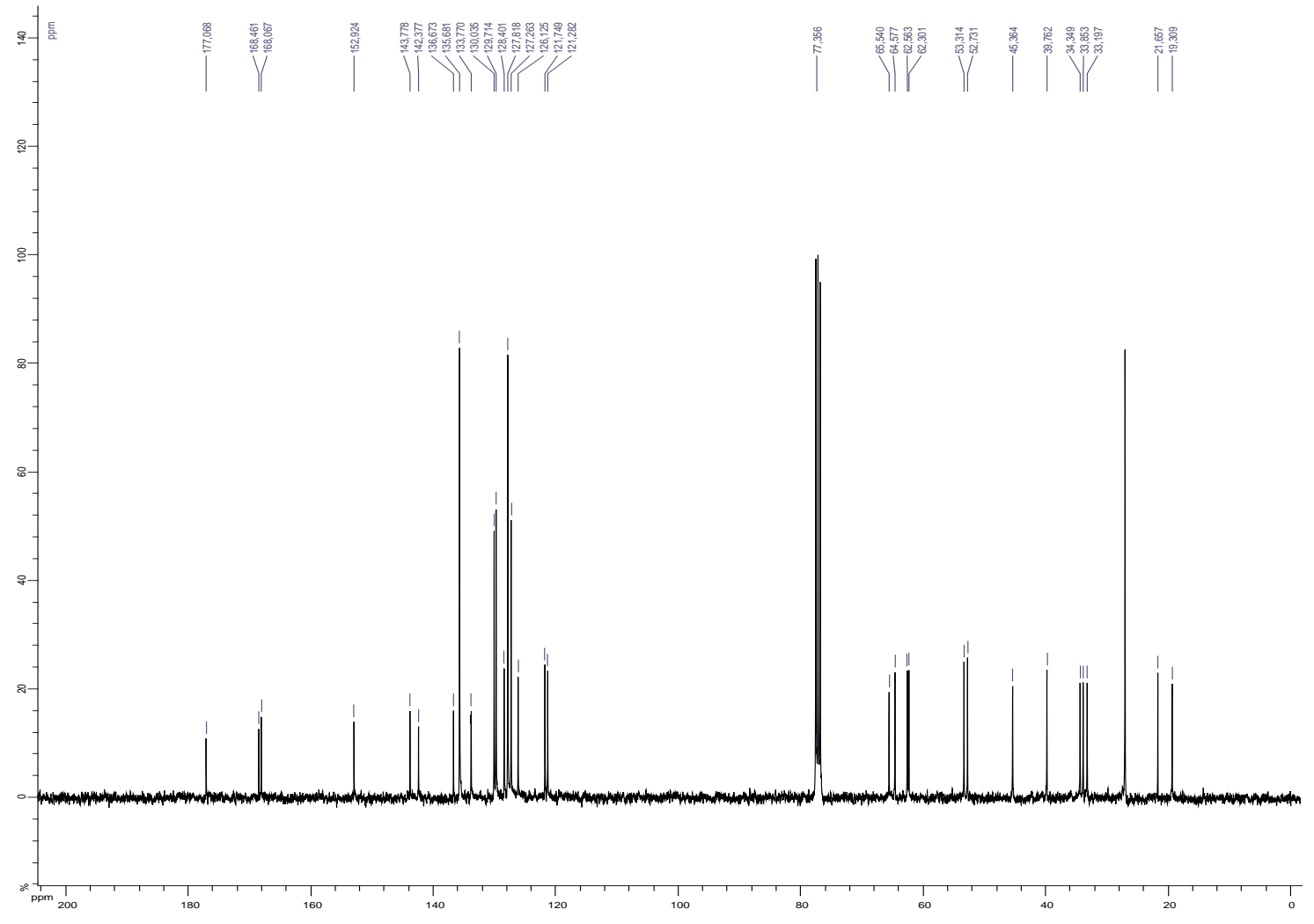




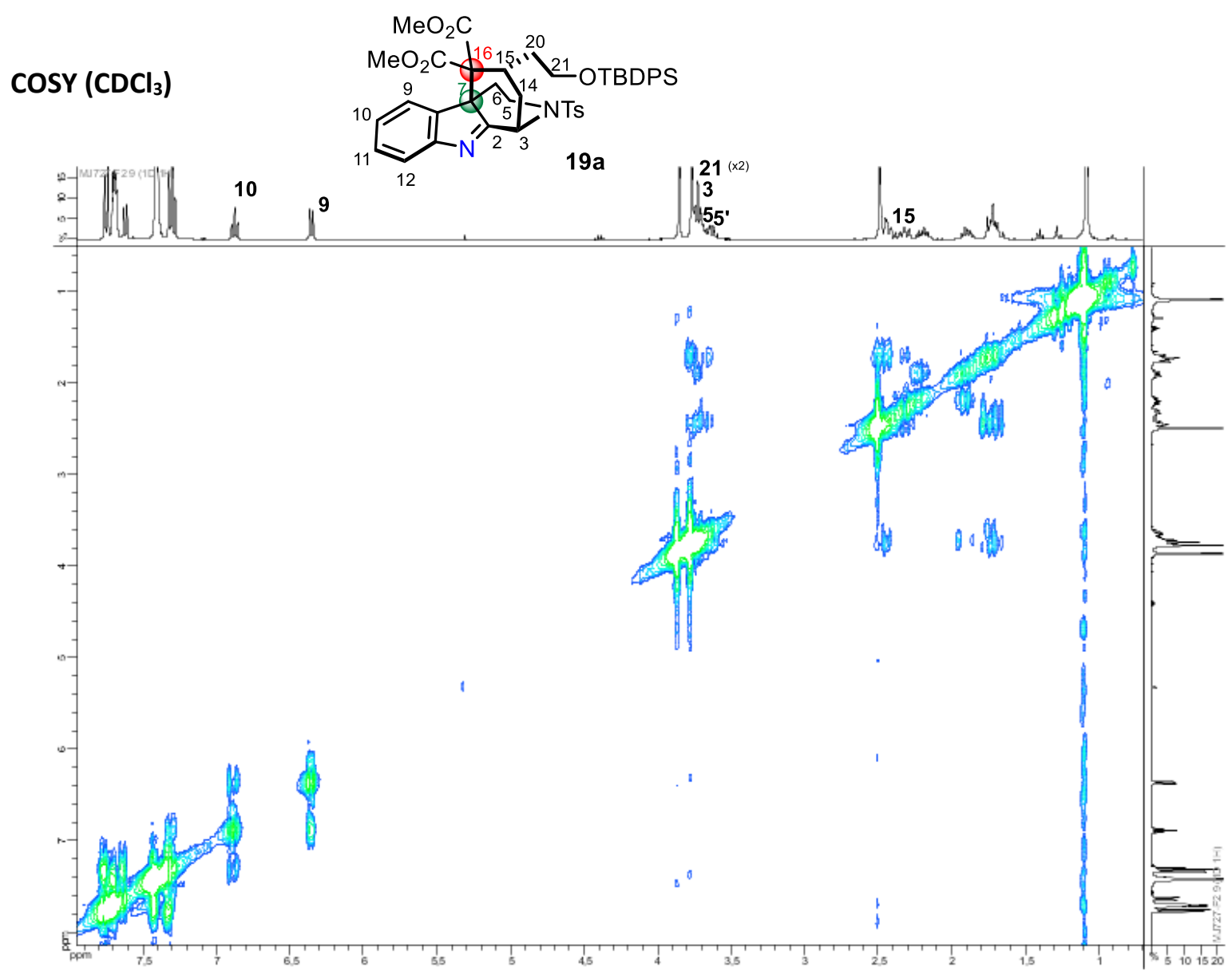

$\operatorname{HSQC}\left(\mathrm{CDCl}_{3}\right)$

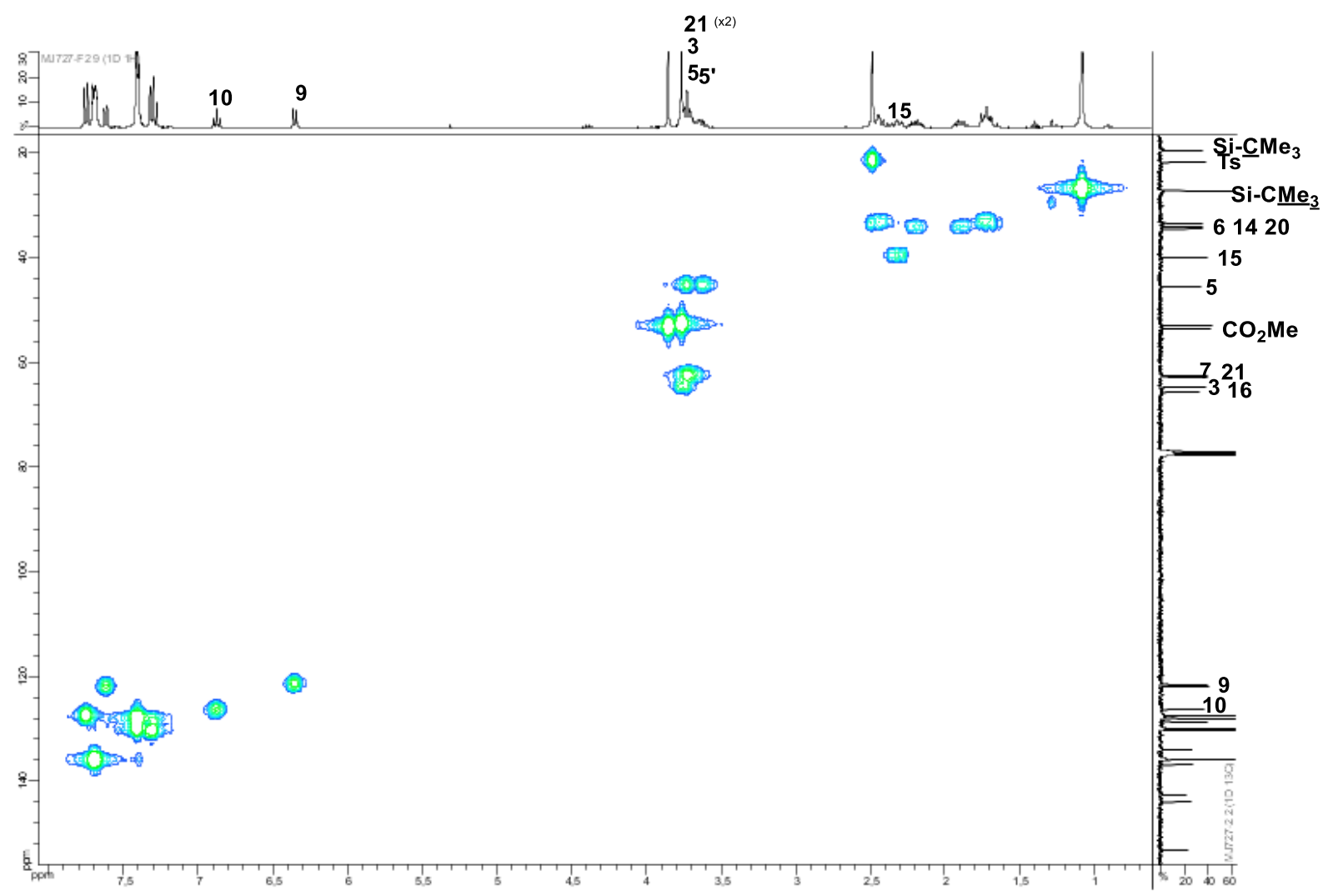

S26 
$\mathrm{HMBC}\left(\mathrm{CDCl}_{3}\right)$
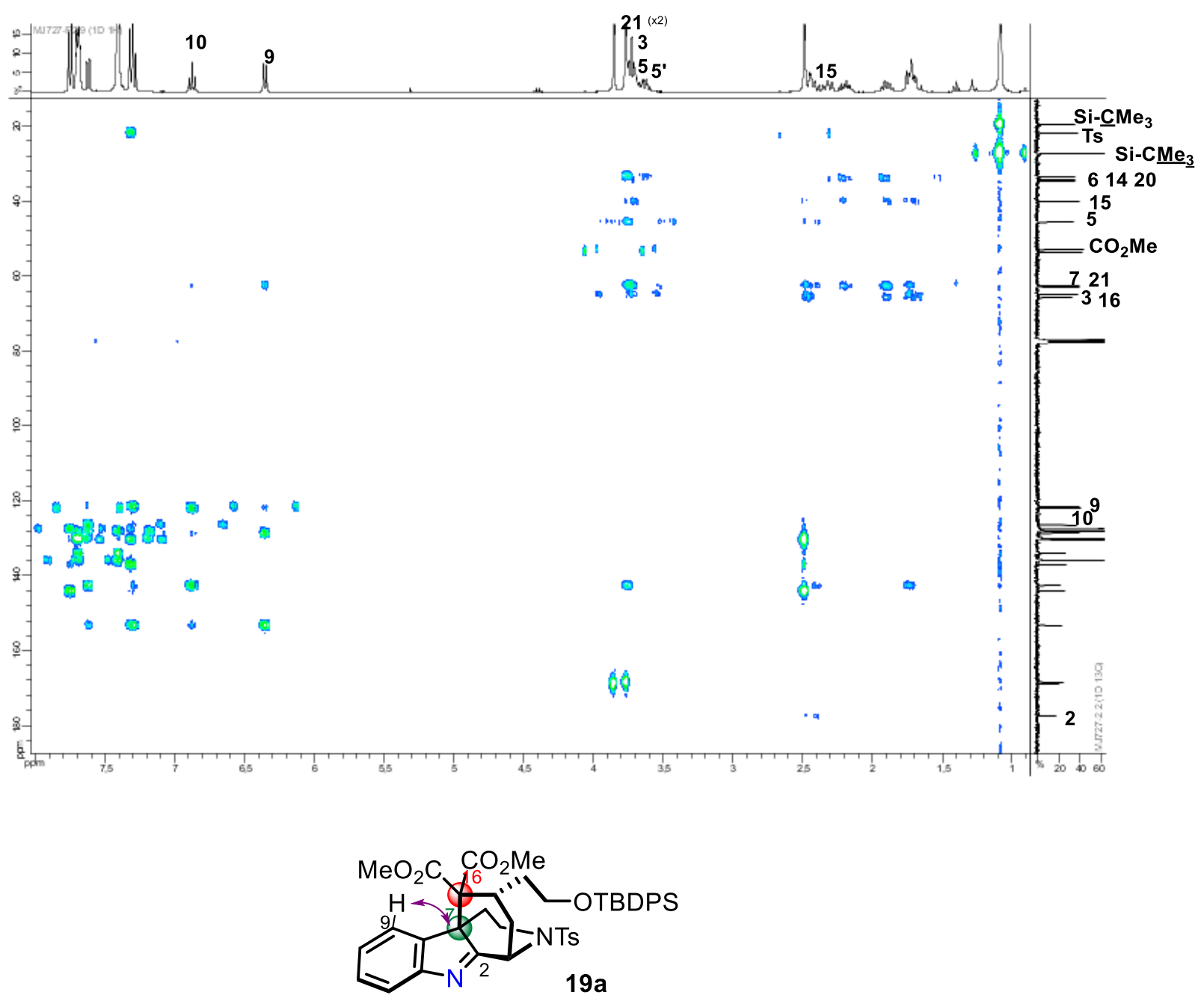

S27 
${ }^{1} \mathrm{H}$ NMR $\left(360 \mathrm{MHz}, \mathrm{CDCl}_{3}\right)$

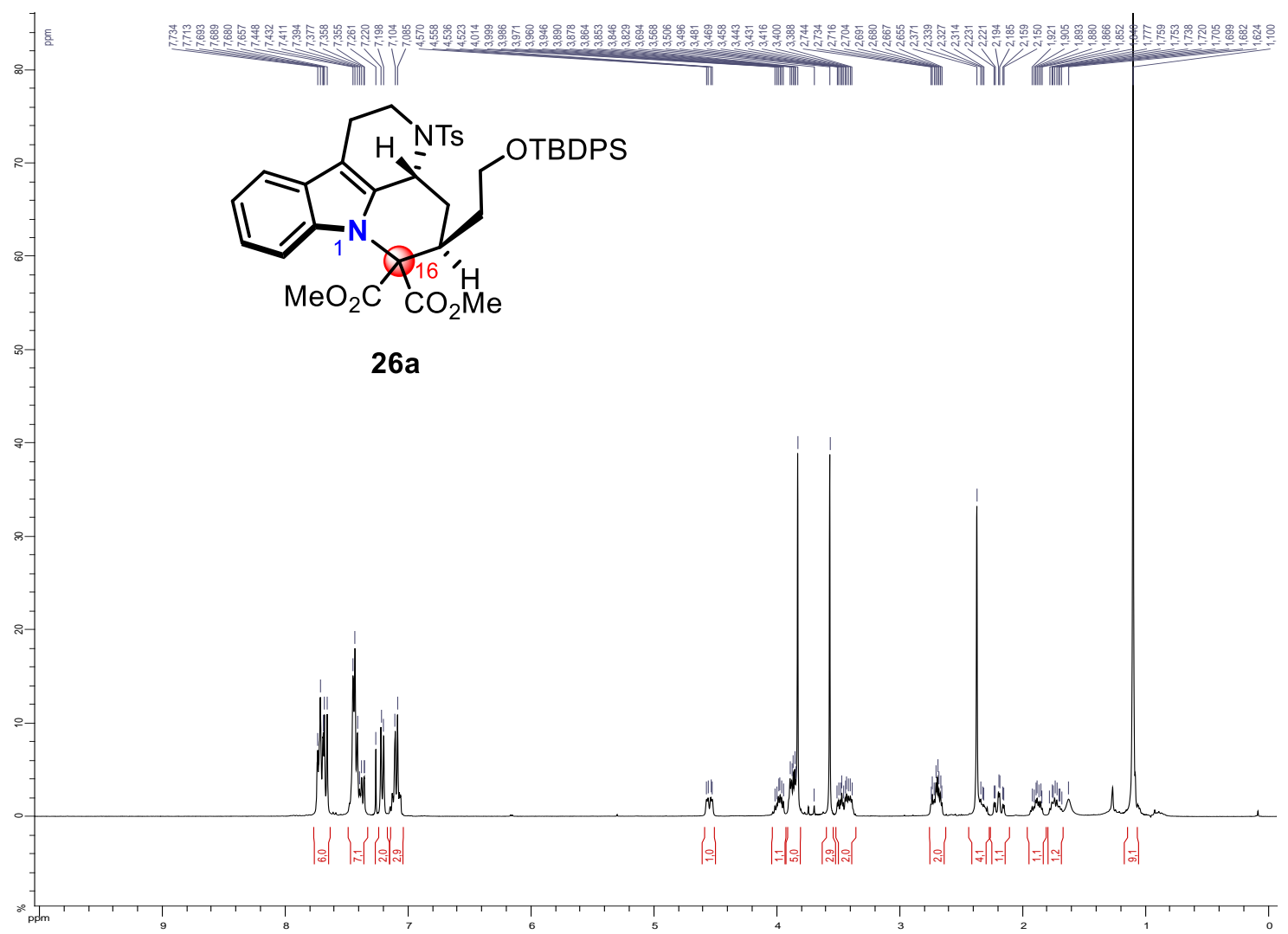

${ }^{13} \mathrm{C}$ NMR (91 MHz, $\left.\mathrm{CDCl}_{3}\right)$

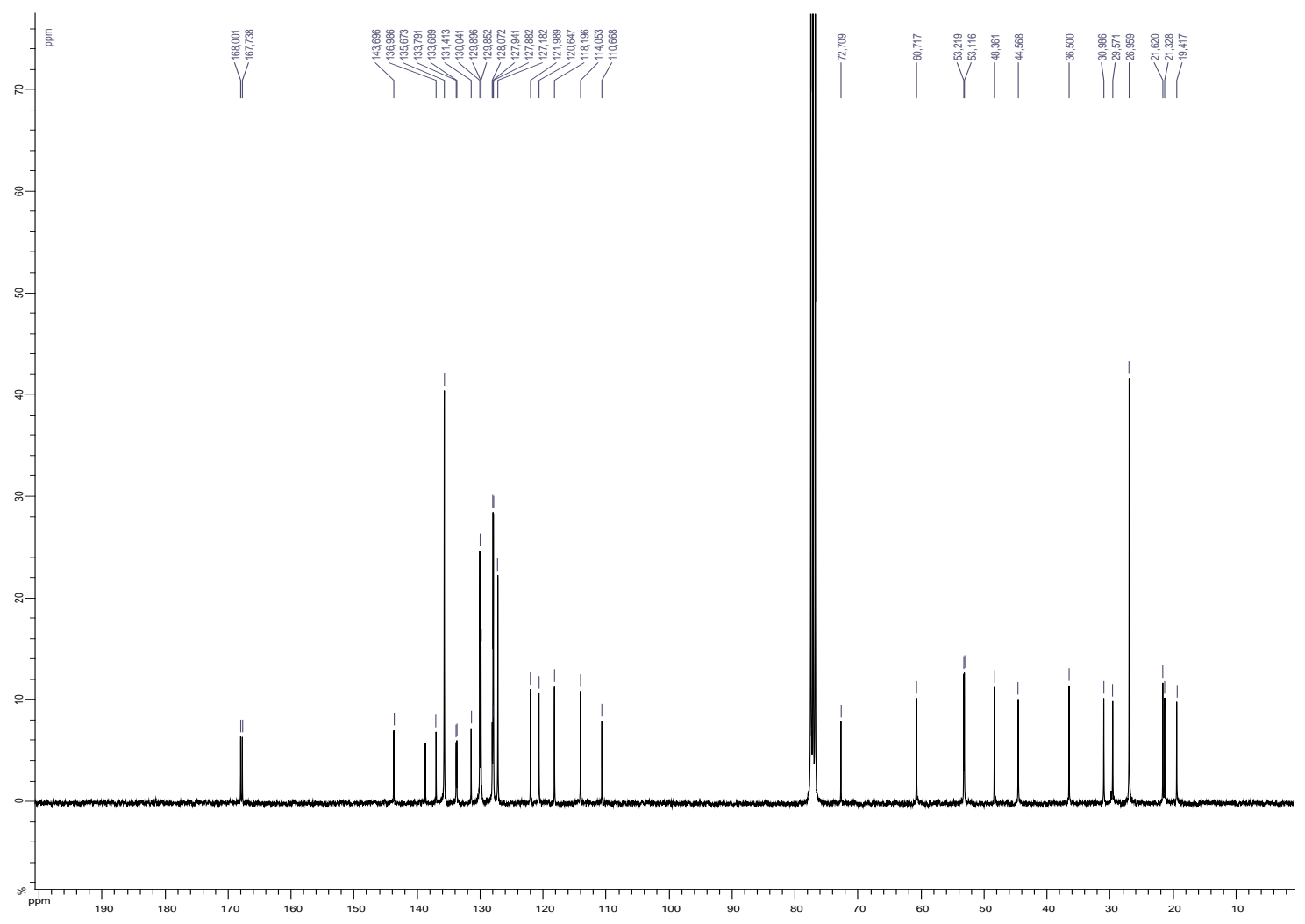




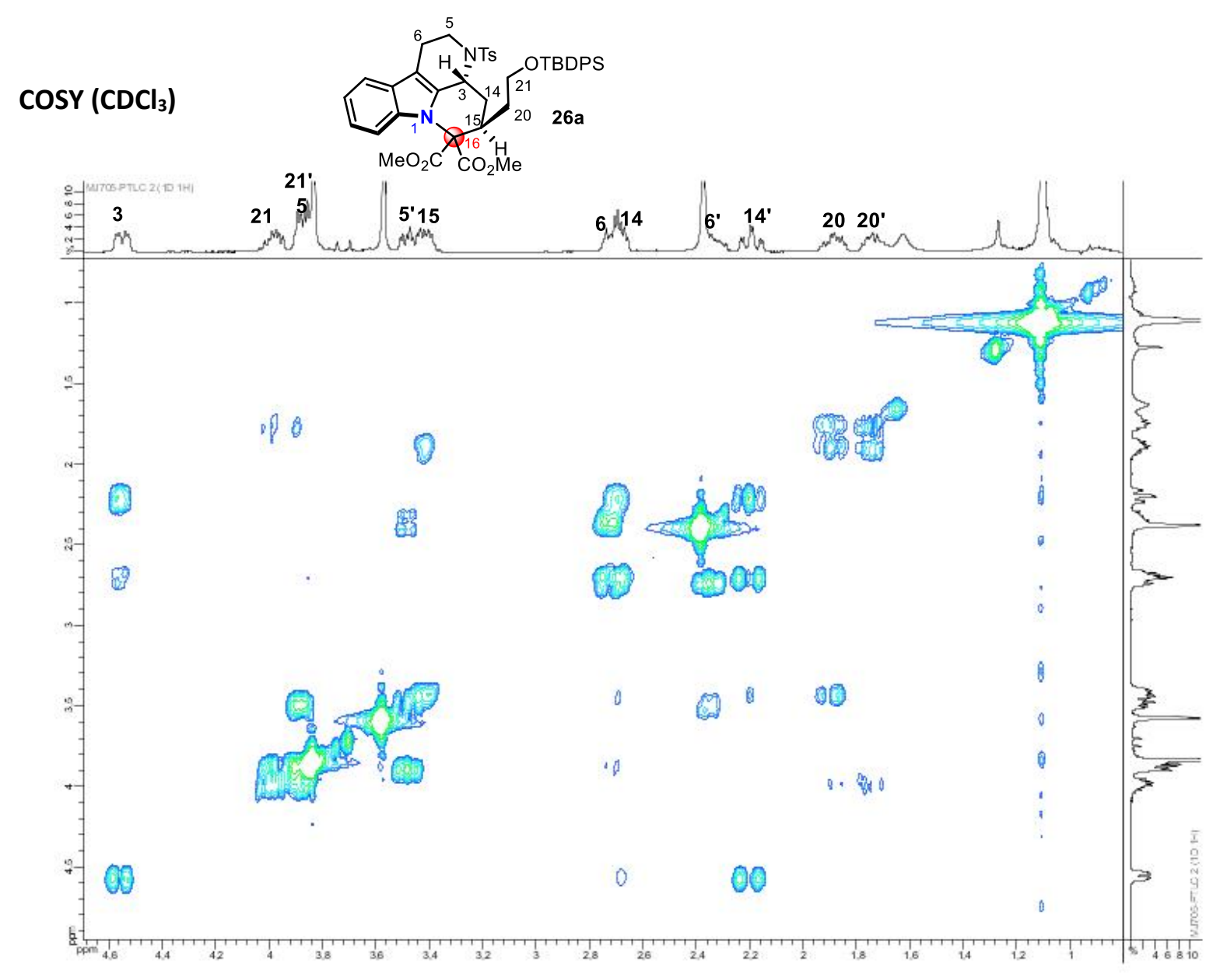

$\mathrm{HSQC}\left(\mathrm{CDCl}_{3}\right)$

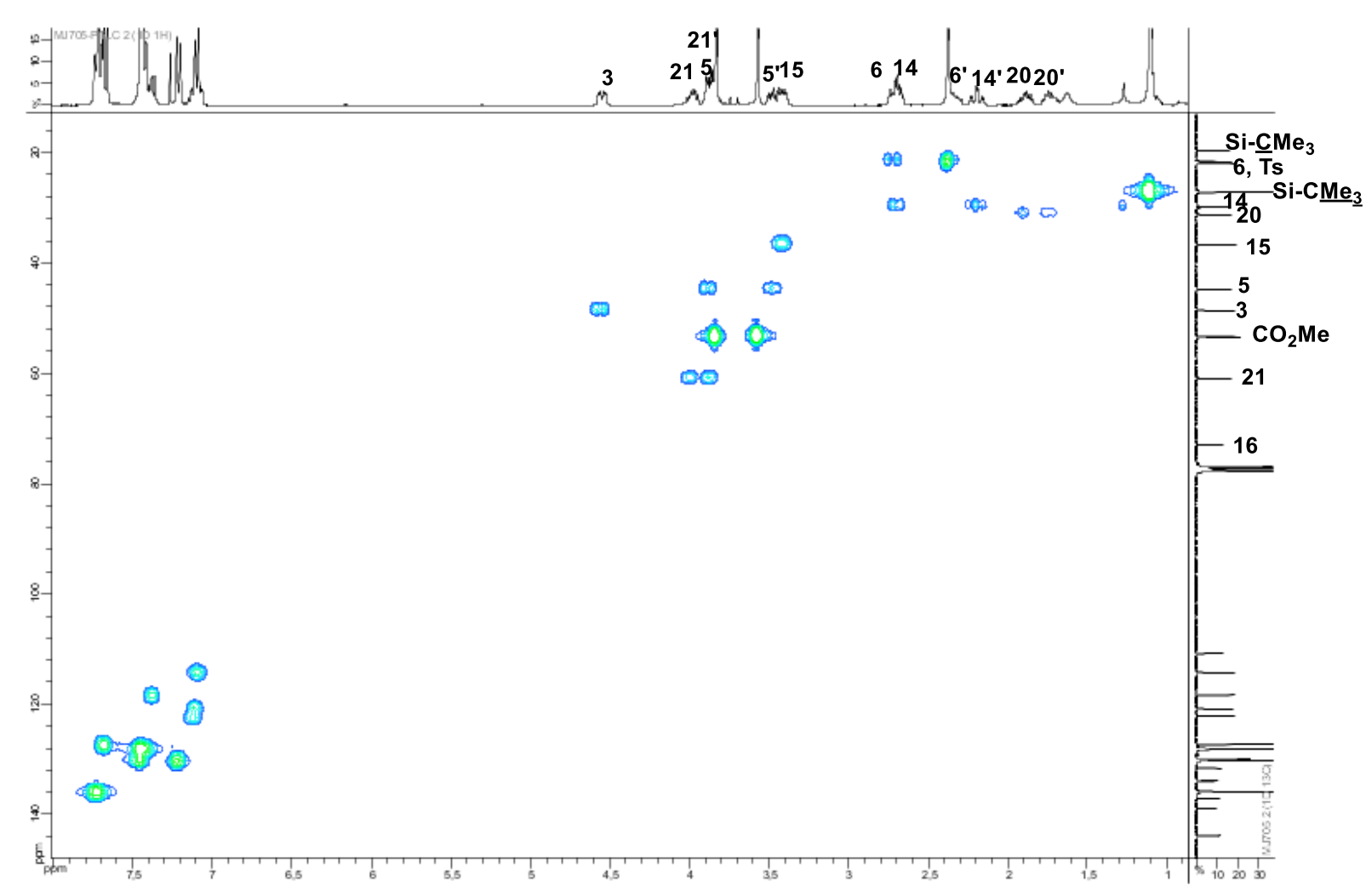


$\operatorname{NOESY}\left(\mathrm{CDCl}_{3}\right)$
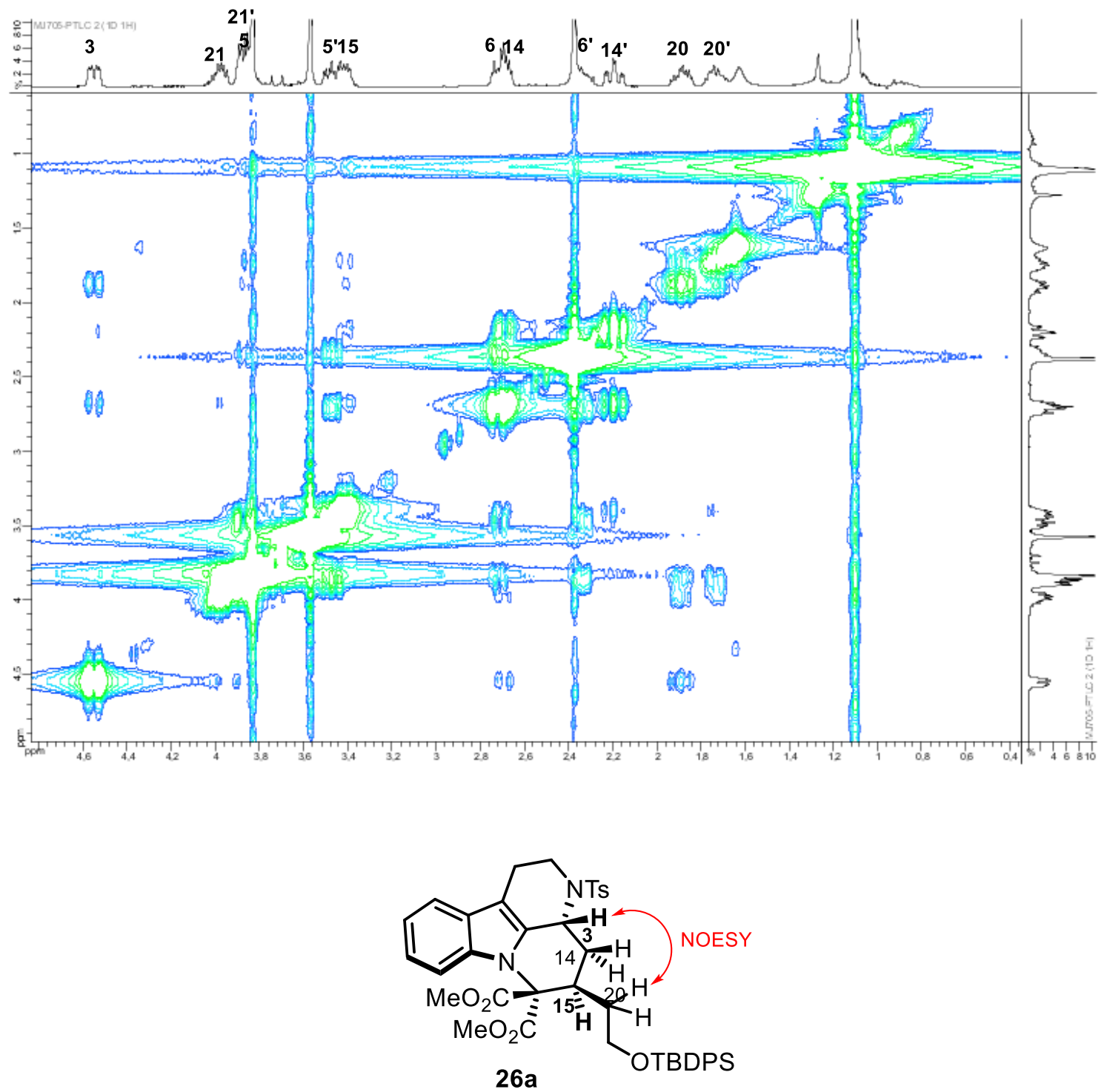
${ }^{1} \mathrm{H}$ NMR $\left(300 \mathrm{MHz}, \mathrm{CDCl}_{3}\right)$

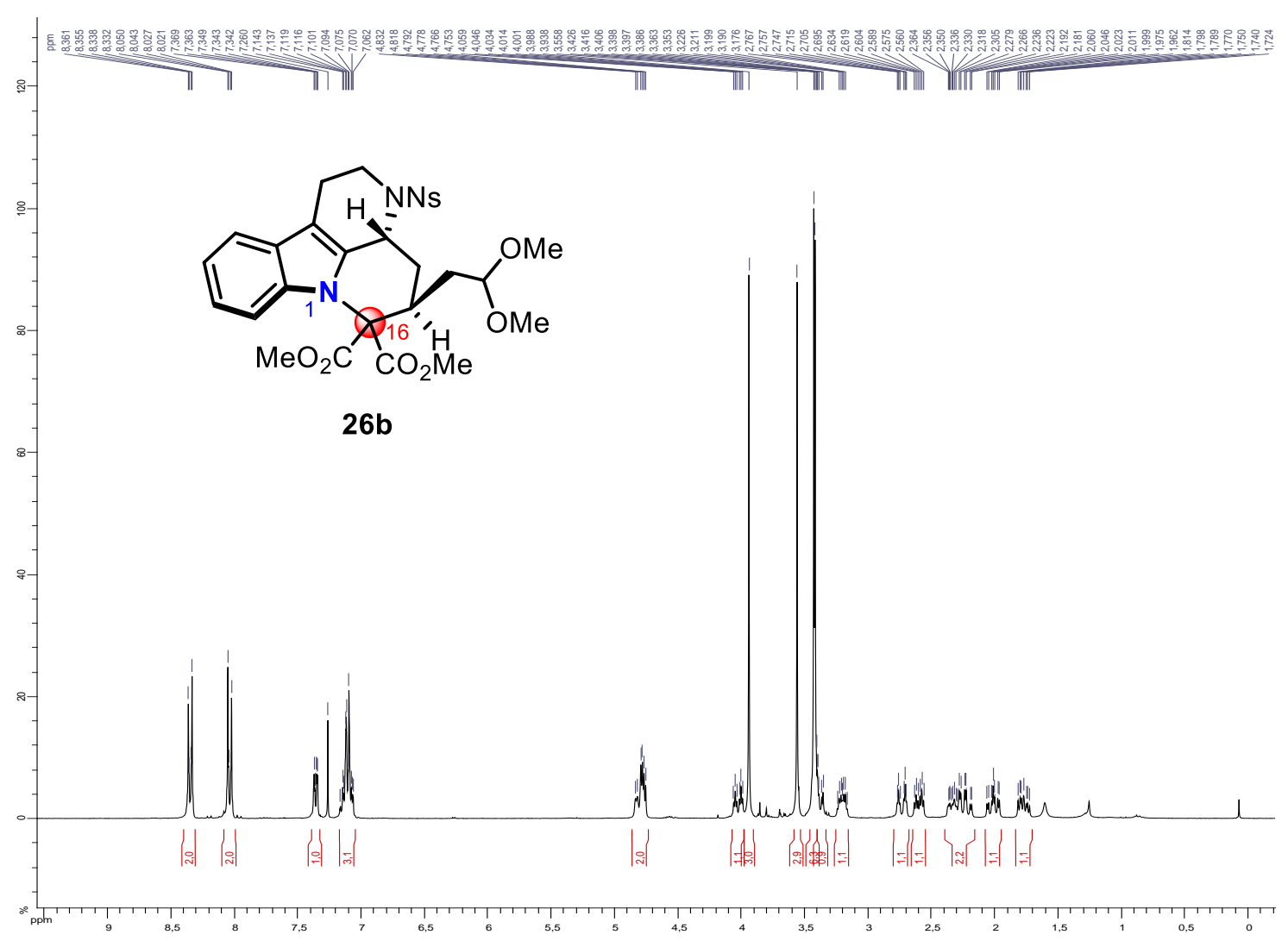

${ }^{13} \mathrm{C}$ NMR $\left(75 \mathrm{MHz}, \mathrm{CDCl}_{3}\right)$

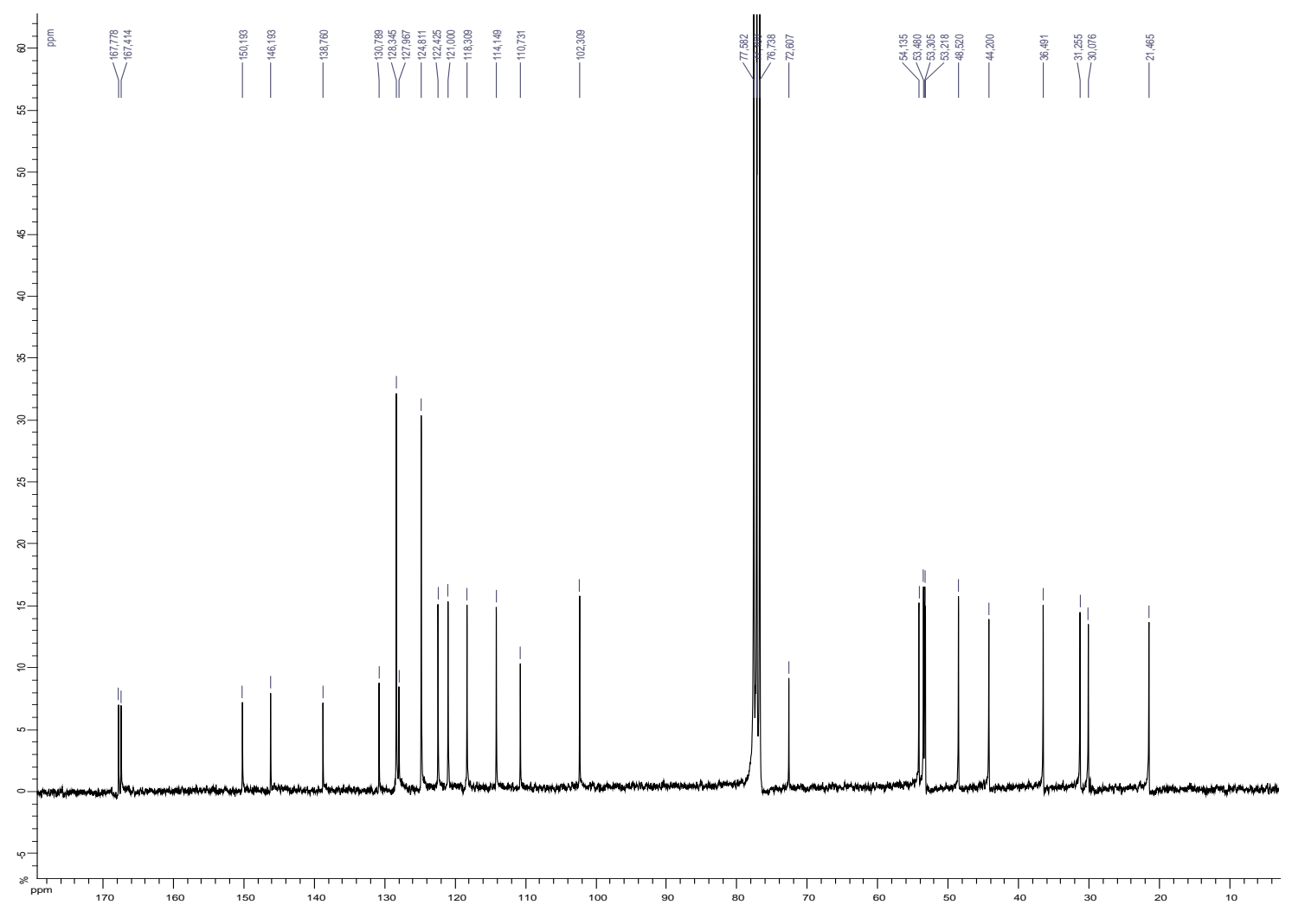


$\operatorname{cosY}\left(\mathrm{CDCl}_{3}\right)$
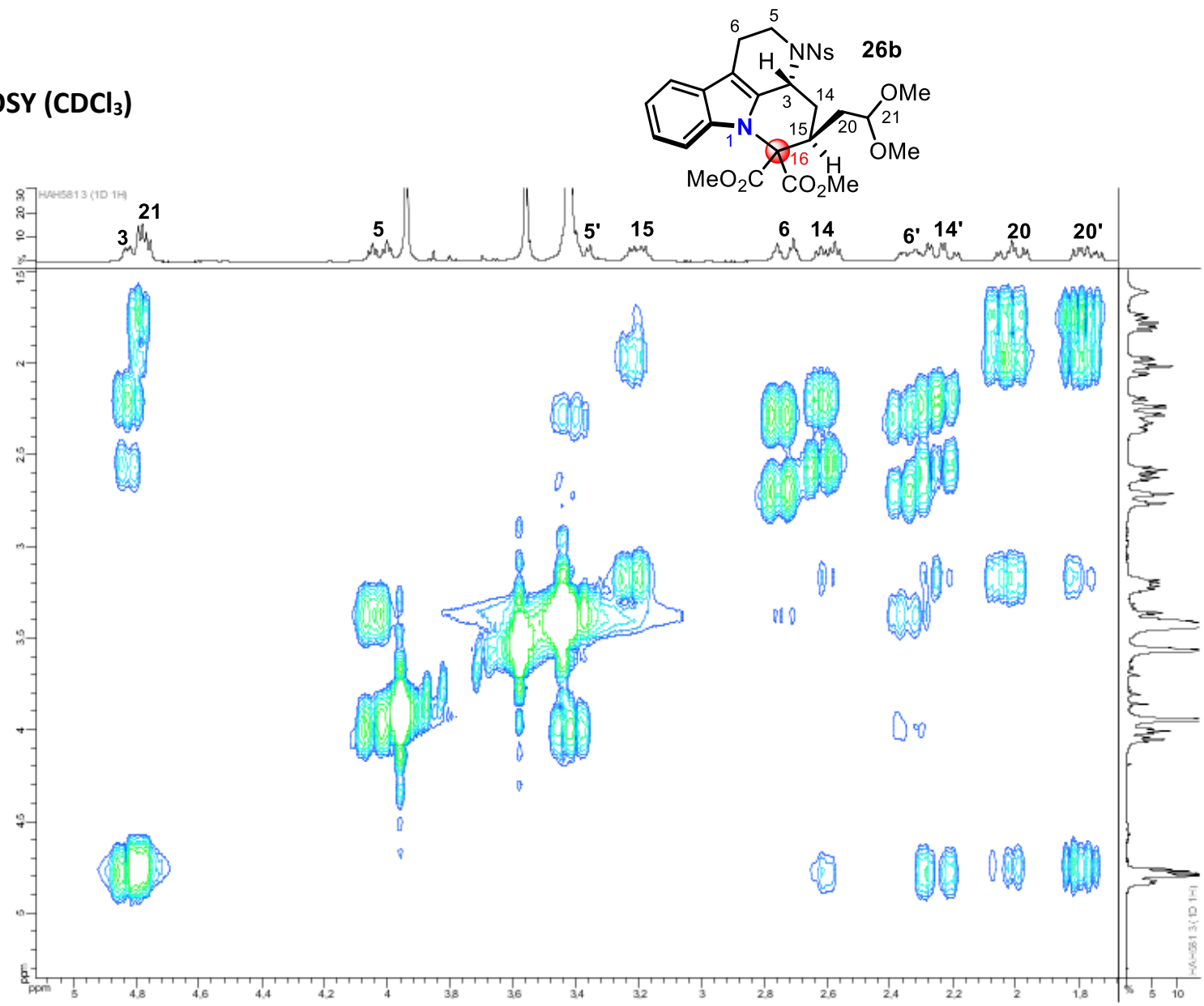

$\mathrm{HSQC}\left(\mathrm{CDCl}_{3}\right)$

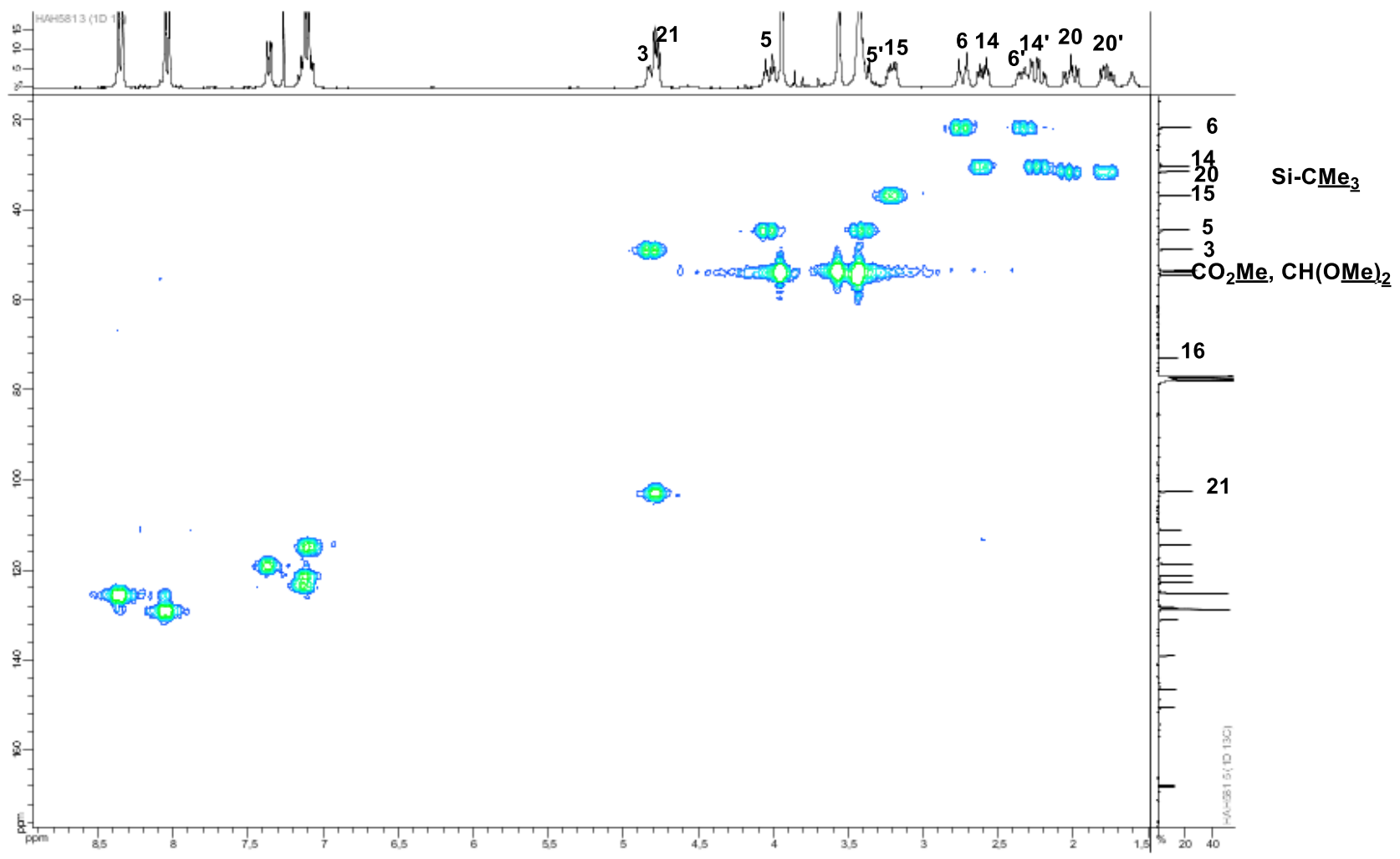

S32 
$\operatorname{NOESY}\left(\mathrm{CDCl}_{3}\right)$
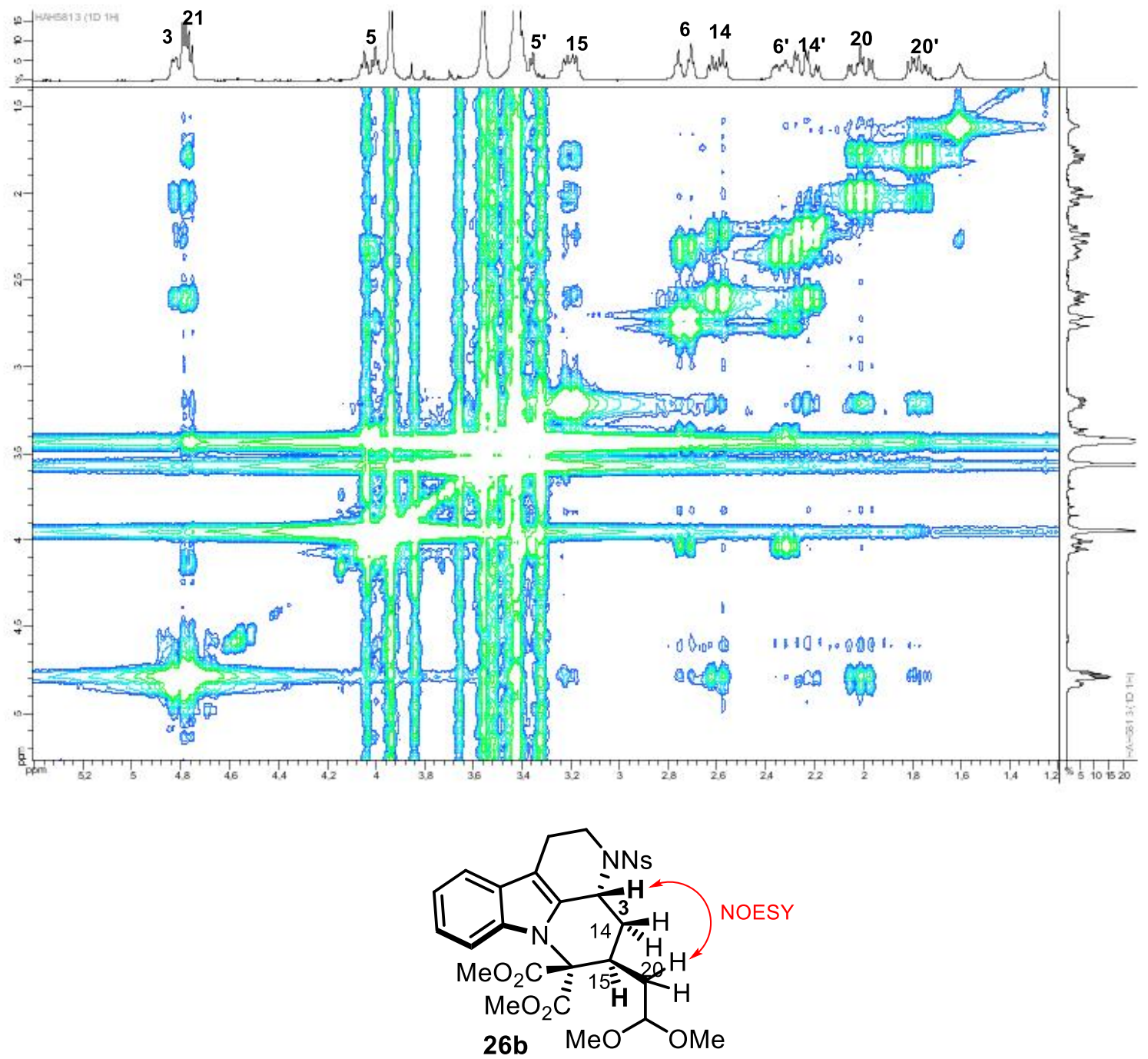
${ }^{1} \mathrm{H}$ NMR $\left(360 \mathrm{MHz}, \mathrm{CDCl}_{3}\right)$

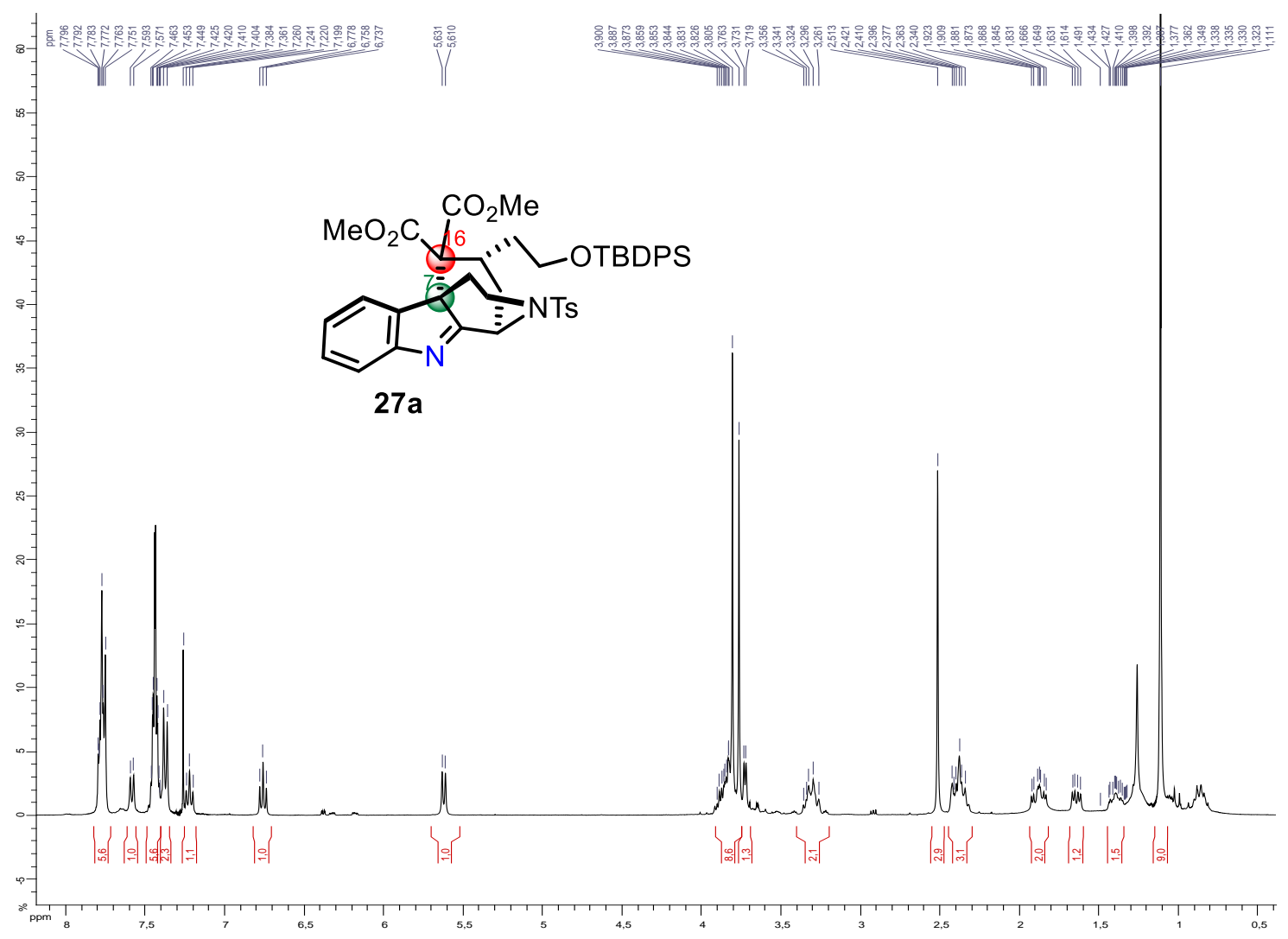

${ }^{13} \mathrm{C}$ NMR (100 MHz, $\left.\mathrm{CDCl}_{3}\right)$

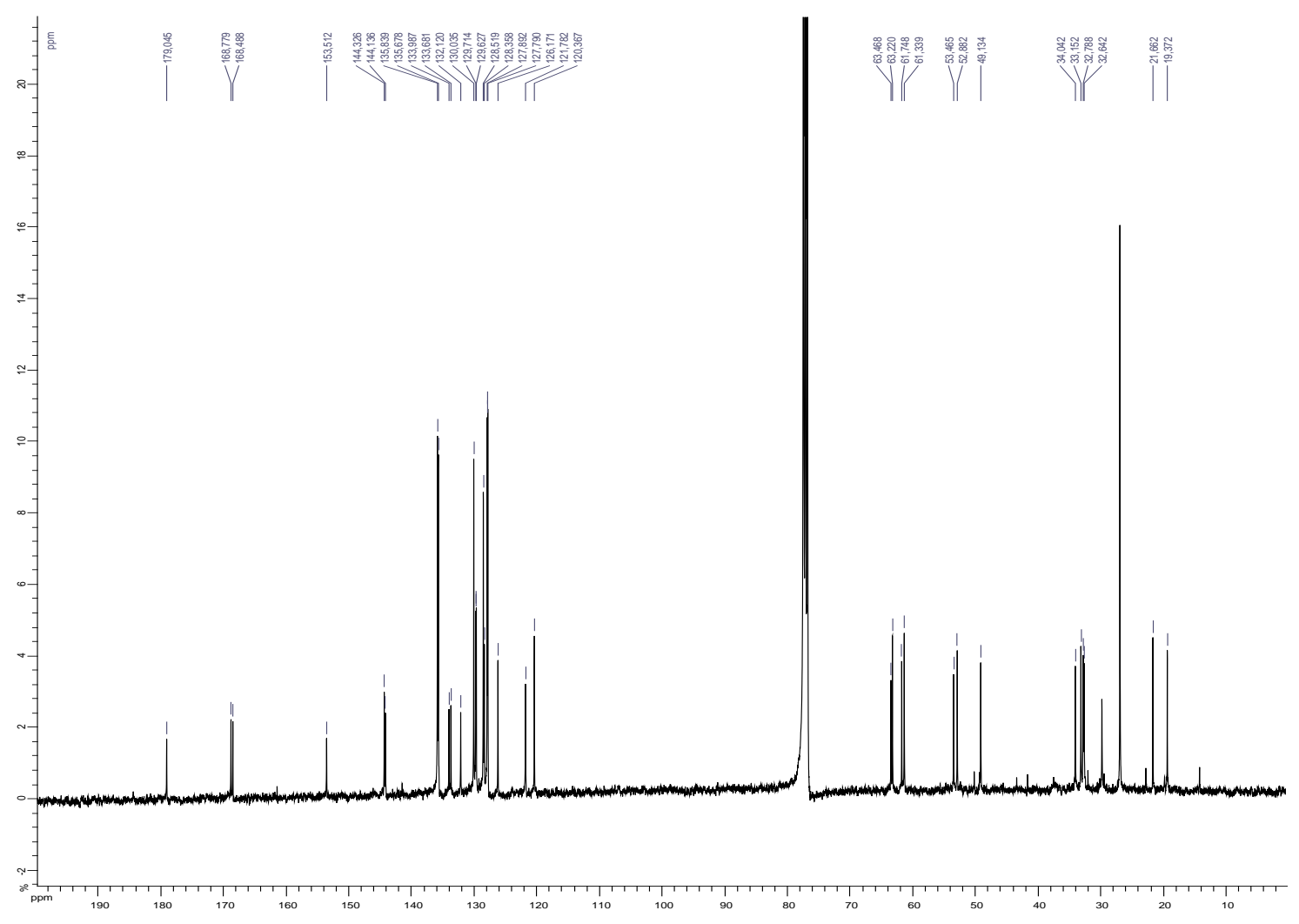




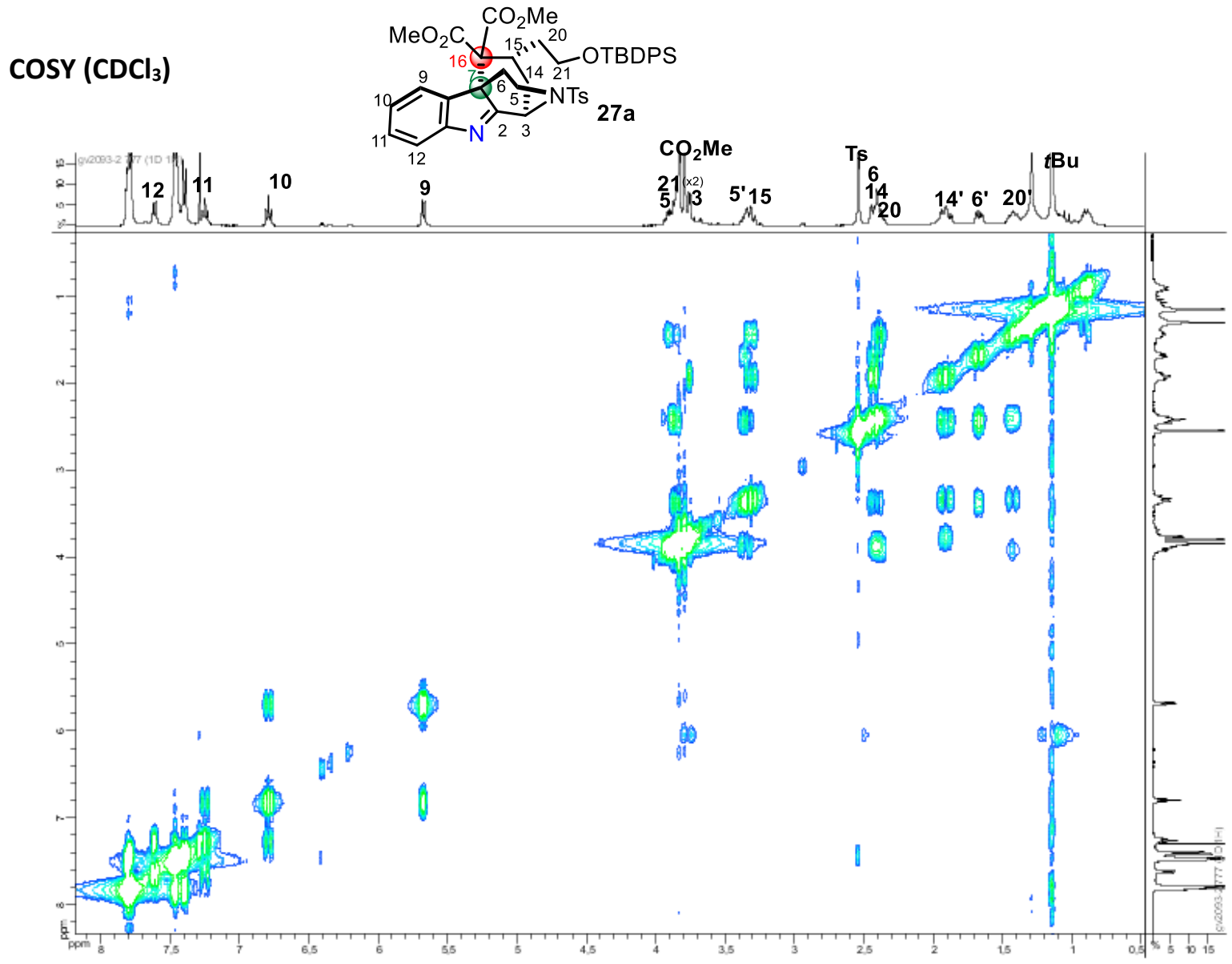

$\mathrm{HSQC}\left(\mathrm{CDCl}_{3}\right)$

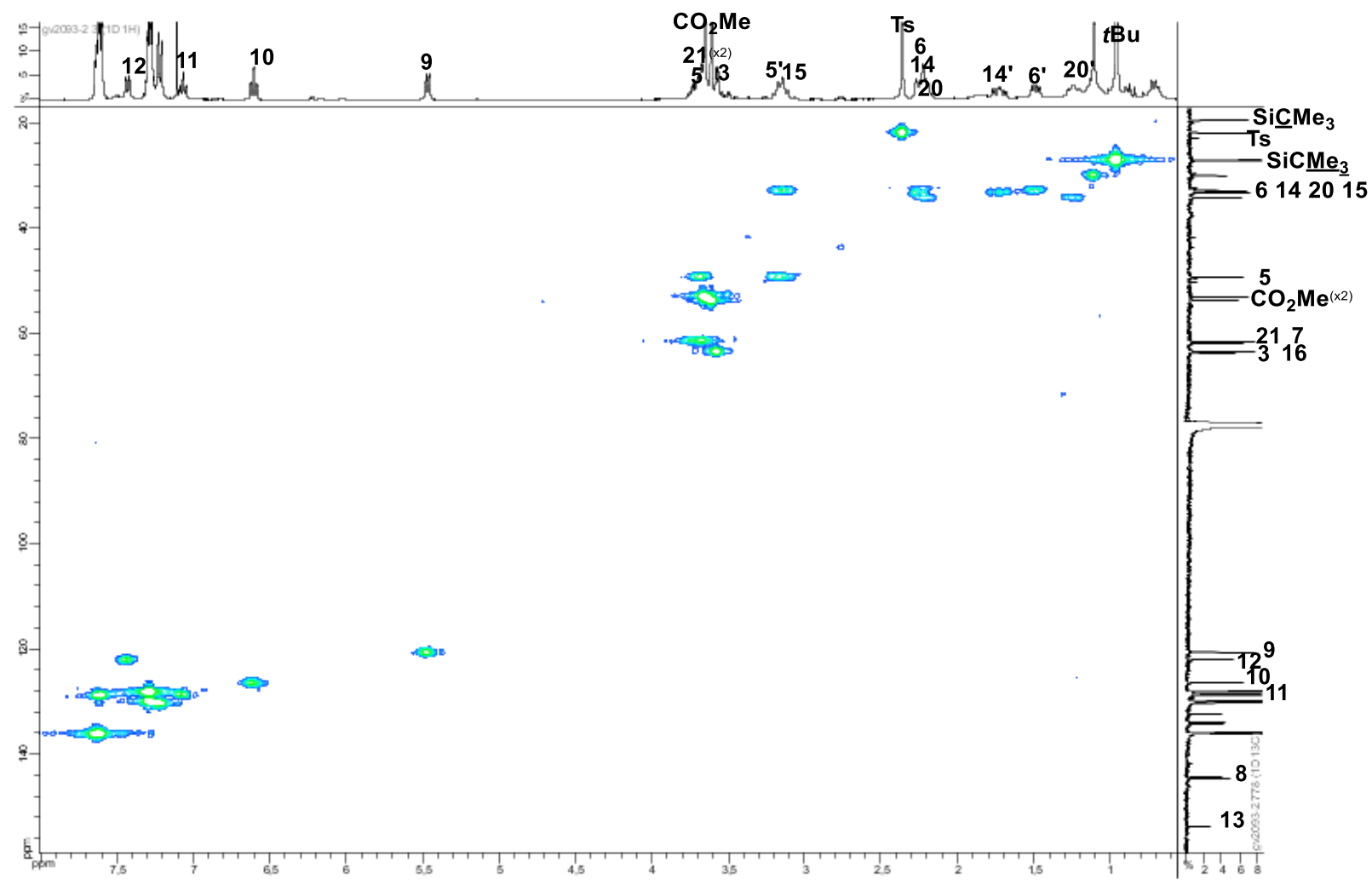

S35 


\section{$\mathrm{HMBC}\left(\mathrm{CDCl}_{3}\right)$}

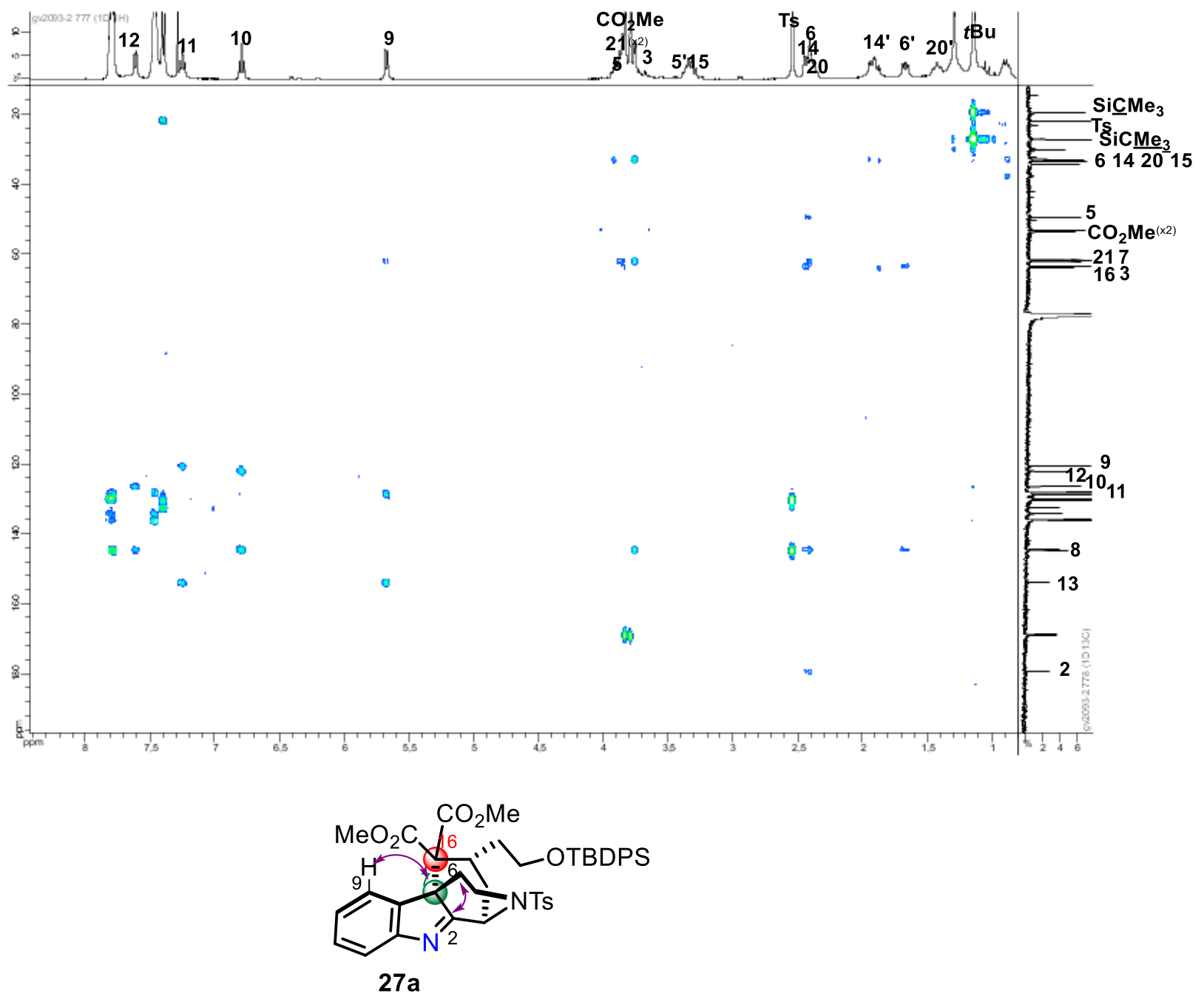


${ }^{1} \mathrm{H}$ NMR $\left(300 \mathrm{MHz}, \mathrm{CDCl}_{3}\right)$

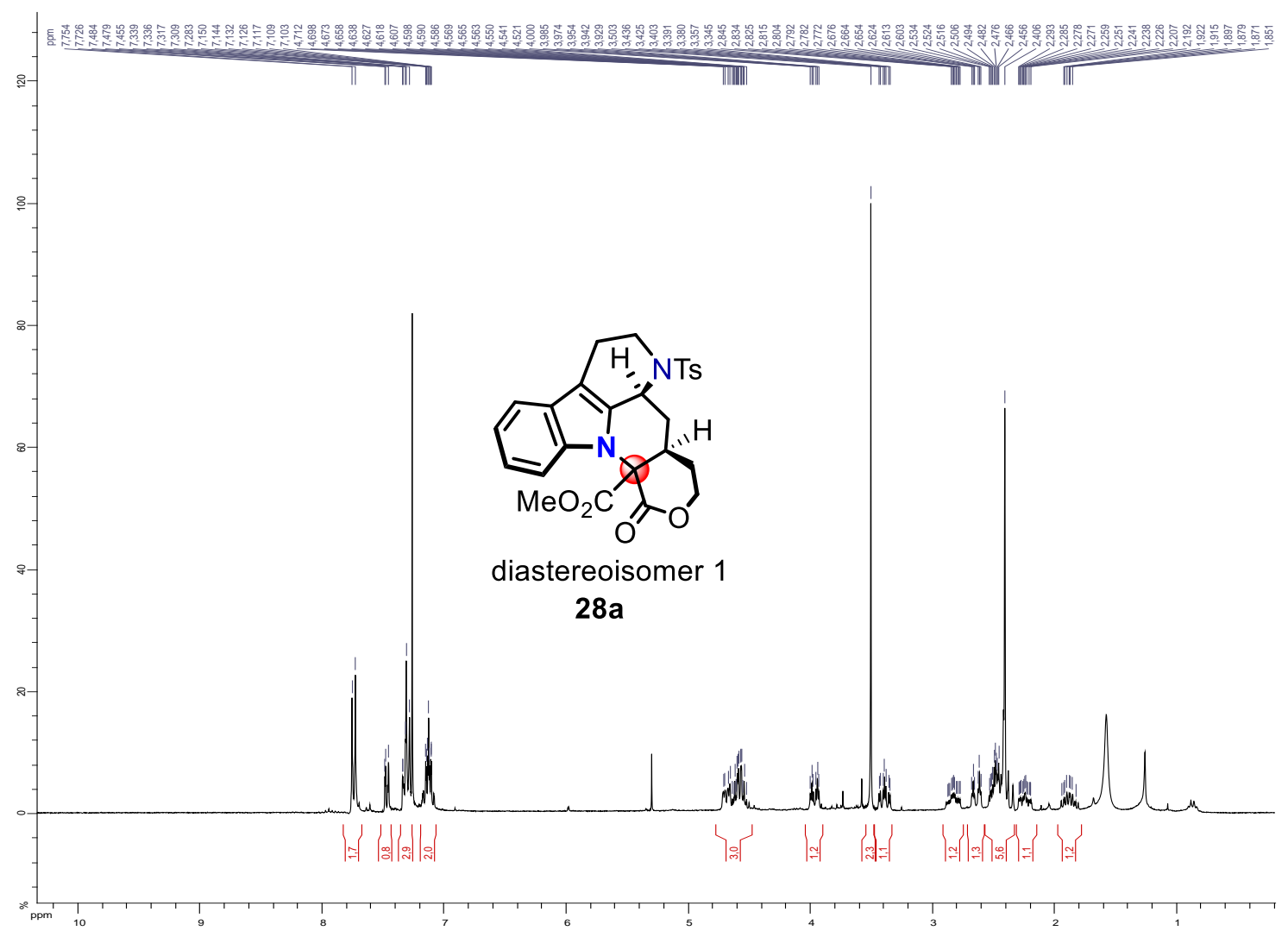

${ }^{13} \mathrm{C}$ NMR $\left(100 \mathrm{MHz}, \mathrm{CDCl}_{3}\right)$

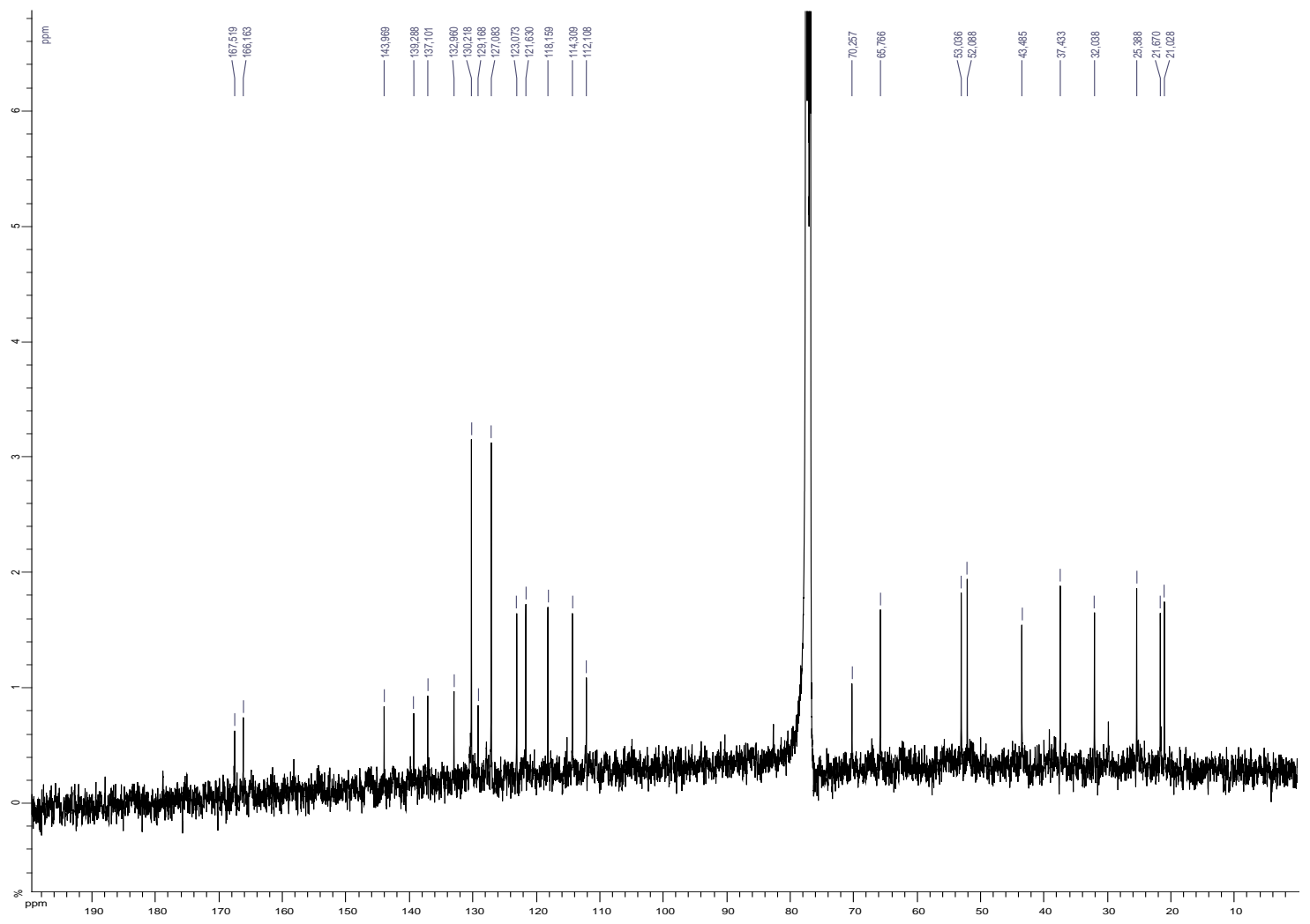


${ }^{1} \mathrm{H}$ NMR $\left(360 \mathrm{MHz}, \mathrm{CDCl}_{3}\right)$

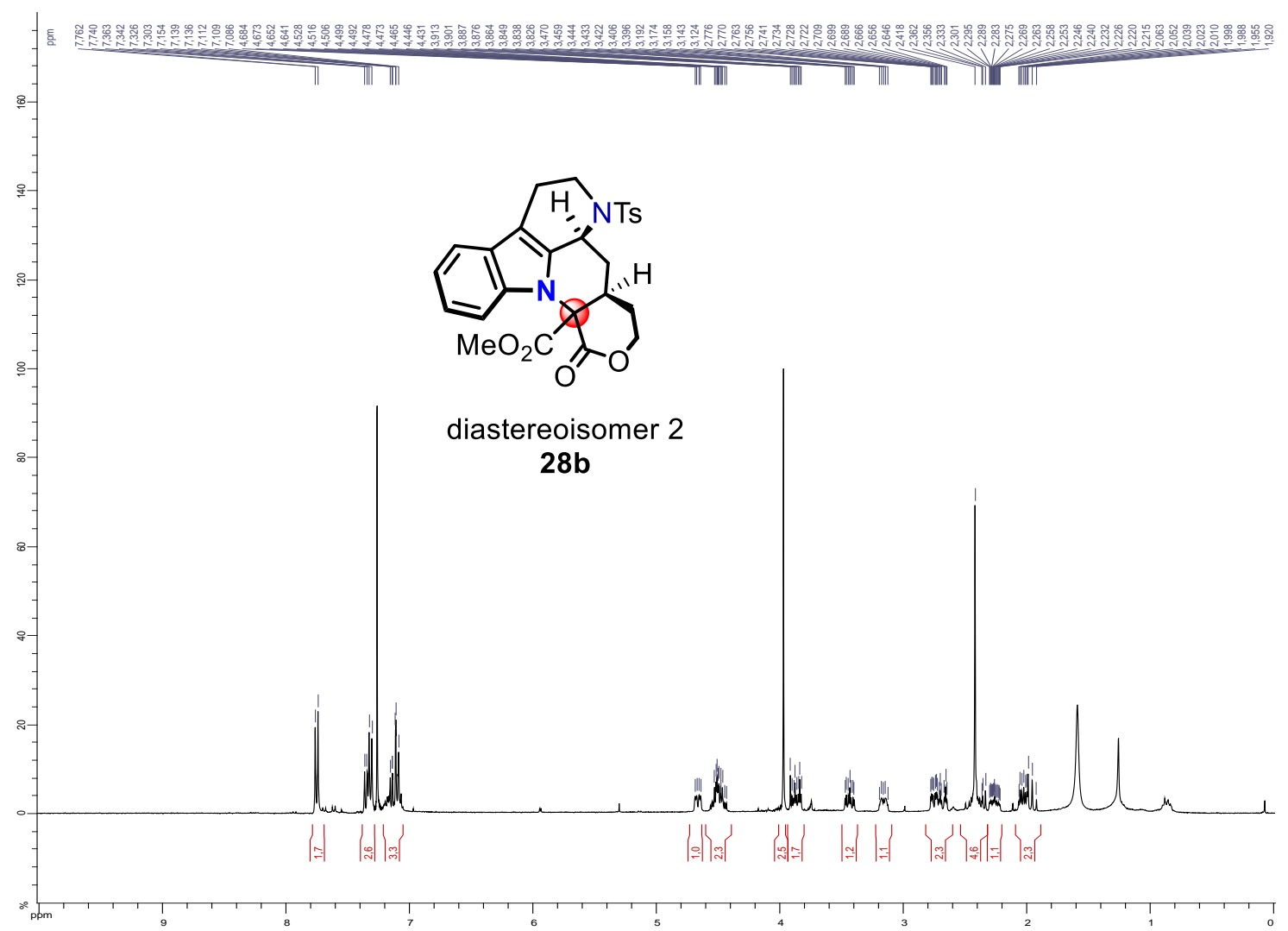

${ }^{13} \mathrm{C}$ NMR (100 MHz, $\mathrm{CDCl}_{3}$ )

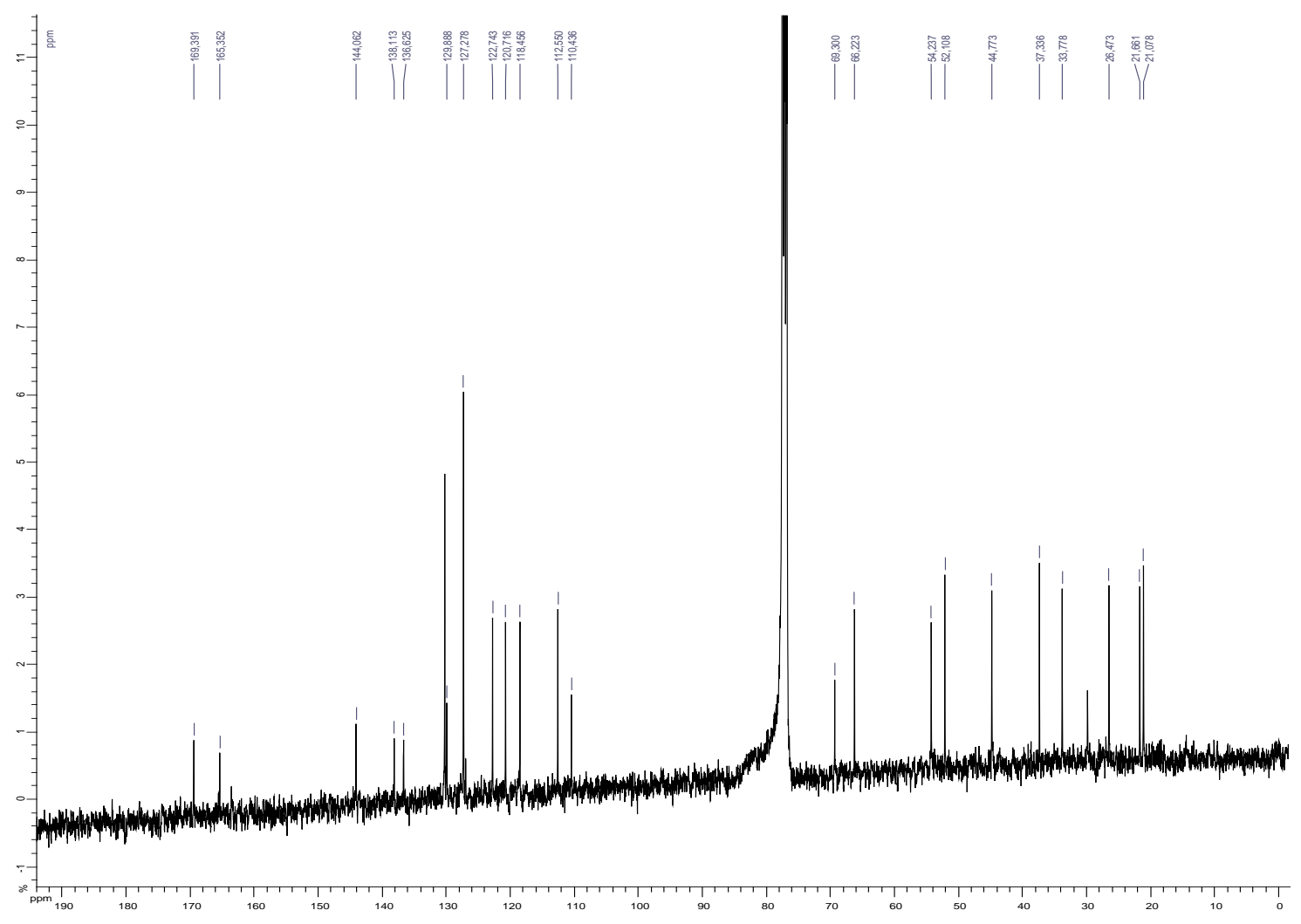


${ }^{1} \mathrm{H}$ NMR $\left(300 \mathrm{MHz}, \mathrm{CDCl}_{3}\right)$

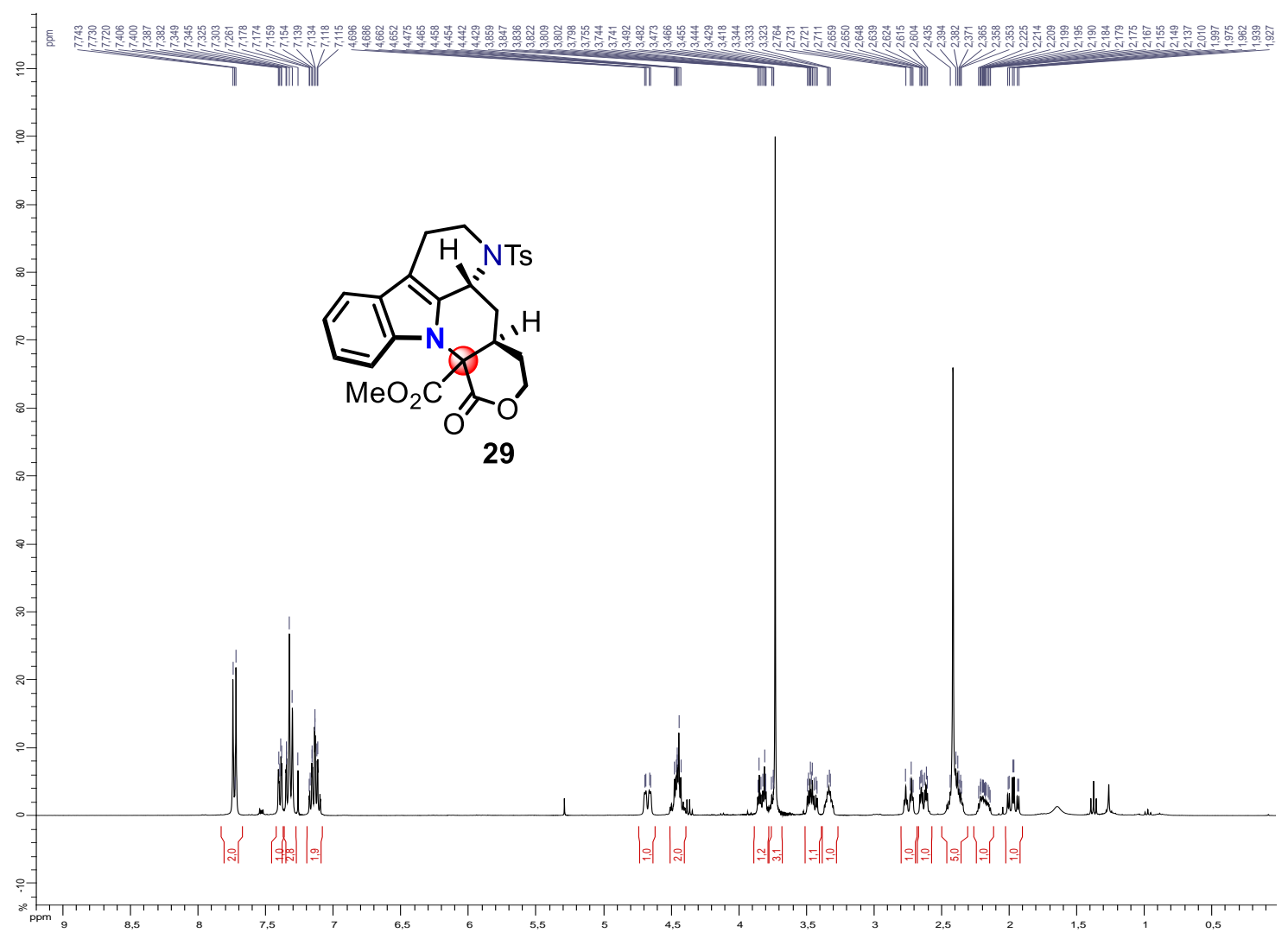

${ }^{13} \mathrm{C}$ NMR (91 MHz, $\mathrm{CDCl}_{3}$ )

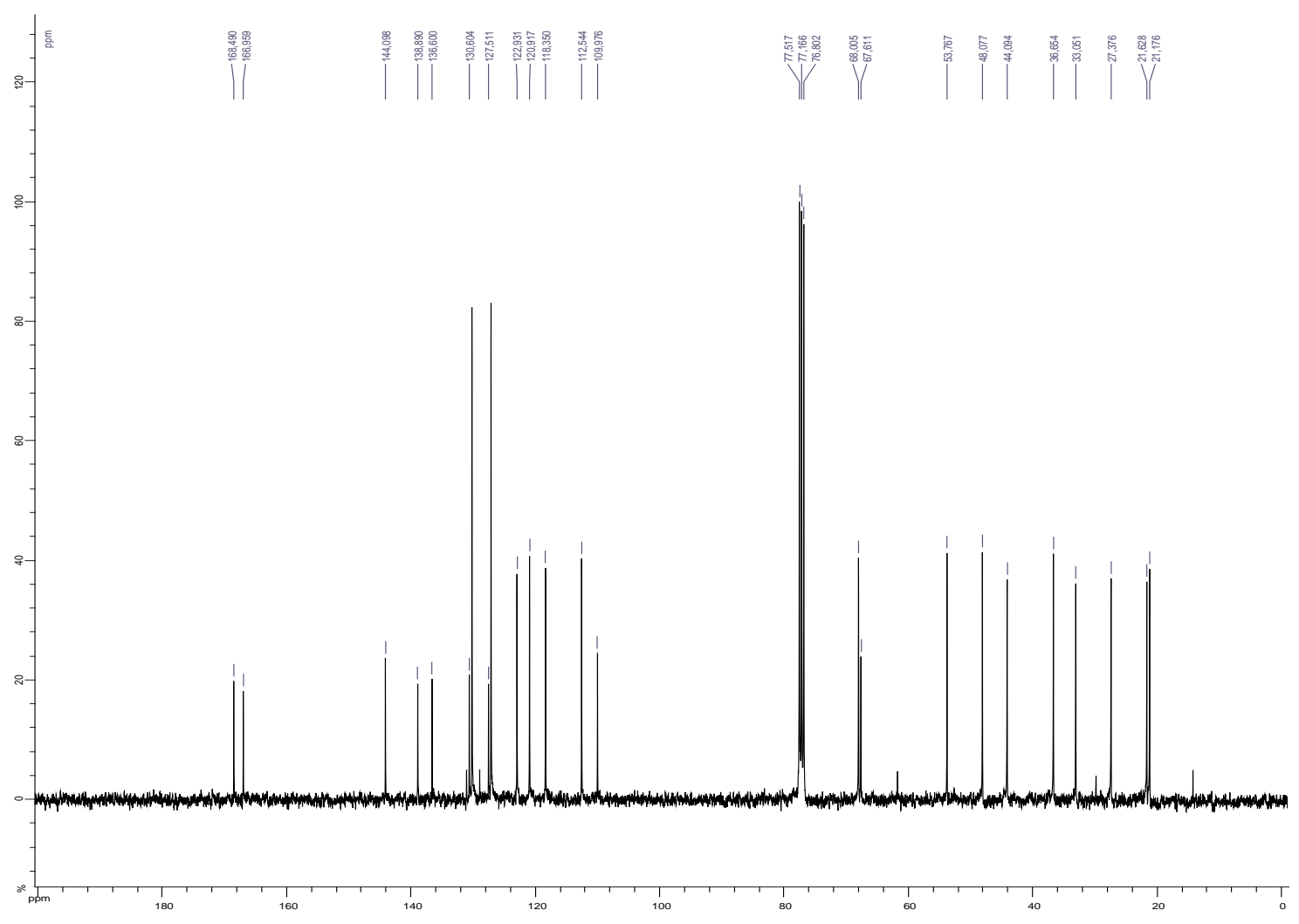




\section{HPLC chromatograms of $17 a$ and $25 a$}

\section{HPLC chromatograms of racemic (top) and enantioenriched 17a (bottom)}

Operator:hplc Timebase:U_3000 Sequence:Guillaume Vincent HPLC Page 1-1
6/1/2020 2:01 PM

\begin{tabular}{|llll|}
\hline 315 MJ1140-1 & & \\
& & \\
\hline Sample Name: & MJ1140-1 & Injection Volume: & 6,0 \\
Vial Number: & RB7 & Channel: & UV_VIS_1 \\
Sample Type: & unknown & Wavelength: & 222 \\
Control Program: & ADH9505temp25 & Bandwidth: & 10 \\
Quantif. Method: & Methode generique & Dilution Factor: & 1,0000 \\
Recording Time: & $28 / 11 / 201911: 36$ & Sample Weight: & 1,0000 \\
Run Time (min): & 90,00 & Sample Amount: & 1,0000 \\
\hline
\end{tabular}

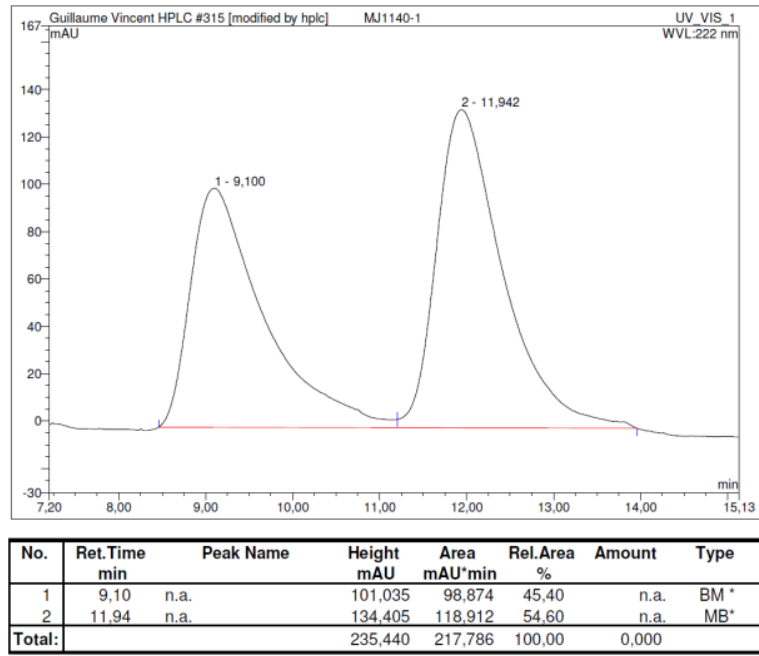

Operator:hplc Timebase:U 3000 Sequence:Guillaume Vincent HPLC Page 1-
$6 / 1 / 2020$ 2:03 PM

\begin{tabular}{|llll|}
\hline 318 MJ1141-1 & & \\
& & \\
\hline Sample Name: & MJ1141-1 & Injection Volume: & 6,0 \\
Vial Number: & RD7 & Channel: & UV_VIS_1 \\
Sample Type: & unknown & Wavelength: & $\mathbf{2 2 2}$ \\
Control Program: & ADH9505temp25 & Bandwidth: & 10 \\
Quantif. Method: & Methode generique & Dilution Factor: & $\mathbf{1 , 0 0 0 0}$ \\
Recording Time: & $28 / 11 / 2019$ 13:39 & Sample Weight: & $\mathbf{1 , 0 0 0 0}$ \\
Run Time (min): & 90,01 & Sample Amount: & $\mathbf{1 , 0 0 0 0}$ \\
\hline
\end{tabular}

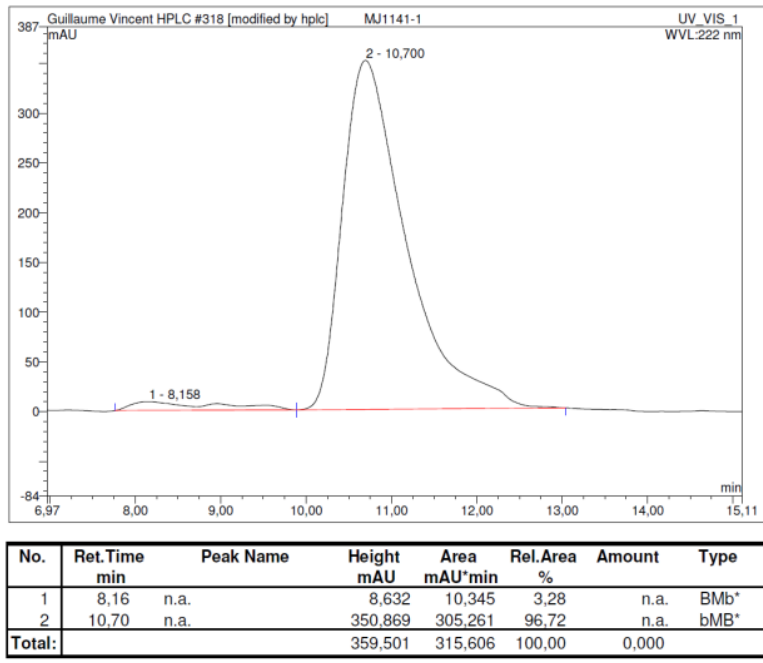


HPLC chromatograms of racemic (top) and enantioenriched 25a (bottom)

Operator:hplc Timebase:U 3000 Sequence:Guillaume Vincent HPLC Page 1-1
6/1/2020 1:47 PM

\begin{tabular}{|llll|}
\hline 344 MJ1140-2 & & \\
& & \\
\hline Sample Name: & MJ1140-2 & Injection Volume: & 6,0 \\
Vial Number: & RA7 & Channel: & UV_VIS_1 \\
Sample Type: & unknown & Wavelength: & 240 \\
Control Program: & ADH9505temp05 & Bandwidth: & 10 \\
Quantif. Method: & Methode generique & Dilution Factor: & 1,0000 \\
Recording Time: & $3 / 12 / 2019$ 17:10 & Sample Weight: & 1,0000 \\
Run Time (min): & 60,00 & Sample Amount: & 1,0000 \\
\hline
\end{tabular}

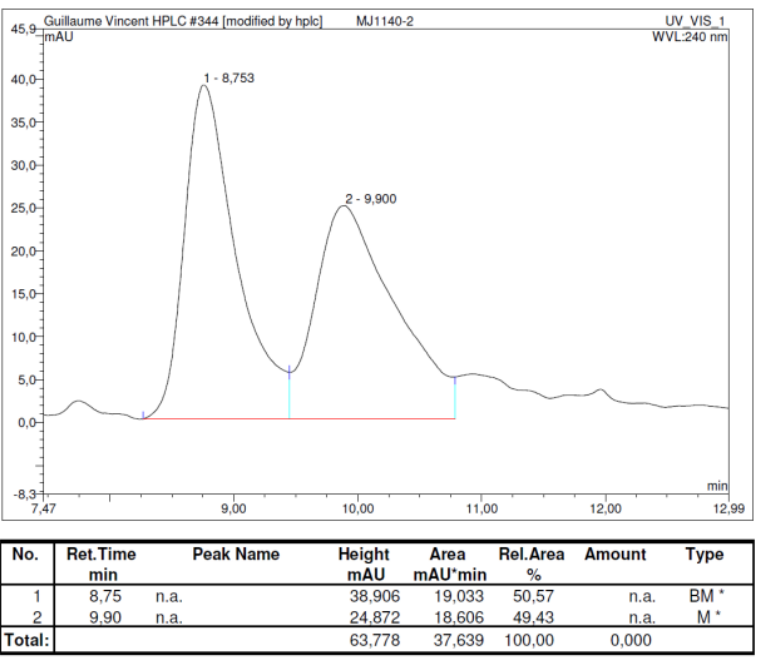

Operator:hplc Timebase:U 3000 Sequence:Guillaume Vincent HPLC Page
$6 / 1 / 2020$ 1:44

\begin{tabular}{|llll|}
\hline 347 MJ1141-2 & & \\
& & & \\
\hline Sample Name: & MJ1141-2 & Injection Volume: & 6,0 \\
Vial Number: & RC7 & Channel: & UV_VIS_1 \\
Sample Type: & unknown & Wavelength: & 240 \\
Control Program: & ADH9505temp05 & Bandwidth: & 10 \\
Quantif. Method: & Methode generique & Dilution Factor: & $\mathbf{1 , 0 0 0 0}$ \\
Recording Time: & $3 / 12 / 201918: 43$ & Sample Weight: & 1,0000 \\
Run Time (min): & 60,00 & Sample Amount: & $\mathbf{1 , 0 0 0 0}$ \\
\hline
\end{tabular}

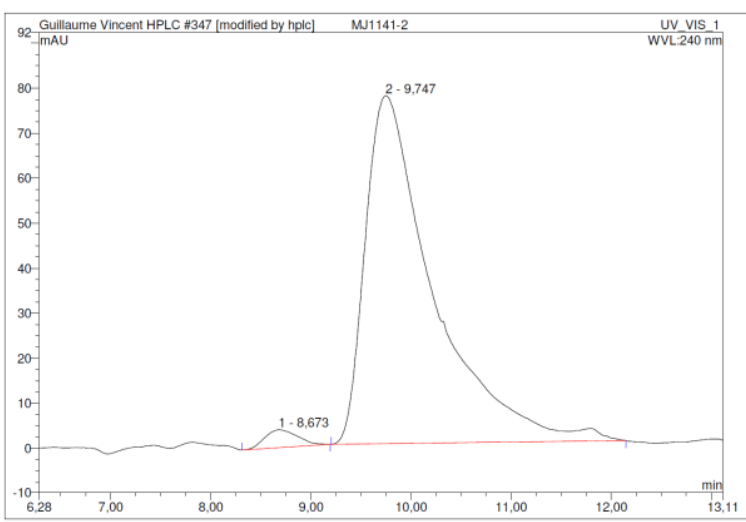

\begin{tabular}{|c|c|c|c|c|c|c|c|}
\hline No. & $\begin{array}{c}\text { Ret.Time } \\
\min \end{array}$ & Peak Name & $\begin{array}{c}\text { Height } \\
\text { mAU }\end{array}$ & $\begin{array}{c}\text { Area } \\
\text { mAU*min }\end{array}$ & $\begin{array}{c}\text { Rel.Area } \\
\%\end{array}$ & Amount & Type \\
\hline 1 & 8,67 & n.a. & 3,997 & 1,508 & 2,42 & n.a. & $\mathrm{BMB}^{\prime}$ \\
\hline 2 & 9,75 & n.a. & 77,270 & 60,829 & 97,58 & n.a. & BMB' \\
\hline Total: & & & 81,268 & 62,337 & 100,00 & 0,000 & \\
\hline
\end{tabular}




\section{References}

1. Zhang, W.; Bah, J.; Wohlfarth, A.; Franzén, J., Chem. - Eur. J. 2011, 17, 13814-13824.

2. Zhang, H.; Ma, X.; Kang, H.; Hong, L.; Wang, R., Chem. - Asian J. 2013, 8, 542-545.

3. Rodrigo, E.; García Ruano, J. L.; Cid, M. B., J. Org. Chem., 2013, 78, 10737-10746. 